Portland State University

PDXScholar

Summer 9-26-2017

\title{
Uncovering Coxiella burnetii's Pathogenicity by Elucidating its Metabolism and Host Interactions
}

Jess Annai Millar

Portland State University

Follow this and additional works at: https://pdxscholar.library.pdx.edu/open_access_etds

Part of the Microbiology Commons

Let us know how access to this document benefits you.

\section{Recommended Citation}

Millar, Jess Annai, "Uncovering Coxiella burnetii's Pathogenicity by Elucidating its Metabolism and Host Interactions" (2017). Dissertations and Theses. Paper 3937.

https://doi.org/10.15760/etd.5821

This Thesis is brought to you for free and open access. It has been accepted for inclusion in Dissertations and Theses by an authorized administrator of PDXScholar. Please contact us if we can make this document more accessible: pdxscholar@pdx.edu. 
Uncovering Coxiella burnetii's Pathogenicity by Elucidating its

Metabolism and Host Interactions

by

Jess Annai Millar

A thesis submitted in partial fulfillment of the

requirements for the degree of

\author{
Master of Science \\ in \\ Biology \\ Thesis Committee: \\ Rahul Raghavan \\ Susan E. Masta \\ Kenneth M. Stedman
}

Portland State University

2017 


\begin{abstract}
Coxiella burnetii, the etiologic agent of acute Q fever and chronic endocarditis, has a unique biphasic life cycle, which includes a metabolically active intracellular form that occupies a large lysosome-derived acidic vacuole. C. burnetii is the only bacterium known to thrive within such a hostile intracellular niche, and this ability is fundamental to its pathogenicity; however, very little is known about genes that facilitate Coxiella's intracellular growth. This lack of knowledge of Coxiella's basic biology and molecular pathogenesis is a critical barrier to developing more effective therapies.

In this study, we aimed to understand both bacterial and host factors that have important roles during C. burnetii infections. Using an evolutionary genomics approach, we identified metabolic pathways that are critical to $C$. burnetii's ability to grow intracellularly. Among those found, the most promising are fatty acid, biotin, and heme biosyntheses pathways. Coxiella has horizontally acquired extra copies of genes that enhance these processes; when these genes were disrupted, Coxiella's growth was significantly inhibited. Also, by analyzing the host transcriptome, we identified human genes, including microRNA (miRNA) genes that are important during C. burnetii infections. Coxiella induces the expression of multiple anti-apoptotic miRNAs, which likely have a role in inhibiting apoptosis in order to sustain the intracellular replication of the pathogen. The biosynthetic pathways and miRNAs identified in this study are ideal targets for developing more effective therapeutic strategies against $\mathrm{Q}$ fever and its chronic and often fatal complications.
\end{abstract}




\section{ACKNOWLEDGEMENTS}

I would like to thank my research advisor Rahul Raghavan of the Biology Department at Portland State University. He has provided continual support but also generous freedom that has allowed me to design aspects and conduct much of this project independently. I would also like to acknowledge Rahul and Tina Schroyer for their assistance in writing my thesis. Their feedback has helped me become a better writer. I want to thank Abraham Moses and Fenil Kacharia for their assistance with conducting all the physical experiments and other lab duties. I also want to thank Todd Smith for taking the time to supplement my learning in bioinformatics. I would also like to thank my thesis committee: Susan Masta and Kenneth Stedman. I appreciate their time and effort reviewing my paper and supporting my research and education.

This work was supported by startup funds from PSU to Rahul Raghavan, as well as the American Heart Association Beginning Grant-in-Aid, Collins Medical Trust Research Grant, Medical Research Foundation of Oregon New Investigator Grant, and NIH National Institute of Allergy and Infectious Diseases grants (R03 AI123464-01A1, R15 AI126385-01A1). Fellowship and partial funding was provided by the Portland State Laurels Graduate Award, Elsa Jorgenson Award, President's Equal Access Scholarship, and Forbes-Lea Research Award at PSU. Outside funding was also provided by the Sigma Xi Grants-in-Aid of Research Copernicus Fund, as well as travel funds from American Society for Microbiology, Pacific Northwest Women in Science, Society for Applied Microbiology, and Society for the Study of Evolution. 


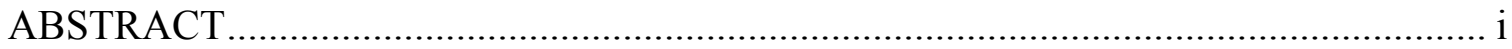

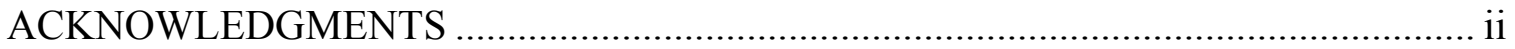

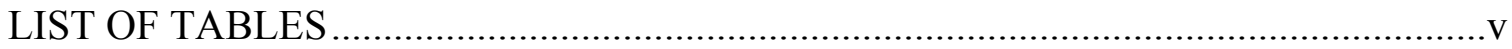

LIST OF FIGURES ....................................................................................... vi

\section{CHAPTERS}

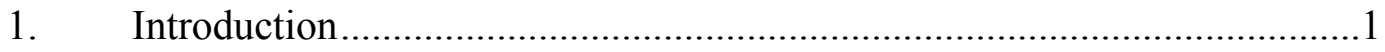

2. Horizontally acquired biosynthesis genes boost Coxiella burnetii's

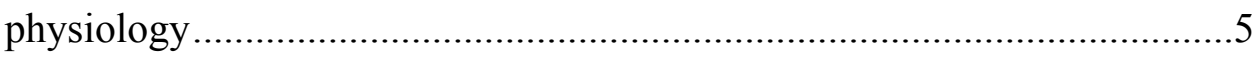

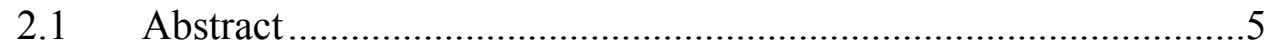

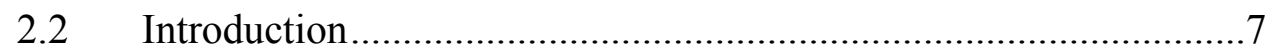

2.3 Materials and Methods..................................................................10

$2.4 \quad$ Results and Discussion ..............................................................13

3. Coxiella burnetii and Leishmania mexicana residing within similar parasitophorous vacuoles elicit disparate host responses ............................30

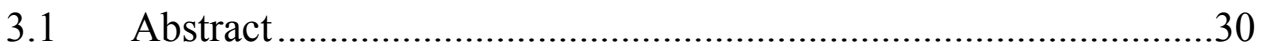

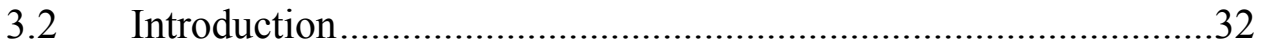

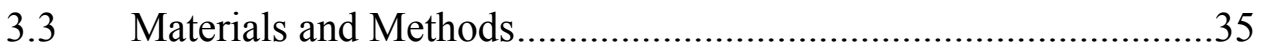

3.4 Results and Discussion .................................................................

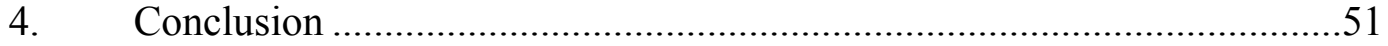




\section{APPENDICES}

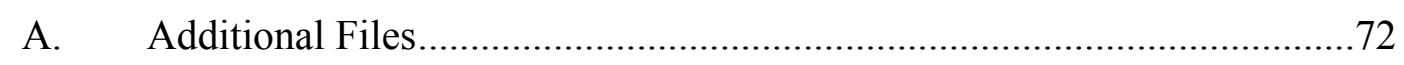

B. Genome rearrangements can make and break small RNA genes ..............75

C. Emergence of new sRNAs in enteric bacteria is associated with low

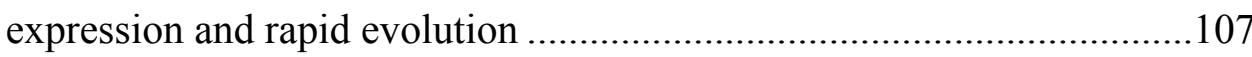

D. Accumulation and expression of multiple antibiotic resistance genes in Arcobacter cryaerophilus that thrives in sewage..................................142

E. Whole-genome sequence of Coxiella burnetii Nine Mile RSA 439 (phase

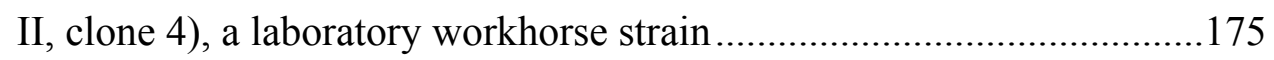




\section{LIST OF TABLES}

PAGE

\section{TABLES}

2.1 Expression of biotin and heme biosyntheses genes in C. burnetii...........24

3.1 KEGG pathways enriched in Coxiella-infected and Leishmania-infected

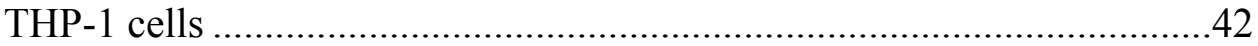

3.2 MicroRNAs perturbed by Coxiella and Leishmania infections.................49 


\section{LIST OF FIGURES}

PAGE

\section{FIGURES}

1.1 Biogenesis of Coxiella-containing vacuole (CCV).................................2

2.1 Horizontally acquired genes in C. burnetii ..........................................15

2.2 Maximum Likelihood tree of CBU_0678, gained via HGT ......................18

2.3 Bayesian tree of CBU_0678, gained via HGT.....................................19

2.4 A horizontally acquired fatty acid biosynthesis operon in C. burnetii ......21

2.5 Bayesian tree of CBU_0038, gained via HGT.......................................22

2.6 Heme and biotin syntheses effect on C. burnetii's growth ......................26

2.7 $\mathrm{tRNA}^{\mathrm{Glu}} 2$ was horizontally acquired by $C$. burnetii ..............................27

3.1 Identification of differentially expressed genes ...............................40

3.2 Protein-protein interaction analysis ................................................44

3.3 Validation by qPCR of expression levels estimated by RNA-seq............45

3.4 Differential expression of mRNA isoforms .....................................47 


\section{CHAPTER 1}

Introduction

Coxiella burnetii is the etiological agent of acute Q fever and a chronic disease commonly manifested as endocarditis (Maurin and Raoult 1999). C. burnetii has a unique biphasic lifestyle. The pathogen persists in the environment as a metabolically quiescent small cell variant (SCV), which transforms into a metabolically active large cell variant (LCV) within an acidic ( $\mathrm{pH} \sim 4.5$ ) lysosome-derived Coxiella-containing vacuole (CCV) (Figure 1.1; Voth and Heinzen 2007). Coxiella is the only bacterium known to thrive in such a compartment, and this ability is considered critical to its pathogenicity.

CDC has classified Coxiella as a select agent due to its past use as a bioweapon, environmental stability, aerosol transmission, and extremely low infectious dose [ID50 of one to ten bacteria in a guinea pig model (Tigertt et al. 1961)]. Untreated chronic Q fever is associated with high mortality rate ( $>60 \%$ ) (Karakousis et al. 2006) and is very difficult to treat because it requires prolonged antibiotic regimen: a combination of doxycycline and hydroxychloroquine (1.5 to 3 years), or doxycycline and fluoroquinolone (3 to 4 years) (Gould et al. 2012). However, strains resistant to doxycycline are prevalent (Rouli et al. 2012). C. burnetii is found worldwide and can cause epidemics, such as a recent epidemic in the Netherlands where thousands of people were infected (van der Hoek et al. 2012). It has also been detected among U.S. military personnel (Faix et al. 2008), and its high prevalence in US environmental samples (Kersh et al. 2010; Loftis et al. 2010) highlights the urgent need to understand its biology and 


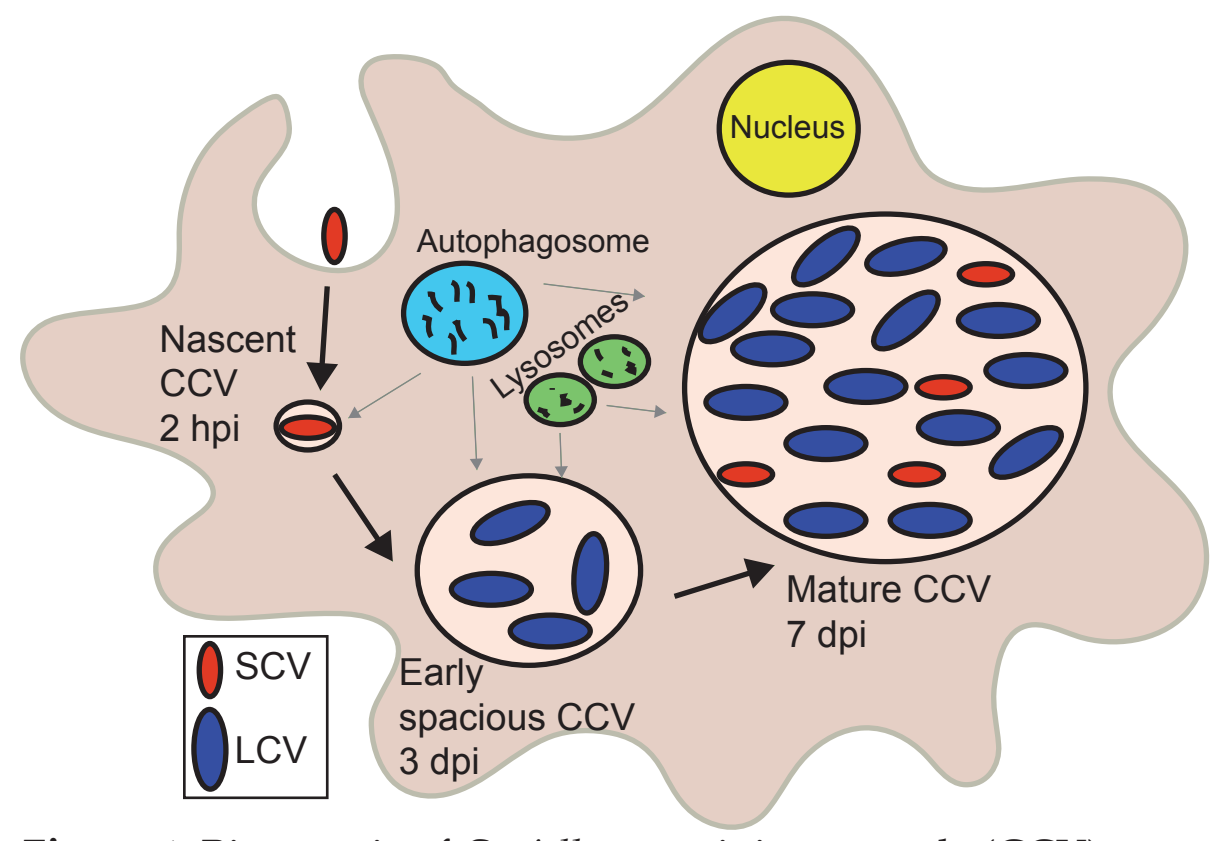

Figure 1.1 - Biogenesis of Coxiella-containing vacuole (CCV). The pathogen occurs as a 'spore-like' small cell variant (SCV) in the environment. Most human infections occur through inhalation of aerosols contaminated with animal material. Shortly after entering into a host cell (typically an alveolar macrophage), SCV transforms into a metabolically active form called the large cell variant (LCV) within a lysosome-derived, acidic $(\mathrm{pH}$ 4.5) Coxiella-containing vacuole (CCV). 
virulence mechanisms.

Current research into Coxiella's pathogenicity is mainly focused on host cell entry and on manipulation of the host cell environment through its Type Four Secretion System (TFSS) (e.g., Weber et al. 2013; Newton et al. 2014; Larson et al. 2015, Graham et al. 2015; Seshadri et al. 2003; Beare et al. 2009, Chen et al. 2010). This bias has left a large void in our understanding of Coxiella's intracellular metabolism and host response to Coxiella infection. This lack of knowledge of Coxiella's basic biology and molecular pathogenesis is a critical barrier to developing more effective therapies.

In this study, we aimed to understand both bacterial and host factors that have important roles during C. burnetii infections. In Chapter 2, using an evolutionary genomics approach, we identified metabolic pathways that are critical to C. burnetii's ability to grow intracellularly. Among those found, the most promising are fatty acid, biotin, and heme biosyntheses pathways. Coxiella has horizontally acquired extra copies of genes that enhance these processes; when these genes are disrupted Coxiella's growth in the cell is significantly inhibited. In Chapter 3, by analyzing the host transcriptome, we identified host genes, including microRNA (miRNA) genes that are important during $C$. burnetii infections. Coxiella induces the expression of multiple anti-apoptotic miRNAs, which likely have a role in inhibiting apoptosis in order to sustain the intracellular replication of the pathogen. One well-studied example is miR-148a, which inhibits apoptosis by targeting BCL-2, thereby possibly promoting Coxiella infection. The biosynthetic pathways and miRNAs identified in this study are ideal targets for 
developing more effective therapeutic strategies against $\mathrm{Q}$ fever and its chronic and often fatal complications. 


\section{CHAPTER 2}

Horizontally acquired biosynthesis genes boost Coxiella burnetii's physiology

\subsection{ABSTRACT}

Coxiella burnetii, the etiologic agent of acute $\mathrm{Q}$ fever and chronic endocarditis, has a unique biphasic life cycle, which includes a metabolically active intracellular form that occupies a large lysosome-derived acidic vacuole. C. burnetii is the only bacterium known to thrive within such an hostile intracellular niche, and this ability is fundamental to its pathogenicity; however, very little is known about genes that facilitate Coxiella's intracellular growth. Recent studies indicate that $C$. burnetii evolved from a tickassociated ancestor and that the metabolic capabilities of $C$. burnetii are different from that of Coxiella-like bacteria found in ticks. Horizontally acquired genes that allow $C$. burnetii to infect and grow within mammalian cells likely facilitated the host shift; however, because of its obligate intracellular replication, C. burnetii would have lost most genes that have been rendered redundant due to the availability of metabolites within the host cell. Based on these observations, we reasoned that horizontally derived biosynthetic genes that have been retained in the reduced genome of $C$. burnetii are ideal candidates to begin to uncover its intracellular metabolic requirements. Our analyses identified a large number of putative genes of foreign-origin in C. burnetii, including $\mathrm{tRNA}^{\mathrm{Glu}} 2$ that is potentially required for heme biosynthesis, and genes involved in the production of lipopolysaccharide - a virulence factor, and of critical metabolites such as 
fatty acids and biotin. In comparison to wild-type C. burnetii, a strain that lacks tRNA ${ }^{\mathrm{Glu}_{2}}$ exhibited reduced growth, indicating its importance to Coxiella's physiology.

Additionally, by using chemical agents that block heme and biotin biosyntheses, we show that these pathways are promising targets for the development of new anti-Coxiella therapies. 


\subsection{INTRODUCTION}

Coxiella burnetii is the etiological agent of acute $\mathrm{Q}$ fever and a chronic disease commonly manifested as endocarditis (Maurin and Raoult 1999). Most human infections occur through inhalation of aerosols originating from ruminants that shed C. burnetii during parturition and in milk. The pathogen persists in the environment as a metabolically quiescent small cell variant (SCV), which transforms into a metabolically active large cell variant (LCV) within a lysosome-derived, acidic ( $\mathrm{pH} \sim 4.5)$, Coxiellacontaining vacuole (CCV) (Voth and Heinzen 2007). The unique ability of $C$. burnetii to thrive in this inhospitable vacuole is fundamental to its physiology and pathogenicity; however, the metabolic processes that allow its intracellular growth are unknown.

The evolutionary origin of $C$. burnetii is also not clearly understood. The closest relatives of $C$. burnetii are tick-associated bacteria, indicating that $C$. burnetii evolved from a tick-associated ancestor (Smith et al. 2015; Duron et al. 2015; Gottlieb et al. 2015). Interestingly, Coxiella-like bacteria found in ticks cannot infect mammalian cells and are unable to grow in ACCM-2, a culture medium that supports robust growth of $C$. burnetii (Duron et al. 2015; Omsland et al. 2011). These observations suggest that despite their close evolutionary relationship, the human pathogen and the tick-associated strains have different virulence and metabolic capabilities. Concomitantly, when the genomes of C. burnetii were sequenced, it became clear that the pathogen has acquired several virulence and metabolic genes via horizontal gene transfer (HGT) (Raghavan et al. 2008). For example, C. burnetii contains a tryptophan biosynthesis operon of Chlamydial origin, 
and a Type Four Secretion System (TFSS) and eukaryote-like ankyrin repeat sequencecontaining effector proteins that are essential for CCV generation (Seshadri et al. 2003; Beare et al. 2009). Horizontal acquisition of foreign DNA occurs in bacteria through transformation, conjugation or transduction via mobile genetic elements such as plasmids, integrons, bacteriophages, transposons, retrotransposons etc (Ochman et al. 2000). The novel DNA is stably maintained and spreads through the recipient population if it offers selective advantage (e.g. antibiotic resistance), allowing the bacterium to adapt to the new environment (Eisen 2000; Frost et al. 2005; Thomas and Nielsen 2005).

Unlike eukaryotic genomes, which contain large fractions of nonfunctional DNA (e.g., $>80 \%$ of human genome), bacterial genomes are tightly packed with functional genes (Moran 2002; Ochman and Davalos 2006). In bacteria there is a bias towards deletion over insertion, hence, DNA is retained in a bacterial genome only if selection is acting effectively to preserve it (Mira et al. 2001). For instance, when the tick-associated ancestor of C. burnetii evolved into a mammalian pathogen that replicates only within the $\mathrm{CCV}$, several biosynthetic genes would be rendered redundant if corresponding metabolic intermediates are available within the host cell. In addition, although genes acquired via HGT might have been critical in facilitating the host shift, many of them could become expendable in the new lifestyle (e.g. Lo et al. 2015). These superfluous metabolic and HGT-origin genes would subsequently be deleted from the genome due to a lack of selection pressure to maintain them (Ochman and Moran 2001). Furthermore, the intracellular niche limits C. burnetii's opportunity to gain new genes from the environment via HGT. Thus, intracellular pathogens such as $C$. burnetii tend to have 
reduced genomes in comparison to related free-living bacteria (e.g., C. burnetii's genome is $\sim 2$ million bp, whereas $E$. coli's is $\sim 5$ million bp). Based on these observations, we reasoned that a significant number of HGT-origin genes that have been retained in $C$. burnetii would be critical to its intracellular fitness. In this study, we identified a large number of horizontally derived genes, including those for the synthesis of LPS, fatty acids, heme, and biotin that augment the physiological capability of $C$. burnetii. 


\subsection{MATERIALS AND METHODS}

\section{C. burnetii growth assay}

C. burnetii Nine Mile phase II RSA 439 was grown in ACCM-2 medium as described previously (Omsland et al. 2011; Warrier et al. 2014), and incubated at $37^{\circ} \mathrm{C}, 2.5 \% \mathrm{O}_{2}$, and 5.0\% $\mathrm{CO}_{2}$ using a Galaxy $170 \mathrm{R}$ incubator (New Brunswick Scientific, NJ).

Chloramphenicol $(8 \mu \mathrm{g} / \mathrm{ml})$ and/or Kanamycin $(375 \mu \mathrm{g} / \mathrm{ml})$ were added as necessary. Growth was measured using PicoGreen as described previously (Martinez et al. 2015). Briefly, $50 \mu 1$ of culture was mixed with $5 \mu 1$ of Triton X-100 Surfact-Amps $10 \%$ detergent solution (Thermo Scientific) in 96-well black-bottom Cliniplates (Thermo Scientific), and allowed to incubate at room temperature for 10 minutes with shaking. PicoGreen (Life Technologies) was diluted 1:200 in TE buffer and 55 $\mu \mathrm{L}$ was added to the wells, and incubated at room temperature with shaking for 5 minutes. Wells were excited at $495 \mathrm{~nm}$ and emission was read at $519 \mathrm{~nm}$ using a Victor X5 2030 Multiplate Reader (Perkin Elmer). To determine whether Coxiella can utilize external heme as its sole iron source, ferrous sulfate was either omitted from ACCM-2 preparations or substituted with hemin (Alfa Aesar), and to assay the importance of heme and biotin biosyntheses, gabaculine (Enzo Life Sciences) or MAC13772 (Maybridge) was added to ACCM-2. Briefly, a $10 \mathrm{mM}$ stock of hemin was made in $1.5 \mathrm{M} \mathrm{NaOH}$, a $300 \mathrm{mg} / \mathrm{mL}$ stock of MAC13772 was made in dimethyl sulfoxide (DMSO), and a $100 \mathrm{mM}$ stock of gabaculine was made in distilled water. One $\mu$ l of solution from these stocks was added to $1 \mathrm{ml}$ of ACCM-2 to attain final concentrations of $10 \mu \mathrm{M}$ hemin, $300 \mu \mathrm{g} / \mathrm{ml} \mathrm{MAC} 13772$, 
or $100 \mu \mathrm{M}$ gabaculine. ACCM-2 containing the same amount of solvent was used as control, and growth was measured after 7 days using PicoGreen as described above.

\section{Generation of tRNA ${ }^{\text {Glu }} 2$ deletion and complementation strains}

To delete tRNA ${ }^{\text {Glu }} 2, \sim 1200$ bp on each side of the gene along with Chloramphenicol acetyltransferase (CAT) gene was cloned into the vector pJC-Kan, and the mutant was generated as described previously (Beare et al. 2012). Insertion of the CAT gene in place of tRNA ${ }^{\text {Glu }} 2$ was confirmed using PCR and DNA sequencing. To generate a complementation strain, we used pKM244, a low-copy plasmid that is maintained stably

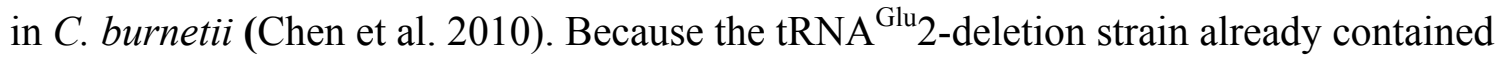
a CAT gene, we amplified a kanamycin resistance gene under the control of the 1169 promoter from pJB-Kan (Beare et al. 2012) and cloned it into pKM244 using NheI and AatII to generate pAM100. We inserted tRNA ${ }^{\text {Glu }} 2$ along with its flanking intergenic regions into pAM100 using BamHI. C. burnetii was transformed (400 ohms, $2.5 \mathrm{kV}, 25$

$\mathrm{mF}$ ) with either empty pAM100 or pAM100 with cloned tRNA ${ }^{\mathrm{Glu}} 2$, as described previously (Omsland et al. 2011; Beare et al. 2012). Growth in ACCM-2 of wild-type (with empty pAM100), deletion (with empty pAM100) and complementation strains were measured at day-7 using PicoGreen as described above.

\section{Detection of horizontal gene transfer}

Horizontally acquired genes were identified using HGTector (Zhu et al. 2014). Coxiella was set as self-group, and Legionellales was set as exclusion group. BLAST parameter 
thresholds were set at $70 \%$ identity and an E-value of a maximum of $1 \mathrm{e}-5$. To validate the HGT data, phylogenetic analyses were conducted for several genes projected to be the result of HGT. To this end, TBLASTN search was conducted against the NCBI nr database and the top 100 matches with at least $30 \%$ identity, $70 \%$ coverage, and E-value of less than 1e-10 were chosen. Sequence alignment was performed using Clustal Omega (Sievers et al. 2011), and ambiguously aligned regions were removed using Gblocks (Talavera and Castresana 2007). The evolution model GTR $+\mathrm{I}+\mathrm{G}$ (General Time Reversible plus Invariant sites plus Gamma distribution) was selected using jModelTest2 (Darriba et al. 2012). Bayesian trees were constructed using MrBayes as implemented in Geneious (Huelsenbeck and Ronquist 2001; Kearse et al. 2012). A chain length of $1,000,000$ was used with a burn-in fraction of $25 \%$ and sampling every 100 trees. Maximum Likelihood trees were constructed using RAxML (Stamatakis et al. 2008) as implemented in Geneious with 1,000 bootstrap replicates. 


\subsection{RESULTS AND DISCUSSION}

\section{Identification of horizontally acquired genes in $C$. burnetii}

HGT is a major driver of evolution and adaptation in bacteria (Lerat et al. 2005; Price et al. 2008; Treangen and Rocha 2011). By examining differences in nucleotide composition (e.g. GC\%) HGT-origin genes can be tentatively identified; however, because most transfers occur between closely related bacteria, and because genes from distant organisms will evolve over time to reflect the base composition of the recipient genome (Lawrence and Ochman 1997), this approach is not always effective. Another common approach is to perform reciprocal-BLAST analysis (e.g. Raghavan et al. 2012), where, if the top hit is from a distantly related organism, the gene is of putative HGT origin. While this approach provides rapid results, the best BLAST hit may not always be the closest phylogenetic relative of the query gene (Koski and Golding 2001). This is especially the case for organisms such as $C$. burnetii that have few close relatives represented in public NCBI databases. For instance, C. burnetii is the only defined species within its genus, and complete genomes are available for only three other bacteria within the order Legionellales — Legionella, Rickettsiella, and Diplorickettsia. In order to circumvent this limitation, we utilized a phylogeny-informed BLAST-based approach (HGTector, Zhu et al. 2014), but applied very strict criteria to categorize a gene as horizontally acquired. We set Coxiella as the self-group, and Legionellales as the exclusion group, which captured HGT events where only Coxiella has acquired a particular gene from outside of Legionellales and ignored any events where the genes 
could also have been transferred elsewhere within the order. In addition, because the phylogenetic position of Legionellales within Gammaproteobacteria is not well resolved (Williams et al. 2010), we also ignored any gene that was potentially gained from another member of the class Gammaproteobacteria. Using this ultra-conservative approach, we were able to identify 172 'high-confidence' horizontally acquired genes on the chromosome of C. burnetii RSA 493 (Figure 2.1, Additional_file_A1.csv), whereas, none of the genes located on C. burnetii plasmids (QpH1, QpRS, QpDG) were deemed to be of horizontal origin.

Orthologs of all 172 HGT-origin genes are present in C. burnetii Dugway 5J108111 (NC_009727.1), C. burnetii 3262 (CP013667.1) and C. burnetii Z3055 (NZ_LK937696.1), however orthologs of CBU_1991 were not detected in C. burnetii RSA 331 (CP000890.1), CBU_2021 and CBU_0562 in C. burnetii G_Q212 (NC_011527.1), and CBU_0007a, CBU_0167, CBU_0168, CBU_0768 and CBU_0792 in C. burnetii K_Q154 (NC_011528.1). Of the 172 HGT genes, 18 are located close to transposase genes in C. burnetii RSA 493, suggesting a role for transposons or insertion sequences in acquiring foreign genes. In addition to the large number of transposases (>30), all C. burnetii strains contain several other selfish genetic elements that are horizontally exchanged between bacteria, including two Group I introns, an intein, and an intervening sequence (Seshadri et al. 2003; Beare et al. 2009; Raghavan et al. 2007; Raghavan et al. 2008; Warrier et al. 2016). Proliferation of mobile genetic elements and extensive genome rearrangements are hallmarks of bacteria that have recently shifted to host- associated lifestyles, indicating that the obligate intracellular growth of $C$. burnetii 


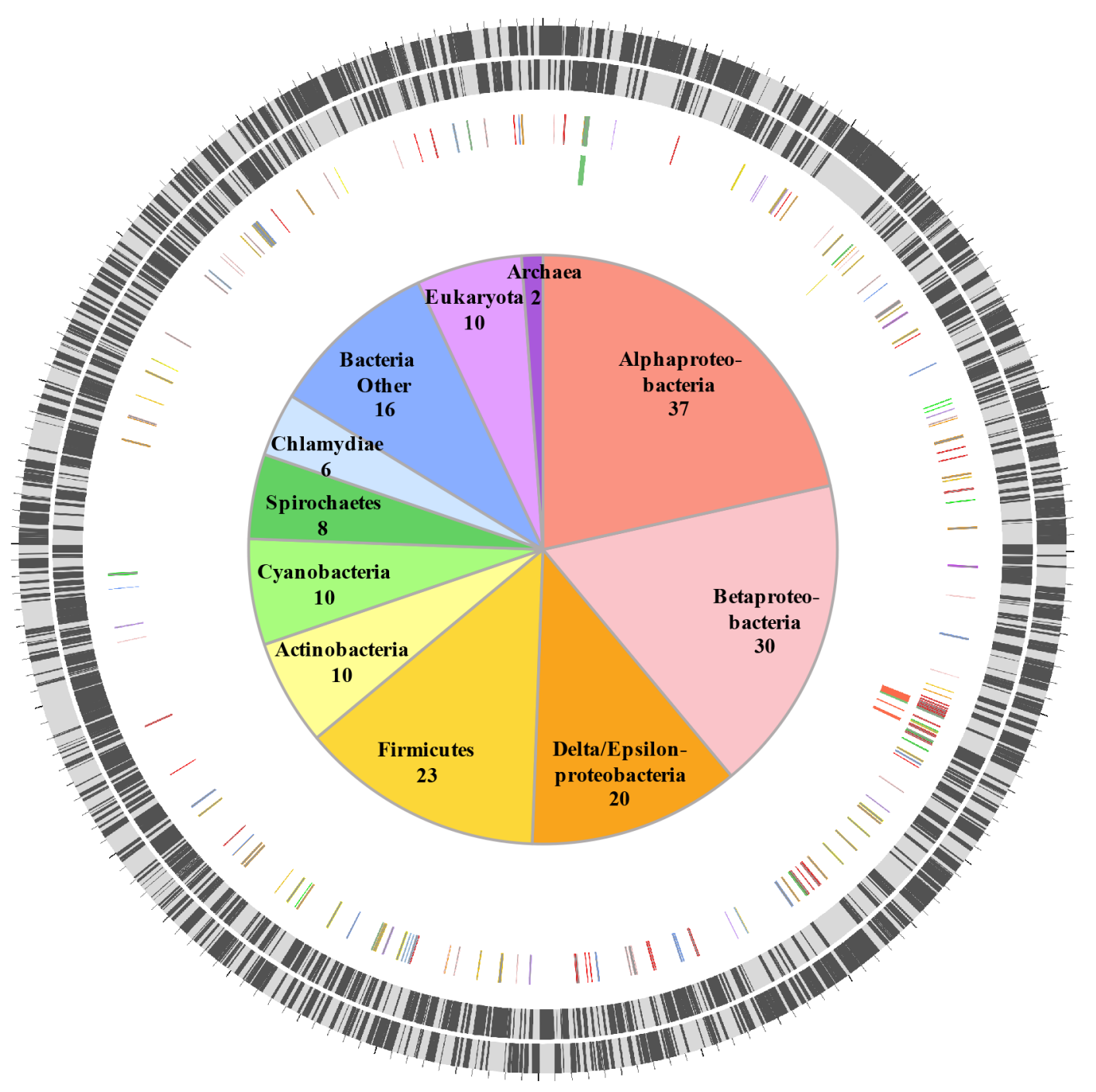

Figure 2.1 - Horizontally acquired genes in C. burnetii. Two outer rings show ORFs (black bars) on forward and reverse strands, respectively. Third ring shows positions of 172 HGT-origin genes, and the inner ring contains biosynthetic genes examined in this study. Genes are colored according to their putative donors, as shown in the center. Number of genes acquired from each donor is also shown. 
is of recent origin (Ochman and Moran 2001; McCutcheon and Moran 2012).

As expected, our analysis excluded several genes that were likely acquired via HGT because they are also present in other members of the order Legionellales e.g. TFSS genes, eukaryote-like sterol reductase genes, plasmid genes with eukaryotic domains etc. (Seshadri et al. 2003; Beare et al. 2009; Gilk et al. 2010; Voth et al. 2013). Without better resolution of $C$. burnetii's phylogenetic position in the bacterial tree, it is difficult to discern whether these genes were acquired from a common ancestor or were gained independently by each bacterium as they adapted to their respective intracellular niches (Gottlieb et al. 2015). Intriguingly, C. burnetii encodes several intact or pseudogenized genes that encode the components of a Type IV pilus (Seshadri et al. 2013). A related Type IV pilus enables Acinetobacter baumannii to acquire DNA from the environment with high efficiency (Smith et al. 2007), indicating that during an earlier stage during $C$. burnetii's evolution, a functional Type IV pilus endowed it with the ability to acquire foreign genes proficiently, thereby likely facilitating its transition into a mammalian

pathogen from a tick-associated ancestor (Smith et al. 2015; Duron et al. 2015; Gerhart et al. 2016).

\section{HGT contributed to the lipopolysaccharide profile of $C$. burnetii}

HGT-origin genes are strewn all across the C. burnetii genome, indicating, as shown previously (Raghavan et al. 2008), that there are no prototypical pathogenicity islands, but, based on their clustered genome locations, several genes appear to have been acquired en bloc (Figure 2.1). A group of genes of particular interest is CBU_0678 to 
CBU_0683, which is part of an operon that encodes genes involved in the biosynthesis of LPS (Seshadri et al. 2003; Narasaki and Toman 2012). We conducted in-depth phylogenetic analyses of CBU_0678, the most upstream gene of this cluster in order to validate the HGTector results. Based on both Maximum Likelihood and Bayesian phylogenetic analyses (Figure 2.2, Figure 2.3), the closest orthologs of this gene are present in members of Alphaproteobacteria. Furthermore, O-polysaccharide biosynthesis genes tend to occur as an operon in most bacteria, and since several genes in this location (CBU_0673, CBU_0676, CBU_0678 to CBU_0682) were also likely acquired from Alphaproteobacteria (Figure 2.2), it is highly likely that the genes were acquired in a single event. Previous studies have shown that LPS genes are horizontally transferred among bacteria (Nelson and Selander 1994), probably due to their importance in hostpathogen interactions (Narasaki and Toman 2012). In fact, full-length LPS is the only $C$. burnetii virulence factor established in an immunocompetent animal model of infection. It protects the pathogen from innate immune response (Shannon et al. 2005), and avirulent Nine Mile phase II strain produces a severely truncated LPS due to the loss of 22 LPS biosynthesis genes, including CBU_0678 to CBU_0682 (Beare et al. 2006). The observation that important virulence factors in C. burnetii (e.g., LPS, TFSS, effector proteins) were assembled via HGT illustrates the significance of this process in the evolutionary history of this intracellular pathogen.

\section{HGT enhanced $C$. burnetii's fatty acid metabolism}

Another set of genes in C. burnetii that is of putative foreign origin is CBU_0034 to 


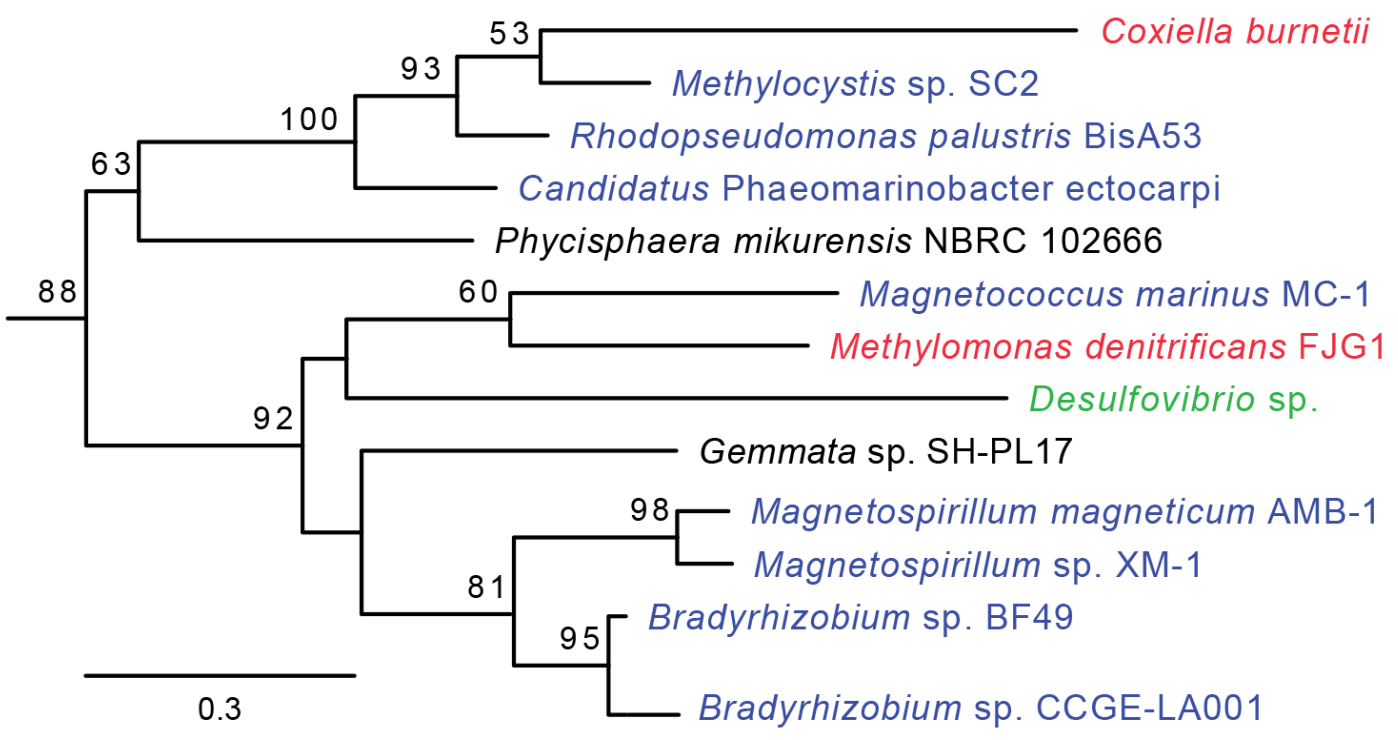

Figure 2.2 - Maximum Likelihood tree of CBU_0678, gained via HGT. A Maximum Likelihood phylogeny for CBU_0678, a LPS biosynthesis gene, is shown.

Gammaproteobacteria are in red, Alphaproteobacteria in blue, Delta/

Epsilonproteobacteria in green, and Planctomycetes in black. Bootstrap values of $>50$ are indicated at the nodes. Branch length measured in number of substitutions per site. The evolution model GTR $+\mathrm{I}+\mathrm{G}$ (General Time Reversible plus Invariant sites plus Gamma distribution) was selected using jModelTest2 (Darriba et al. 2012). Maximum Likelihood tree was constructed using RAxML (Stamatakis et al. 2008) as implemented in Geneious with 1,000 bootstrap replicates. 


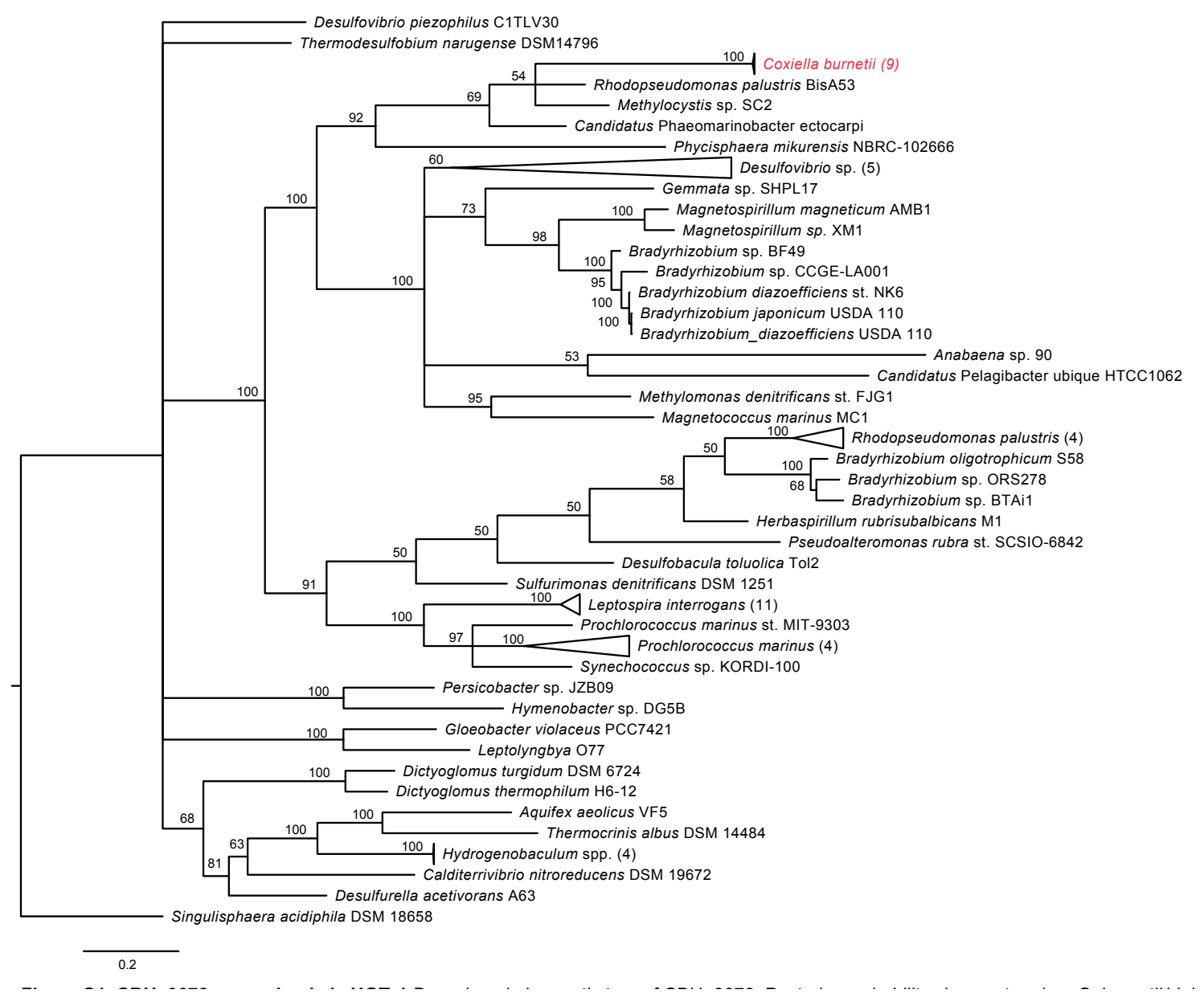

Figure 2.3 - Bayesian tree of CBU_0678, gained via HGT. A Bayesian phylogenetic tree of CBU_0678. Posterior probability shown at nodes. C. burnetii highlighted in red. Number of taxa collapsed into each branch is shown within parentheses. Branch length measured in number of substitutions per site. The evolution model GTR $+\mathrm{I}+\mathrm{G}$ (General Time Reversible plus Invariant sites plus Gamma distribution) was selected using jModelTest2 (Darriba et al. 2012). Bayesian tree was constructed using MrBayes as implemented in Geneious (Huelsenbeck and Ronquist 2001; Kearse et al. 2012). A chain length of 1,000,000 was used with a burn-in fraction of $25 \%$ and sampling every 100 trees. 
CBU_0038 (Figure 2.4). This gene cluster appears to have originated in either a Deltaproteobacteria or a Spirochete. We explored the HGT origin of this presumptive operon through phylogenetic analysis of its first gene, CBU_0038 (Figure 2.4A, Figure 2.5). Furthermore, this set of genes has a similar arrangement in both $C$. burnetii and in Spirochaeta africana DSM 8902, its best BLAST hit (Additional_file_A1.csv), indicating that the genes were transferred en bloc. In addition, an IS1111A transposase (CBU_0040) is proximally located to the operon, illustrating a probable role for this mobile genetic element in HGT (Figure 2.4B). Based on homology, CBU_0034 to CBU_0038 encode ACP, FabB, FabZ, FabA and FabH, respectively, which are involved in the synthesis of unsaturated fatty acids (Zhang and Rock 2008; Feng and Cronan 2009). In contrast, vertically inherited genes (CBU_0493 to CBU_0497) are responsible for the synthesis of saturated fatty acids (Gilk 2012), denoting that C. burnetii's ability to produce unsaturated fatty acids was enhanced through HGT. C. burnetii also contains a putative fatty acid desaturase (CBU_0920) that introduces double bonds into existing fatty acids (Gilk 2012). This gene also appears to be of HGT origin, but was not included in our analysis because it is present in other Legionellales. In addition to fatty acid biosynthesis genes, $C$. burnetii has also gained three pyruvate dehydrogenases (CBU_0686, CBU_0692, and CBU_0693) (Figure 2.1). Pyruvate dehydrogenase complex converts pyruvate into acetyl-CoA, which is a critical metabolite for both fatty acid biosynthesis and ATP generation (de Kok et al. 1998). Thus, HGT appears to have played a significant role in shaping $C$. burnetii's fatty acid metabolism. Furthermore, a recent study showed that transposon-mediated disruption of CBU_0035 and CBU_0038 
A

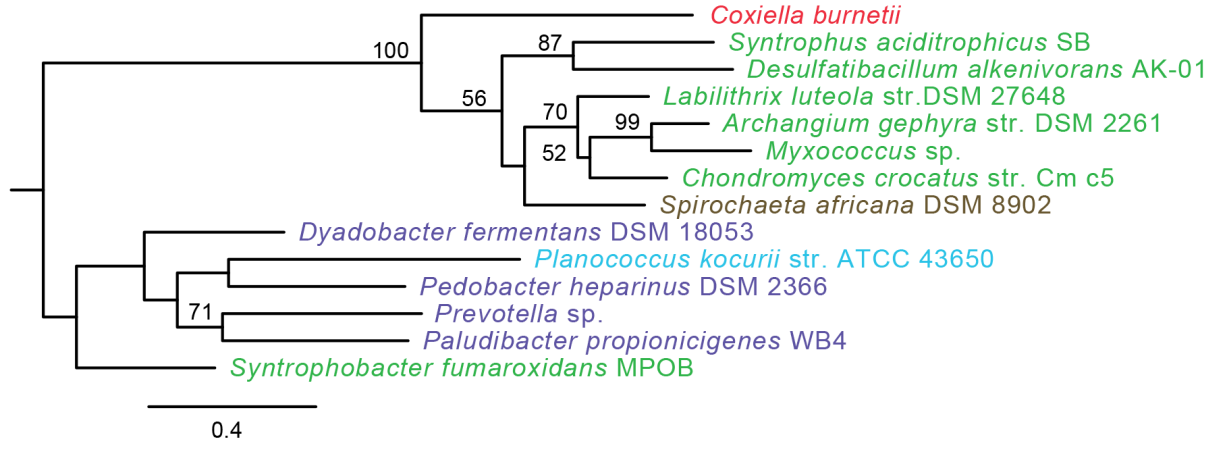

B

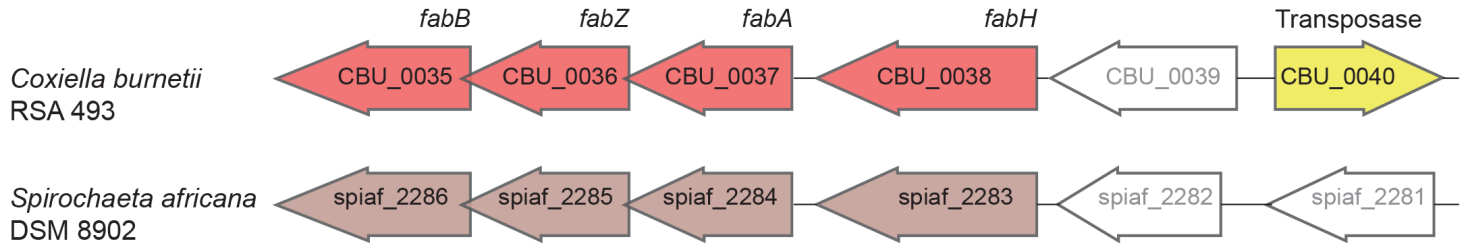

Figure 2.4 - A horizontally acquired fatty acid biosynthesis operon in C. burnetii.

(A) Maximum Likelihood phylogenetic tree for CBU_0038, a fatty acid biosynthesis gene. Gammaproteobacteria is colored red, Delta/Epsilonproteobacteria in green, Spirochaetes in brown, Bacteriodetes in purple, and Firmicutes in blue. Bootstrap values of $>50$ are indicated at the nodes. Branch length measured in number of substitutions per site. The evolution model GTR $+\mathrm{I}+\mathrm{G}$ (General Time Reversible plus Invariant sites plus Gamma distribution) was selected using jModelTest2 (Darriba et al. 2012). Maximum Likelihood tree was constructed using RAxML (Stamatakis et al. 2008) as implemented in Geneious with 1,000 bootstrap replicates. (B) Fatty acid biosynthesis genes have similar arrangement in C. burnetii and Spirochaeta africana, and an IS1111A transposase is located next to the operon in C. burnetii. 


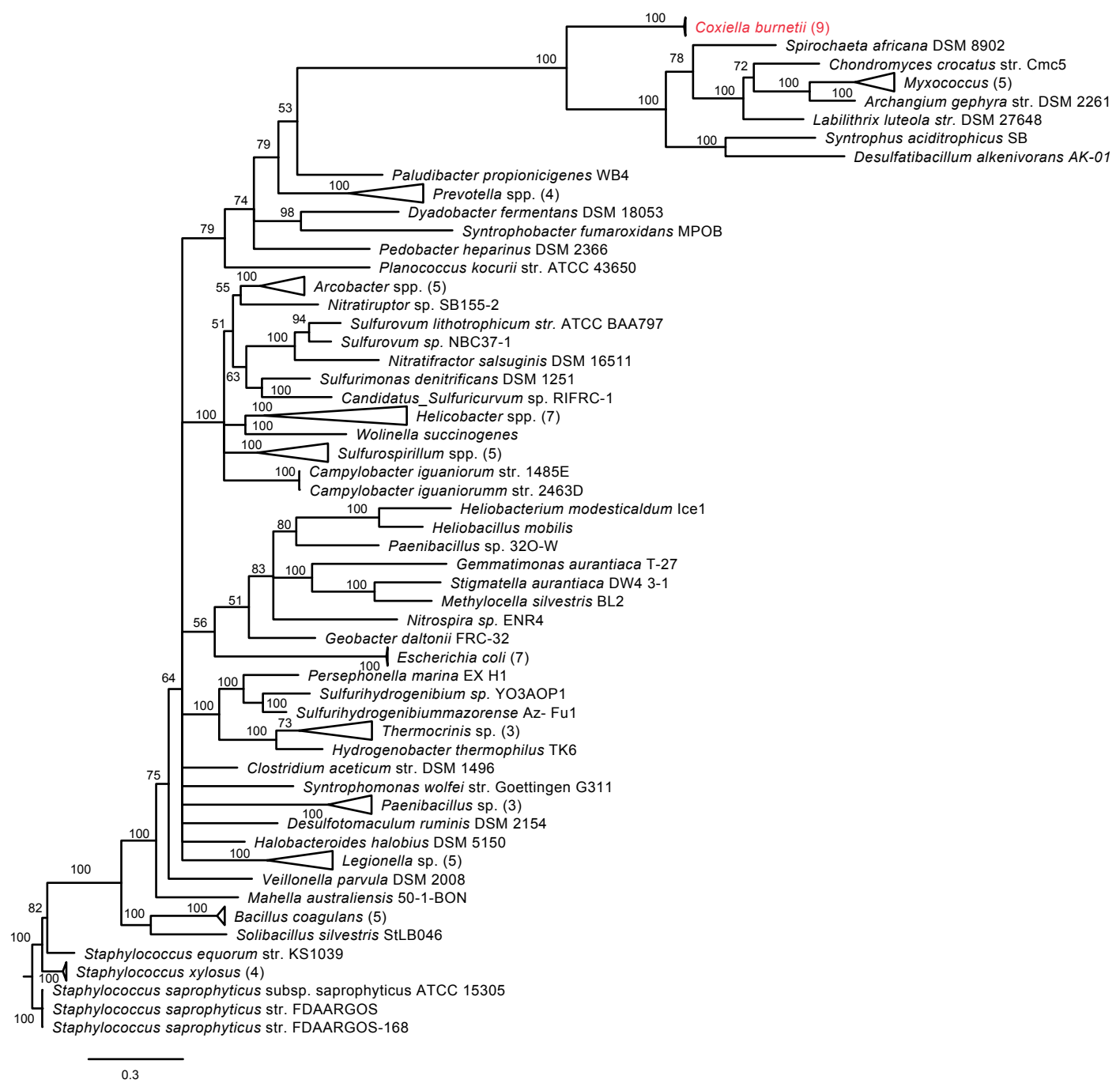

Figure 2.5 - Bayesian tree of CBU_0038, gained via HGT. A Bayesian phylogenetic tree of CBU_0038. Posterior probability shown at nodes. C. burnetii highlighted in red. Number of taxa collapsed into each branch is shown within parentheses. Branch length measured in number of changes per site. The evolution model GTR $+\mathrm{I}+\mathrm{G}(\mathrm{General}$ Time Reversible plus Invariant sites plus Gamma distribution) was selected using jModelTest2 (Darriba et al. 2012). Bayesian tree was constructed using MrBayes as implemented in Geneious (Huelsenbeck and Ronquist 2001; Kearse et al. 2012). A chain length of $1,000,000$ was used with a burn-in fraction of $25 \%$ and sampling every 100 trees. 
resulted in reduced $C$. burnetii growth within Vero cells, suggesting that this process is a critical constituent of the pathogen's physiology (Martinez et al. 2014).

\section{HGT enhanced $C$. burnetii's biotin metabolism}

Fatty acid biosynthesis enzymes require biotin as a cofactor, and biotin in turn is synthesized by utilizing a portion of the fatty acid biosynthesis pathway (Lin et al. 2010). Unlike most other bacteria, C. burnetii contains two copies of the gene bioC, the first committed step in biotin production. The two bioC genes are only $45 \%$ similar at the nucleotide level, indicating that they were not formed by a recent duplication event in $C$. burnetii. Further, bioC.2 (CBU_1004) has an 11 bp overlap with bioH and is part of the bioA-bioBFHCD-birA regulon (CBU_1008 to CBU_1002), whereas bioC.1 (CBU_0467) is a single gene located at a different part of the genome, indicating that bioC1 is of horizontal origin. To understand the functional relevance of these genes, we examined their expression levels in C. burnetii grown in ACCM-2 and within Vero cells (Warrier et al. 2014). The RNA-seq data revealed that all biotin biosynthesis genes (bioA, bioB, bioC.1, bioC.2, bioD, bioF, and bioH) were expressed under both conditions, but their expression was significantly higher in Vero cells than in ACCM-2 (Table 2.1). In contrast, the expression of $\operatorname{bir} A$, the transcriptional repressor of biotin operon showed the opposite pattern, suggesting that biotin is likely synthesized under both conditions, with possible upregulation within the host cell. Furthermore, a small molecule (MAC13772) that blocks biotin biosynthesis in $E$. coli (Zlitni et al. 2013) inhibited the growth of $C$. burnetii in ACCM-2, indicating that the production of biotin is a critical process in this 
Table 2.1 - Expression of biotin and heme biosyntheses genes in C. burnetii grown in ACCM-2 or Vero cells for $72 \mathrm{~h}$ measured using RNA-seq.

\begin{tabular}{lllll}
\hline Gene & $\begin{array}{l}\text { ACCM-2 } \\
\text { (LCV) }\end{array}$ & $\begin{array}{l}\text { Vero } \\
(\mathbf{L C V})\end{array}$ & $\begin{array}{l}\text { Fold } \\
\text { Change }\end{array}$ & $\begin{array}{l}\boldsymbol{p} \text {-value } \\
\text { (FDR corrected) }\end{array}$ \\
\hline Biotin & & & & \\
bioA & 2,093 & 3,915 & 1.87 & $<0.001$ \\
bioB & 1,562 & 10,118 & 6.48 & $<0.001$ \\
bioC. 1 & 9,659 & 15,678 & 1.62 & $<0.001$ \\
bioC. 2 & 1,562 & 2,909 & 1.86 & $<0.001$ \\
bioD & 1,590 & 6,233 & 3.92 & $<0.001$ \\
bioF & 1,159 & 5,354 & 4.62 & $<0.001$ \\
bioH & 916 & 4,836 & 5.28 & $<0.001$ \\
birA & 5,803 & 3,961 & 0.68 & 0.0055 \\
Heme & & & & \\
hem $A$ & 6,447 & 10,335 & 1.60 & 0.0007 \\
hemB & 49,632 & 5,778 & 0.12 & $<0.001$ \\
hem $C$ & 6,652 & 4,783 & 0.72 & 0.0183 \\
hemD & 4,534 & 6,420 & 1.42 & 0.0110 \\
hemE & 29,442 & 16,762 & 0.57 & $<0.001$ \\
hem $F$ & 9,104 & 12,200 & 1.34 & 0.0291 \\
hem $H$ & 3,343 & 8,085 & 2.42 & $<0.001$ \\
hem $K$ & 3,497 & 6,141 & 1.76 & $<0.001$ \\
\hline & & & &
\end{tabular}


pathogen (Figure 2.6). Biotin synthesis has been shown to be critical to the virulence of other human pathogens such as Francisella tularensis and Mycobacterium tuberculosis (Woong Park et al. 2011; Feng et al. 2014). Thus, novel pharmaceutical agents that block this process hold promise as a potential broad-spectrum agent to treat intracellular pathogens.

\section{Heme biosynthesis is an essential metabolic process in $C$. burnetii}

C. burnetii's genome is small compared to that of free-living bacteria such as E. coli ( $\sim 2$ $\mathrm{Mb}$ and $\sim 5 \mathrm{Mb}$, respectively). Correspondingly, it contains less than half the number of tRNAs than in E. coli (42 and 89, respectively). This reduction has occurred due to loss of redundant tRNAs; for instance, while E. coli has five copies of tRNA ${ }^{\text {Ile }}, C$. burnetii has only one copy. In contrast to other tRNAs, $C$. burnetii contains an additional tRNA ${ }^{\text {Glu }}$ isoacceptor ( $\left(\mathrm{RNA}^{\mathrm{Glu}} 2\right.$, anticodon CUC) that is not found in any other Gammaproteobacteria, denoting that it was gained via HGT (Figure 2.7A). Additionally, a toxin-antitoxin system (CBU_0284, CBU0285) that was probably horizontally acquired is located adjacent to tRNA ${ }^{\mathrm{Glu}} 2$, further strengthening the evidence for its putative HGT origin (Figure 2.7B). Because tRNA ${ }^{\text {Glu }} 2$ is retained in such a streamlined genome, it most likely has a critical function; in fact, a tRNA ${ }^{\text {Glu}} 2$-deletion strain had significantly slower growth than the wild-type strain in ACCM-2, which was restored in a complementation strain, signifying the importance of the HGT-derived tRNA to optimum bacterial fitness (Figure 2.6). However, the major function of tRNA ${ }^{\mathrm{Glu}} 2$ is unlikely to be protein synthesis because tRNA ${ }^{\text {Glu}} 2$ (anticodon CUC) cannot decode the major glutamate 


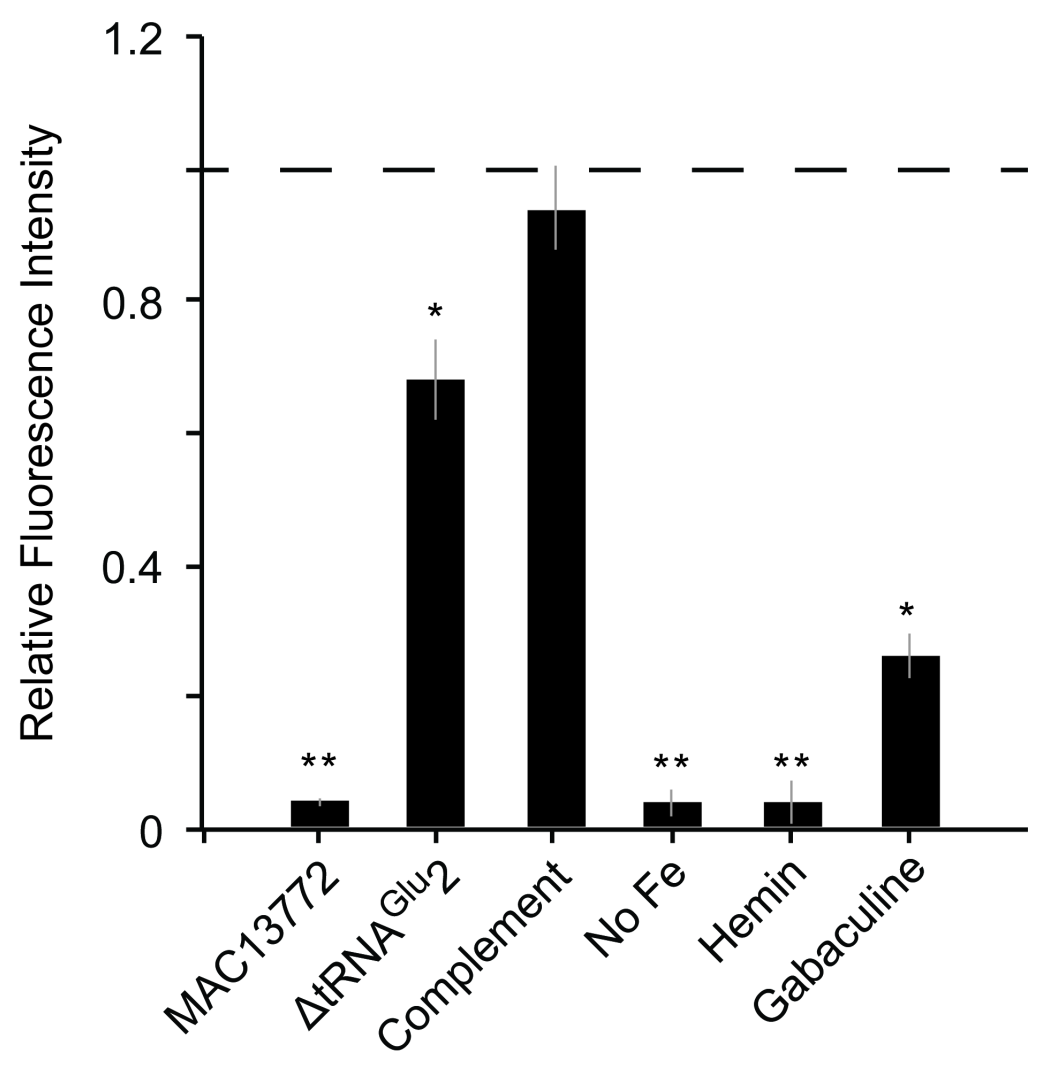

Figure 2.6 - Heme and biotin syntheses effect on $C$. burnetii's growth. Growth of $C$. burnetii in ACCM-2 after 7 days was measured using PicoGreen. Fluorescence of each strain relative to that of control (wild-type grown in ACCM-2; dashed line) is shown. MAC13772: ACCM-2 supplemented with $300 \mu \mathrm{g} / \mathrm{ml}$ MAC13772, a biotin biosynthesis inhibitor; tRNAGlu2: tRNAGlu2-deletion strain; Complement: tRNAGlu2-deletion complemented with intact tRNAGlu2 on pAM100; No Fe: ACCM-2 without FeSO4; Hemin: ACCM-2 with hemin in place of FeSO4; Gabaculine: ACCM-2 supplemented with $100 \mu \mathrm{M}$ gabaculine, a heme biosynthesis inhibitor. Statistically significant differences in growth from control are indicated by $\left({ }^{* *}\right) \mathrm{p}<0.001$ and $\left({ }^{*}\right) \mathrm{p}<0.01$ (unpaired t-test). 
A

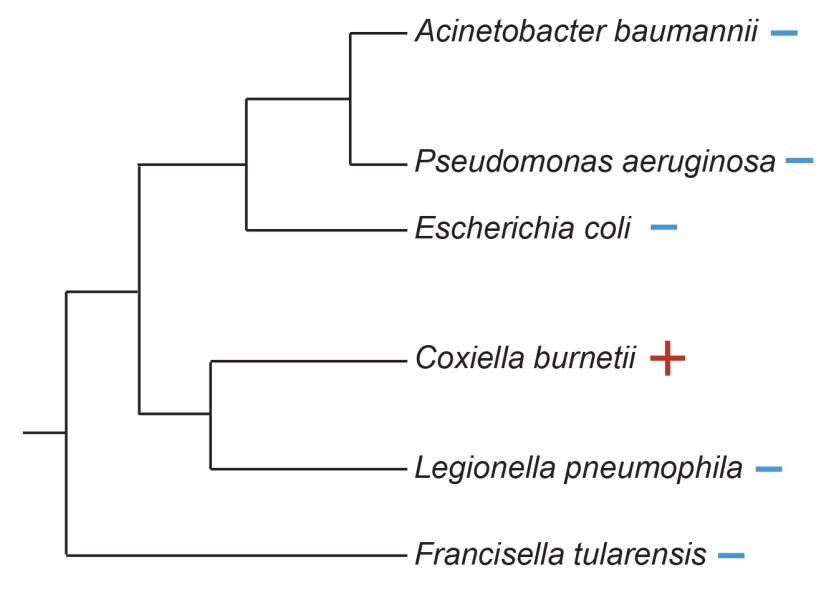

B

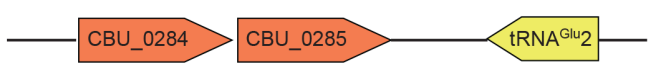

Toxin/antitoxin system

Figure 2.7 - tRNA ${ }^{\text {Glu}} 2$ was horizontally acquired by $\boldsymbol{C}$. burnetii. (A) $16 \mathrm{~S}$ rDNA

Neighbor-Joining phylogenetic tree of Gammaproteobacteria showing the presence $(+)$ or absence (-) of tRNA ${ }^{\mathrm{Glu}}$ (anticodon CUC). (B) A putative toxin/antitoxin system is located adjacent to tRNA ${ }^{\mathrm{Glu}} 2$. 
codon GAA (76\%), whereas tRNA ${ }^{\text {Glu }} 1$ (anticodon UUC) can decode both GAA and GAG codons efficiently. Additionally, Coxiella proteins are not enriched for glutamate in comparison to E. coli ( $\sim 6 \%$ of all codons is for Glu in both bacteria). Taken together, these data suggest that protein biosynthesis is not tRNA ${ }^{\text {Glu }} 2$ 's major function in $C$. burnetii.

An alternate function for tRNA ${ }^{\mathrm{Glu}} 2$ is in heme biosynthesis. Heme is an ironcontaining tetrapyrrole that serves multiple cellular functions, including in respiration, energy generation, oxidative reactions and signal transduction (Almiron et al. 2001). The universal precursor of tetrapyrrole biosynthesis is 5-aminolevulinic acid (ALA) (Anzaldi and Skaar 2010). There are two alternate pathways in nature for the synthesis of ALA: C5 pathway found in most bacteria, and Shemin pathway present in most eukaryotes, including humans (Frankenberg et al. 2003). The starting point of the C5 pathway is Glutamyl-tRNA ${ }^{\text {Glu }}$, which is converted into ALA using two consecutive enzymes HemA and HemL. In Shemin pathway, ALA is synthesized from Succinyl-CoA and glycine by the enzyme ALAS (Frankenberg et al. 2003). The remaining steps are shared between the C5 and Shemin pathways. We examined the C. burnetii genome and discovered that it encodes an intact C5 heme biosynthesis pathway, and RNA-seq data confirmed that these genes are expressed during growth (Table 2.1). Additionally, heme biosynthesis pathway is conserved in all C. burnetii strains but is absent in non-pathogenic Coxiella present in ticks, and a $C$. burnetii strain with a transposon insertion in the hemL gene had reduced growth in Vero cells (Martinez et al. 2014), implying the importance of heme biosynthesis to the human pathogen. 
Several bacteria augment heme biosynthesis with heme transported from the outside (Mike et al. 2013). C. burnetii encodes a transporter ( $f e o A B)$ for ferrous iron, which is required for heme biosynthesis, but has no known transporters for ferric ions or heme. Consequently, we reasoned that the pathogen might not be able to utilize extracellular heme as its sole source of iron. To test this, we inoculated equal amounts of Coxiella into ACCM-2 medium with FeSO4 (standard recipe) or into ACCM-2 in which FeSO4 was replaced with equimolar amount of hemin. Growth was measured after 7 days using PicoGreen as described previously (Martinez et al. 2015). As shown in Figure 2.6, Coxiella growth in FeSO4-containing ACCM-2 was significantly higher than in hemincontaining ACCM-2 ( $p<0.001$, paired t-test), whereas the fluorescence measurements between the control (ACCM-2 without FeSO4) and the hemin-containing ACCM-2 samples were not significantly different $(\mathrm{p}>0.05$, paired t-test), showing that $C$. burnetii cannot utilize external heme. To test $C$. burnetii's requirement for heme biosynthesis, we treated C. burnetii with increasing concentrations of gabaculine, an inhibitor of HemL (Wang et al. 1997), and found that $100 \mathrm{uM}$ gabaculine significantly inhibited bacterial growth ( $\mathrm{p}<0.001$, paired t-test) (Figure 2.6). These data show the probable of heme biosynthesis to C. burnetii's normal physiology, and indicates that heme biosynthesis genes are potential targets for the development of new anti-Coxiella therapies. 


\section{CHAPTER 3}

Coxiella burnetii and Leishmania mexicana residing within similar parasitophorous vacuoles elicit disparate host responses

\subsection{ABSTRACT}

Coxiella burnetii is a bacterium that thrives in an acidic parasitophorous vacuole (PV) derived from lysosomes. Leishmania mexicana, a eukaryote, has also independently evolved to live in a morphologically similar PV. As Coxiella and Leishmania are highly divergent organisms that cause different diseases, we reasoned that their respective infections would likely elicit distinct host responses despite producing phenotypically similar parasite-containing vacuoles. The objective of this study was to investigate, at the molecular level, the macrophage response to each pathogen. Infection of THP-1 (human monocyte/macrophage) cells with Coxiella and Leishmania elicited disparate host responses. At 5 days post-infection, 1057 genes were differentially expressed (746 genes up- and 311 genes down-regulated) in C. burnetii infected cells when compared to uninfected cells, whereas 698 genes (534 genes up- and 164 genes down-regulated) were differentially expressed in L. mexicana infected cells. Interestingly, of the 1755 differentially expressed genes identified in this study, only 126 genes $(\sim 7 \%)$ are common to both infections. We also discovered that 1090 genes produced mRNA isoforms at significantly different levels under the two infection conditions, suggesting that alternate proteins encoded by the same gene might have important roles in host response to each 
infection. Additionally, we detected 257 micro RNAs (miRNAs) that were expressed in THP-1 cells and identified miRNAs that were specifically expressed during Coxiella or Leishmania infections. Collectively, this study identified host mRNAs and miRNAs that were influenced by Coxiella and/or Leishmania infections, and our data indicate that although their PVs are morphologically similar, Coxiella and Leishmania have evolved different strategies that perturb distinct host processes to create and thrive within their respective intracellular niches. 


\subsection{INTRODUCTION}

Macrophages that phagocytize pathogens and recruit other immune cells are critical for the elimination of potential infections. Within macrophages, engulfed pathogens are transported inside phagosomes that later fuse with lysosomes to generate the phagolysosome. Most pathogens are degraded within the phagolysosome, which has a very harsh environment (low $\mathrm{pH}$, high concentration of lysosomal hydrolases, presence of cationic peptides etc.) (Kinchen and Ravichandran 2008; Flannagan et al. 2009). Several pathogens have evolved strategies to survive and replicate within macrophages: Toxoplasma gondii prevents the fusion of its vacuoles with the endosomal pathway; Salmonella enterica Typhimurium, Mycobacterium tuberculosis and Legionella pneumophila block maturation of phagosomes into phagolysosomes; Shigella flexneri and Listeria monocytogenes escape into cytoplasm from phagosomes before lysosomal fusion; Trypanosoma cruzi escapes from phagosomes after fusion with lysosomes (Flannagan et al., 2009; Swanson and Fernandez-Moreia, 2002).

Unlike most other pathogens, Coxiella (a bacterium) and Leishmania (a eukaryote) have independently evolved the ability to thrive in a parasitophorous vacuole (PV) that is derived from the fusion of phagosomes with lysosomes (Voth and Heinzen 2007; Alix et al. 2011). Coxiella burnetii (the only defined species within this genus) causes human Q fever and chronic endocarditis. The bacterium is shed in milk, urine, and birth products of animals, and can survive in the environment via a "spore-like" form called the small cell variant (SCV). C. burnetii is usually acquired via inhalation, and 
initially infects alveolar macrophages but then spreads to mononuclear phagocytes of other tissues. Within the macrophage, SCV transforms into a metabolically active form called the large cell variant (LCV), and multiple Coxiella-containing vacuoles merge to form a single large vacuole that fuses with endolysosomal vesicles to give rise to the mature Coxiella PV (van Schaik et al. 2013).

Leishmania is a genus of trypanosomatid parasite that comprises several species of medical and veterinary importance that cause cutaneous, mucocutenous, or visceral diseases. It has a dimorphic lifecycle that alternates between an extracellular promastigote form in insect vectors and an intracellular amastigote form in mammalian hosts (Herwaldt 1999). The primary host cells of Leishmania are macrophages, but it can also infect neutrophils, fibroblasts and dendritic cells (Contreras et al. 2014). Similar to the biogenesis of Coxiella PV, the Leishmania-containing vacuole also fuses with endolysosomal vesicles to give rise to the mature Leishmania PV. However, the morphology of PV varies among different Leishmania species. In several species, including $L$. donovani, $L$. infantum, and L. major, only one or two amastigotes reside within each PV, which segregates into new vacuoles after parasite replication. In contrast, as observed for Coxiella PVs, parasites of the Leishmania mexicana complex such as $L$. mexicana and L. amazonensis form communal PVs that continuously enlarge as the parasites replicate (Real et al. 2010). Interestingly, coinfection studies have shown that PVs formed by L. amazonensis amastigotes can fuse with C. burnetii PVs but not with PVs containing L. major amastigotes, suggesting that the intracellular niches generated by Leishmania mexicana complex parasites and Coxiella may be compositionally rather 
similar (Real et al. 2010; Beare et al. 2011; Veras et al. 1995; Rabinovitch and Veras 1996; Newton and Roy 2011).

Both Coxiella and Leishmania actively participate in the creation of their respective PVs (Voth and Heinzen 2007; Waller and McConville 2002). To begin to understand how the two distantly related pathogens generate phenotypically similar PVs, we compared host gene expression in human macrophage cells (THP-1) infected with either C. burnetii or L. mexicana. Our data show that the bacterium and the eukaryote elicit distinct host messenger RNA (mRNA) and microRNA (miRNA) responses, indicating that despite their superficial similarity, generation and maintenance of the Coxiella PV and Leishmania PV involve distinct host processes. 


\subsection{MATERIALS AND METHODS}

\section{C. burnetii and $L$. mexicana infection of THP-1 cells, RNA extraction and RNA-seq}

THP-1 human macrophage cells (TIB-202; ATCC) were maintained in RPMI 1640 medium (Gibco) supplemented with $10 \%$ fetal calf serum (Gibco) at $37^{\circ} \mathrm{C}$ in $5 \% \mathrm{CO}_{2}$. Cells were incubated in the presence of $200 \mathrm{nM}$ phorbol 12-myristate 13-acetate (PMA; EMD Biosciences) for $24 \mathrm{~h}$ to induce differentiation into adherent, macrophage-like cells. Prior to infection, PMA-containing medium was replaced with fresh RPMI without PMA. Cells were infected with either C. burnetii (Nine Mile phase II, RSA 493) or promastigotes of L. mexicana (MNYZ/BZ/62/M379) at an approximate multiplicity of infection of 25 and incubated for five days. Growth medium was replaced every two days and formation of Coxiella and Leishmania PVs was monitored microscopically. At $5 \mathrm{~d}$ post-infection, growth medium was replaced with $1 \mathrm{ml}$ of TRI reagent (Life Technologies) and total RNA was extracted, genomic DNA was removed by DNAse (Life Technologies) treatment, as per instructions. RNA from two samples each of uninfected, Coxiella-infected, and Leishmania-infected THP-1 cells were used to prepare mRNA and small RNA Illumina sequencing libraries. To analyze gene expression, the six mRNA libraries were pooled into a single lane of an Illumina HiSeq 2000 (2x75 cycles). For miRNA identification, the six small RNA libraries were pooled into a single Illumina Miseq lane (1x50 cycles). All RNA-seq reads are available at National Center for Biotechnology Information Sequence Read Archive (Accession SRP045986). 


\section{Mapping sequencing reads and identification of differentially expressed genes}

Adapters were removed from reads and were filtered by quality $(>\mathrm{Q} 20)$ and length (>50bp) using Trimmomatic v0.30 (Bolger et al. 2014). Homo sapiens reads were filtered for possible contamination by mapping to C. burnetii genome (NC_002971.3) using BWA MEM v0.7.5 (Li and Durbin 2010) and L. mexicana genome (NZ_CADB00000000.1) using Tophat v2.0.11 (Kim et al. 2013) and were removed. After processing, reads were mapped to Homo sapiens Genome Reference Consortium Human Build 37 (GCF_000001405.13) using CLC Genomic Workbench v6.5. To identify differential gene expression, replicate data were pooled for pairwise comparisons and quantile normalized using CLC Genomic Workbench v6.5. Genes were filtered based on at least 10 raw reads mapping to each sample, and a $\log 2$ transformed fold change of one standard deviation above or below the mean. Differentially expressed genes were chosen based on significance ( $\mathrm{P}<0.05$, FDR-corrected beta-binomial distribution test). Raw read counts mapped to each mRNA isoform were exported from CLC into EBSeq (Leng et al. 2013) and differential expression of isoforms was determined based on significant EBSeq values ( $\mathrm{P}<0.05$, FDR-corrected).

For quantitative PCR (qPCR) validation of gene expression, $1 \mu \mathrm{g}$ of DNasetreated RNA and oligo-dT primers were used to prepare cDNA (Thermo Scientific). A subset of genes involved in host cell death (TGFB2, RIPK2, CYR61, CYP1B1, NFKBIA) was selected and qPCR was performed using SYBR green on an Agilent

Mx3000P System. Fold difference value for each gene was calculated using the $2^{-\Delta \Delta C T}$ method with GAPDH as the control (Livak and Schmittgen 2001). To assess the 
correlation between expression estimates from RNA-seq and qPCR [As shown previously (Raghavan et al. 2012)], we calculated the Pearson correlation coefficient between fold difference values calculated by each method for Coxiella- and Leishmania-infected cells.

\section{Gene Ontology (GO) analysis and protein-protein interaction networks}

Gene Ontology (GO) terms were found using Database for Annotation, Visualization and Integrated Discovery (DAVID) and the GO FAT filter. GO-term enrichment tests were also performed with DAVID (Huang et al. 2009a, 2009b). Kyoto Encyclopedia of Genes and Genomes (KEGG) pathways over-represented among differentially expressed genes were chosen based on the level of statistical significance $(\mathrm{P}<0.01)$. Protein-protein interaction networks were visualized using STRING 9.1 (Franceschini et al. 2013). Proteins unconnected to the main graph were removed. Markov Clustering was performed on STRING confidence scores using an inflation factor of 2 to visualize subgraphs of interacting protein processes (Brohée and van Helden 2006). GO-terms were overlaid onto the graphs using STRING to identify what processes were represented in the separate subgraphs.

\section{Identification of miRNAs}

Sequencing reads were cleaned by removing adapters and filtered by quality $(>\mathrm{Q} 20)$ and length (>15bp) using Trimmomatic (Bolger et al. 2014). Replicate data was pooled and miRNAs were identified using CLC based on having an average of at least 10 reads 
mapped to mature 5' or 3' miRNAs annotated in mirBase (Kozomara and Griffiths-Jones 2014). 


\subsection{RESULTS AND DISCUSSION}

\section{C. burnetii and $L$. mexicana infections induce robust but non-overlapping host responses}

Human monocyte/macrophage cell line THP-1 was used to evaluate host responses against $C$. burnetii and L. mexicana. Previous studies have investigated host responses during early stages ( $6 \mathrm{~h}$ pi to $72 \mathrm{~h}$ pi) of infections by $C$. burnetii and by various Leishmania species (Ren et al. 2003; Mahapatra et al. 2010; Rabhi et al. 2013, 2012; de Muylder et al. 2011); however, because the transformation from the infective form (SCV and promastigote, respectively) to the replicative form (LCV and amastigote, respectively) occur at differing rates in the two pathogens, we analyzed a later point during infection (5d pi) when both pathogens have generated large PVs that fill most of the host cell volume. When compared to uninfected THP-1 cells, 1057 genes (746 upand 311 down-regulated) were differentially expressed in C. burnetii infected THP-1 cells, whereas 698 genes (534 up- and 164 down-regulated) were differentially expressed in L. mexicana infected cells (Figure 3.1, Additional_file_A2.csv, Additional_file_A3.csv). Interestingly, the sets of genes affected by the two pathogens are very different. Of the 1755 total genes identified in this study, only 126 genes $(\sim 7 \%)$ are differentially expressed under both conditions, and no metabolic pathways were significantly enriched within this common set of genes (Figure 3.1,

Additional_file_A4.csv). A previous study that compared THP-1 cell response to infections by Coxiella and Chlamydia trachomatis (an intracellular bacterium), reported 
A

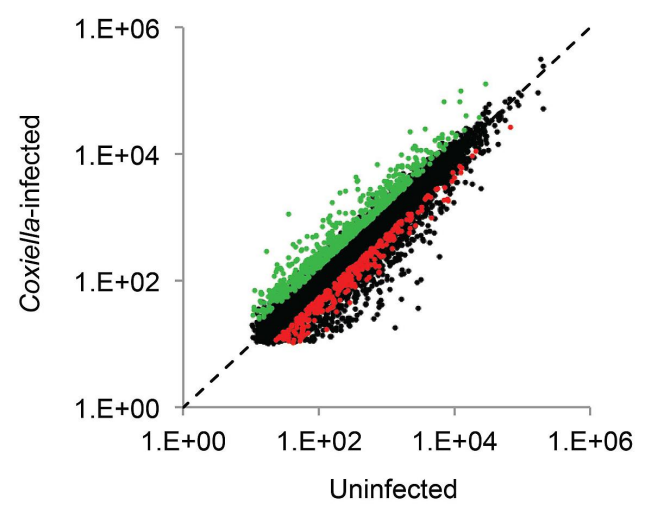

B

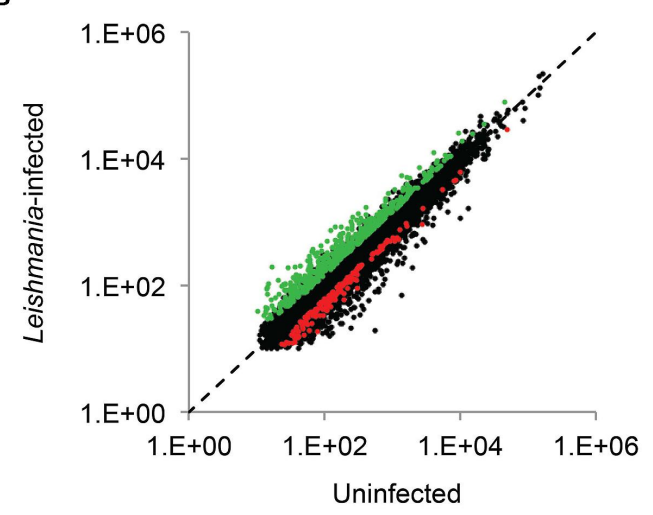

c

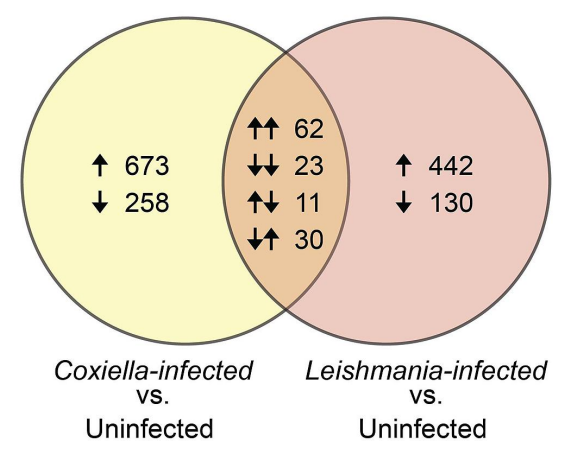

Figure 3.1 - Identification of differentially expressed genes. Gene expression in (A) Coxiella burnetii-infected and (B) Leishmania mexicana-infected THP-1 cells in comparison to uninfected THP-1 cells are shown. Differentially expressed genes are highlighted in red and green. (C) Comparison of genes differentially expressed in $C$. burnetii-infected and L. mexicana-infected cells. Arrows indicate up-regulation or downregulation of genes. 
an overlap of $\sim 25 \%$ of genes differentially expressed between the two infections (Ren et al. 2003). The low overlap between the host responses to Coxiella and Leishmania, and the higher magnitude of differentially expressed genes of host response to C. burnetii than that to L. mexicana possibly reflects the more distant evolutionary relationship between the bacteria and the eukaryotic parasite compared to the two bacterial pathogens previously studied. Apoptosis and host cell immune response pathways were the most significantly enriched KEGG pathways in Coxiella infected cells (Table 3.1), as observed in previous microarray-based studies (Ren et al. 2003; Mahapatra et al. 2010). Repression of host cell death by Coxiella has been reported previously (Voth et al. 2007; Lührmann and Roy 2007), and is thought to promote intracellular growth of Coxiella within large PVs; conversely, induction of Toll-like Receptor signaling pathways and production of cytokines and chemokines participate in the host response to Coxiella infection (Zamboni et al. 2004; Graham et al. 2013).

In Leishmania-infected cells, purine metabolism was the only KEGG pathway that was significantly perturbed (Table 3.1). Leishmania is dependent on host for its purine supply (McConville et al. 2007), and three genes (ADSSL1, RRM2, PRPS1) involved in purine biosynthesis or salvage pathways were significantly overexpressed in infected THP-1 cells. Intriguingly, a majority of "purine metabolism" genes listed in Table 3.1 regulate the levels of intracellular second messengers cAMP and cGMP. Adenylate cyclases (ADCY4, ADCY8, ADCY9, ADCY10) catalyze the formation of cAMP from ATP; guanylate cyclase (GUCY1A2) catalyzes the conversion of GTP to cGMP; phosphodiesterases (PDE4C, PDE6G, PDE2A) catalyze the hydrolysis of cAMP 
Table 3.1 - KEGG pathways enriched in Coxiella-infected and Leishmania-infected THP-1 cells

\begin{tabular}{|c|c|c|c|c|c|}
\hline Sample & $\begin{array}{l}\text { KEGG } \\
\text { Term }\end{array}$ & Description & Genes & $\begin{array}{l}\text { Fold } \\
\text { Change }\end{array}$ & P Value \\
\hline \multirow[t]{7}{*}{$\begin{array}{l}\text { Coxiella- } \\
\text { infected }\end{array}$} & hsa04210 & Apoptosis & $\begin{array}{l}\text { BID, IRAK2, TNF, XIAP, RELA, } \\
\text { TP53, NFKBIA, ENDOD1, NFKB1, } \\
\text { BIRC3, TNFRSF10A, CASP10, } \\
\text { PRKAR2B, IRAK3, TNFRSF10B, } \\
\text { PPP3CC, IL1B, PIK3R5, PIK3R3, } \\
\text { IL1A }\end{array}$ & 3.51 & $2.1 \mathrm{E}-06$ \\
\hline & hsa04621 & $\begin{array}{l}\text { NOD-like } \\
\text { receptor } \\
\text { signaling } \\
\text { pathway }\end{array}$ & $\begin{array}{l}\text { CXCL1, TNF, XIAP, IL8, RELA, } \\
\text { CXCL2, NFKBIA, NFKB1, BIRC3, } \\
\text { NOD2, RIPK2, IL1B, TNFAIP3 }\end{array}$ & 3.20 & $5.4 \mathrm{E}-04$ \\
\hline & hsa04060 & $\begin{array}{l}\text { Cytokine- } \\
\text { cytokine } \\
\text { receptor } \\
\text { interaction }\end{array}$ & $\begin{array}{l}\text { CXCL1, TNFRSF21, CCL3, TNF, } \\
\text { CXCL5, CXCL3, CXCL2, TNFSF15, } \\
\text { CXCL6, IL7R, CCL4, TGFB2, LIF, } \\
\text { CCL22, IL23A, CCL20, CCL3L1, } \\
\text { IL4R, TNFRSF18, IL15RA, IL1B, } \\
\text { IL1A, BMP2, IL8, CD40, IL11RA, } \\
\text { TNFRSF10A, INHBA, ACVR2B, } \\
\text { TNFRSF10B, VEGFA }\end{array}$ & 1.81 & $1.6 \mathrm{E}-03$ \\
\hline & hsa04062 & $\begin{array}{l}\text { Chemokine } \\
\text { signaling } \\
\text { pathway }\end{array}$ & $\begin{array}{l}\text { CXCL1, ADCY4, CCL3, LYN, } \\
\text { CXCL5, IL8, HCK, CXCL3, RELA, } \\
\text { CXCL2, NFKBIA, ADRBK2, } \\
\text { NFKB1, CXCL6, CCL4, CCL22, } \\
\text { CCL20, CCL3L1, GNG10, SOS2, } \\
\text { PIK3R5, GNB4, PIK3R3, GNG7 }\end{array}$ & 1.96 & $2.2 \mathrm{E}-03$ \\
\hline & hsa05222 & $\begin{array}{l}\text { Small cell } \\
\text { lung cancer }\end{array}$ & $\begin{array}{l}\text { E2F1, TRAF1, XIAP, PTGS2, RELA, } \\
\text { TP53, ITGA2, NFKBIA, NFKB1, } \\
\text { BIRC3, LAMB3, PIK3R5, PIK3R3, } \\
\text { TRAF3 }\end{array}$ & 2.55 & $2.8 \mathrm{E}-03$ \\
\hline & hsa05200 & $\begin{array}{l}\text { Pathways in } \\
\text { cancer }\end{array}$ & $\begin{array}{l}\text { TRAF1, E2F1, BID, PTGS2, XIAP, } \\
\text { STAT5A, MITF, NFKBIA, NFKB1, } \\
\text { NFKB2, TCF7L2, MMP1, TGFB2, } \\
\text { LAMB3, SOS2, PIK3R5, CCNA1, } \\
\text { PIK3R3, FGF2, TRAF3, BMP2, IL8, } \\
\text { VHL, RELA, TP53, ITGA2, BIRC5, } \\
\text { BIRC3, FZD4, DAPK3, CTNNA3, } \\
\text { RAD51, SMO, ETS1, VEGFA }\end{array}$ & 1.63 & $4.2 \mathrm{E}-03$ \\
\hline & hsa04620 & $\begin{array}{l}\text { Toll-like } \\
\text { receptor } \\
\text { signaling } \\
\text { pathway }\end{array}$ & $\begin{array}{l}\text { CCL3, TNF, IL8, RELA, NFKBIA, } \\
\text { NFKB1, CD40, CCL4, CD86, } \\
\text { MAP3K8, IL1B, PIK3R5, PIK3R3, } \\
\text { CD14, TRAF3 }\end{array}$ & 2.27 & $5.5 \mathrm{E}-03$ \\
\hline $\begin{array}{l}\text { Leishmania } \\
\text {-infected }\end{array}$ & hsa00230 & $\begin{array}{l}\text { Purine } \\
\text { metabolism }\end{array}$ & $\begin{array}{l}\text { ADCY4, ADSSL1, ADCY8, POLA1, } \\
\text { PDE4C, PDE6G, POLE4, PDE2A, } \\
\text { ADCY9, RRM2, PKLR, GUCY1A2, } \\
\text { ADCY10, PRPS1 }\end{array}$ & 2.40 & $5.1 \mathrm{E}-03$ \\
\hline
\end{tabular}


and/or cGMP. Previous studies have shown that Leishmania resists host antimicrobial activities by modulating several host signaling pathways, including $\mathrm{Ca}^{2+}$ - and $\mathrm{PKC}$ dependent pathways, JAK-STAT pathways, and MAP kinases (Olivier et al. 2005). Similarly, Leishmania could be subverting the host's cAMP and cGMP signaling pathways in order to suppress immune responses and to promote its intracellular growth.

A protein-protein interaction network analysis using the STRING database (Franceschini et al. 2013) confirmed that Coxiella infection induced the expression of genes involved in negative regulation of cell death (Figure 3.2A). In contrast, this analysis identified that genes involved in positive regulation of cell death were upregulated in Leishmania-infected cells (Figure 3.2B). We confirmed this trend by analyzing the expression of a subset of cell death-related genes using qPCR (Figure 3.3). The induction of host cell death during later stages of infection probably aids in the cellto-cell transfer of Leishmania amastigotes within membrane blebs, as shown recently (Real et al. 2014).

\section{Differential expression of mRNA isoforms in infected and uninfected cells}

In human cells, alternate splicing of pre-mRNA can give rise to several isoforms of the mature mRNA, and proteins derived from them may have distinct cellular roles (Lareau et al. 2004). In addition to expanding the proteome, cells utilize alternate splicing as a regulatory tool. For example, a short splice variant of human tryptophan-tRNA synthase, but not the full length protein, regulates angiogenesis (Wakasugi et al. 2002). Isoform generation may also have a role in host cell response against infections. Different 
A

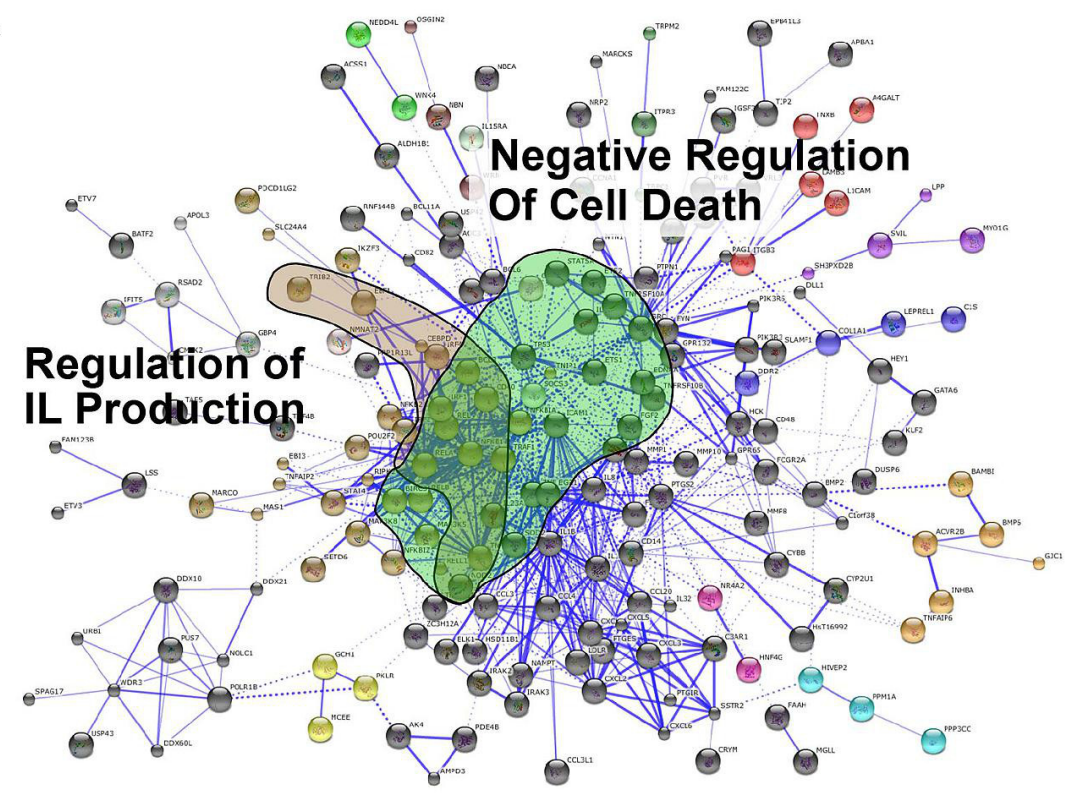

B

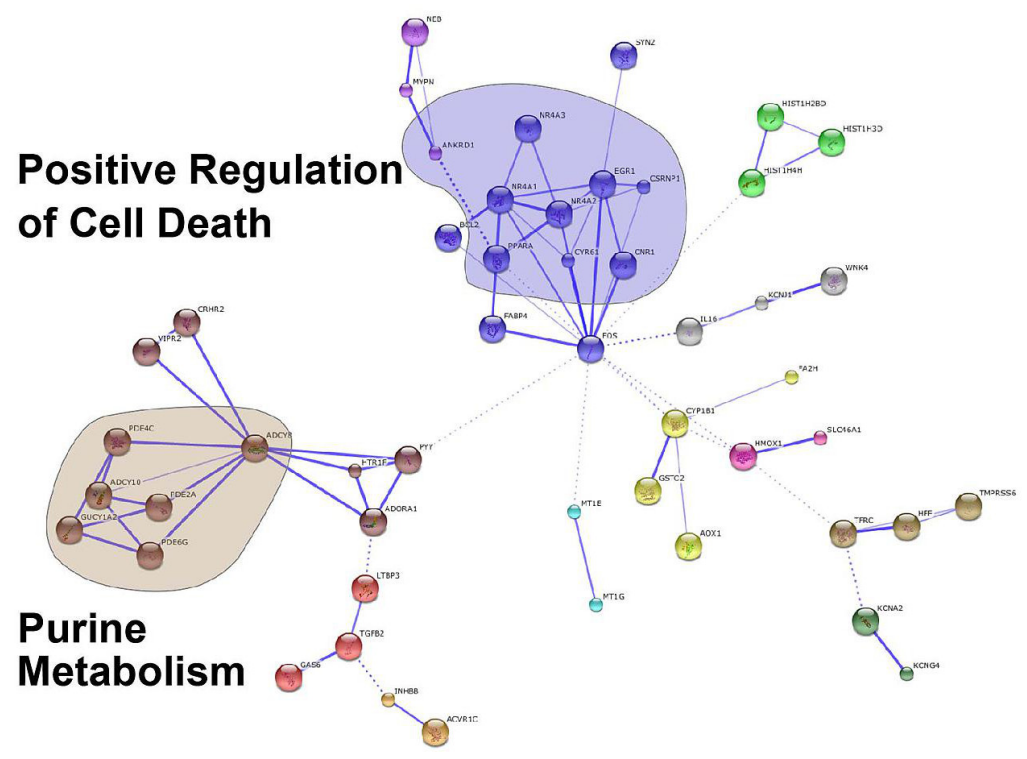

Figure 3.2 - Protein-protein interaction analysis. Protein-protein interaction networks of up-regulated genes in (A) C. burnetii-infected and (B) L. mexicana-infected THP1 cells visualized in STRING. Colors of circles are based on Markov Clustering with an inflation factor of 2. Highlighted clusters are labeled with their GO or KEGG categories. 


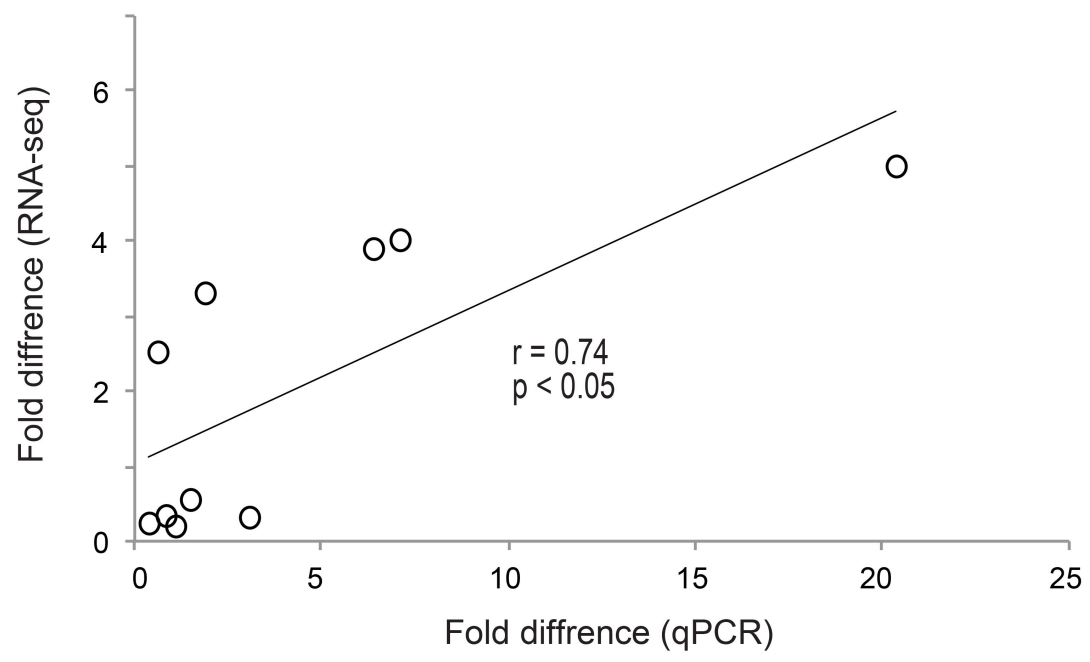

Figure 3.3 - Validation by qPCR of expression levels estimated by RNA-seq. Fold difference values calculated by qPCR correlated well with fold difference values calculated by RNA-seq. 
isoforms of p53 (encoded by TP53 gene) are involved in host defense against both bacterial (Helicobacter pylori) and viral (Influenza and Simian virus 40) infections (Terrier et al. 2013). Similarly, Hepatitis C virus activates the immunologic isoform of nitric oxide synthase (NOS) gene, which induces NO production (Machida et al. 2004). Transcriptome analysis (RNA-seq) is a powerful approach to identify differential isoform expression under different conditions at a genome-wide scale (Eswaran et al. 2013; Lo et al. 2014). We used RNA-seq to investigate whether infection by either Coxiella or Leishmania induced differential expression of human gene isoforms. We identified 689 isoforms from 626 genes that were differentially expressed in C. burnetii-infected cells, and 651 isoforms from 569 genes in Leishmania-infected cells, when compared to uninfected THP-1 cells (Figure 3.4, Additional_file_A5.csv, Additional_file_A6.csv). As observed for full-length mRNAs, there was minimal overlap between the sets of genes with differential expression of isoforms under each infection condition (only 105 common genes). Additionally, no KEGG pathways were significantly enriched in either gene set, indicating that differential isoform expression is a cell-wide phenomenon. Cumulatively, our data revealed that in addition to differences that are apparent at the gene level, the mostly unexplored realm of isoform variation could contribute to host responses to infections.

\section{Coxiella and Leishmania infections perturb the expression of apoptosis-related miRNAs}

Expression of various protein-coding genes in humans is regulated by miRNAs. These 
A

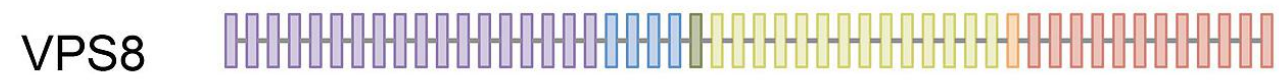

B

VPS8.1

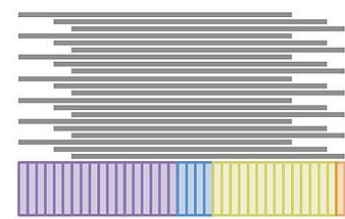

Coxiella-infected
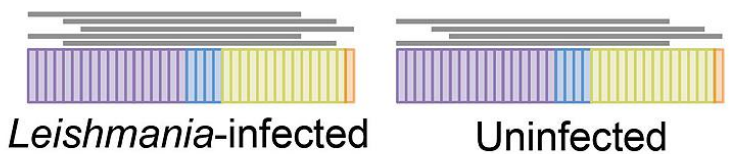

Uninfected

C

VPS8.7
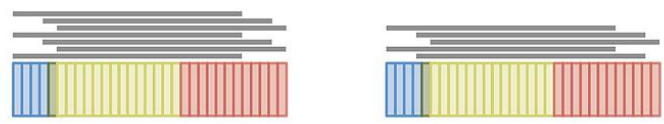

Coxiella-infected

Leishmania-infected

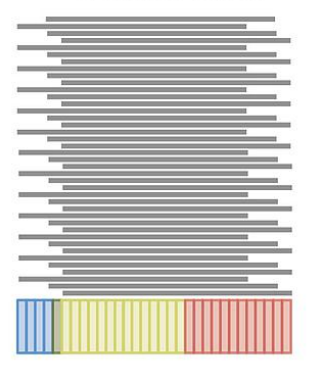

Uninfected

Figure 3.4 - Differential expression of mRNA isoforms. Differential isoform expression of VPS8 in C. burnetii infected, L. mexicana infected, and uninfected THP1 cells are shown as a representation of isoform analysis. (A) The full-length VPS8 gene is depicted with colored bars representing exons. Isoforms 1 (B) and 7 (C) of VPS8 that have significantly different expression in the three samples are shown. Each gray line above an isoform represents 10 mapped reads. 
small non-coding RNAs regulate the expression of target genes by base-pairing with mRNAs, thereby either blocking translation or causing target degradation or destabilization (Fabian et al. 2010). They are involved in many, if not all, biological processes, including metabolic pathways, cell proliferation and apoptosis. Recently, miRNAs have been shown to be an important part of host cell response to viral, bacterial and parasitic infections (Schulte et al. 2011; Schnitger et al. 2011; Lagos et al. 2010). In addition, some viruses, including Herpes viruses and Hepatitis $\mathrm{C}$ virus, have the ability to interfere with the host miRNA network to promote viral growth (Cullen 2011; Jopling et al. 2005). Recent studies also showed that eukaryotic intracellular pathogens such as Cryptosporidium parvum and Toxoplasma gondii promote intracellular replication by altering host cell miRNA networks (Hakimi and Ménard 2010; Zeiner et al. 2010). To identify miRNAs that are potentially perturbed by C. burnetii or L. mexicana infections, we sequenced and enumerated miRNAs expressed by uninfected, Coxiella-infected, and Leishmania-infected THP-1 cells. We identified 257 miRNAs that were expressed in THP-1 cells (Additional_file_A7.csv), which includes 50 of the 64 miRNAs reported by a recent study that examined miRNAs expressed in human macrophages in response to Leishmania major infection (Lemaire et al. 2013). Among the 257 miRNAs, seven were upregulated and one was down regulated in Coxiella-infected cells, and three were upregulated and two were down regulated in Leishmania-infected cells (Table 3.2). Intriguingly, several of the differentially expressed miRNAs have been shown in previous studies to regulate host cell death: miR-145 modulates the expression of KLF4 (DavisDusenbery et al. 2011), a transcription factor for TP53, which regulates apoptosis 
Table 3.2 - MicroRNAs (miRNAs) perturbed by Coxiella and Leishmania infections

\begin{tabular}{|c|c|c|c|c|c|}
\hline Sample & miRNA & $\begin{array}{l}\text { Fold } \\
\text { Change } \\
(\log 2)\end{array}$ & $P$ value & Regulation & Process \\
\hline \multirow[t]{8}{*}{ Coxiella-infected } & mir-148a-3p & -0.58 & 0.024 & Down & Pro-apoptotic $^{\mathrm{a}}$ \\
\hline & $\operatorname{mir}-181 d-5 p$ & 0.78 & $<0.001$ & Up & Anti-apoptotic $^{\mathrm{b}}$ \\
\hline & $\operatorname{mir}-193 a-5 p$ & 0.81 & $<0.001$ & Up & Pro-apoptotic ${ }^{c}$ \\
\hline & $\operatorname{mir}-362-5 p$ & 0.89 & 0.015 & Up & Anti-apoptotic ${ }^{\mathrm{d}}$ \\
\hline & $\operatorname{mir}-361-5 p$ & 0.95 & 0.004 & $\mathrm{Up}$ & Anti-apoptotic ${ }^{\mathrm{e}}$ \\
\hline & $\operatorname{mir}-194-2-5 p$ & 1.05 & 0.024 & Up & Anti-apoptotic ${ }^{f}$ \\
\hline & $\operatorname{mir}-28-3 p$ & 1.12 & 0.024 & Up & Neither ${ }^{\mathrm{g}}$ \\
\hline & $\operatorname{mir}-28-5 p$ & 1.35 & $<0.001$ & Up & Pro-apoptotic ${ }^{g}$ \\
\hline \multirow[t]{5}{*}{ Leishmania-infected } & $\operatorname{mir}-145-5 p$ & -1.00 & 0.002 & Down & Pro-apoptotic ${ }^{\mathrm{h}}$ \\
\hline & $\operatorname{mir}-221-5 p$ & -0.62 & $<0.001$ & Down & Anti-apoptotic ${ }^{\mathrm{i}}$ \\
\hline & $\operatorname{mir}-15 b-5 p$ & 0.56 & 0.035 & $\mathrm{Up}$ & Pro-apoptotic ${ }^{\mathrm{j}}$ \\
\hline & $\operatorname{mir}-29 b-1-3 p$ & 1.09 & 0.002 & Up & Pro-apoptotic ${ }^{\mathrm{k}}$ \\
\hline & $\operatorname{mir}-29 b-2-3 p$ & 1.19 & $<0.001$ & Up & Pro-apoptotic ${ }^{\mathrm{k}}$ \\
\hline
\end{tabular}

$\overline{\mathrm{a}}$ (Zhang et al., 2011), ${ }^{\mathrm{b}}$ (Wang et al., 2010), ${ }^{\mathrm{c}}$ (Nakano et al., 2013), ${ }^{\mathrm{d}}$ (Xia et al., 2014), ${ }^{\mathrm{e}}$ (Wu et al., 2013), ${ }^{\mathrm{f}}$ (Zhang et al., 2014), ${ }^{\mathrm{g}}$ (Almeida et al., 2012), ${ }^{\mathrm{h}}$ (Davis-Dusenbery et al., 2011), ${ }^{\mathrm{i}}$ (le Sage et al., 2007), ${ }^{\mathrm{j}}$ (Cimmino et al., 2005), ${ }^{\mathrm{k}}$ (Garzon et al., 2009) 
(Rowland et al. 2005); miR-15b and miR-29b are known to be pro-apoptotic in leukemia cells (Cimmino et al. 2005; Garzon et al. 2009); miR-148a promotes apoptosis by targeting BCL2 in colorectal cancer cells (Zhang et al. 2011); miR-181d also targets BCL-2 and promotes apoptosis in glioma cells (Wang et al. 2012). These results complement gene expression data (Figure 3.2), and indicate that miRNAs may have important roles in inhibiting host cell death during Coxiella infection, and promoting host cell death during Leishmania infection. 


\section{CHAPTER 4}

\section{Conclusion}

Coxiella's basic biology and molecular pathogenesis has remained elusive and generally understudied. There is an urgent need to expand research to fill this void and to develop more effective therapies. By examining several critical biosynthetic pathways, we show that horizontal gene transfer (HGT) has played an important role in shaping the intracellular metabolic capability of C. burnetii. We found that not only has Coxiella horizontally acquired extra copies of genes that enhance fatty acid, biotin and heme biosyntheses, but also the mechanism for increased heme production stems from a nontranslational function of $\mathrm{tRNA}^{\mathrm{Glu}} 2$. Combined with the finding that Coxiella's growth is significantly reduced when HemL protein (part of the heme biosynthesis pathway) is inhibited, we showed that heme biosynthesis is critical to Coxiella's intracellular growth, and importantly, because HemL is not found in humans, it could be a prime target for developing new therapeutics.

Due to the uncertainty in C. burnetii's phylogenetic relationship with other Gammaproteobacteria, we focused on a subset of genes that were likely acquired from distantly related bacteria. However, horizontal exchange usually occurs at higher frequency between closely related bacteria (Ochman et al. 2000). Hence, it is likely that many more genes in C. burnetii are of HGT origin from more closely related bacteria. Availability of more genomes of bacteria in the phylogenetic neighborhood of C. burnetii along with more detailed evolutionary, genetic, and functional analyses are required to 
identify all HGT-origin genes in C. burnetii. Then, we can begin to understand the full impact of HGT on the pathogen's physiology and pathogenicity.

At the host level, the genome-wide gene, mRNA-isoform, and miRNA expression patterns were distinct between macrophages infected with either C. burnetii or $L$. Mexicana. This pathogen-specific response indicates that even though both pathogens have converged on a similar intracellular niche, they utilize distinct programs to generate and maintain their respective intracellular vacuoles. Of particular interest were several miRNAs known to inhibit apoptosis in human cells that are induced or repressed during Coxiella infection. One such miRNA is miR-148a, which inhibits apoptosis by targeting BCL-2. The expression of miR-148a was significantly reduced in Coxiella-infected cells when compared to uninfected cells; concordantly, the expression of Bcl-2 was higher in Coxiella infected cells. Based on this, our working hypothesis is that miR-148a regulates Bcl-2 mRNA levels, thereby promoting Coxiella infection. This current study focused mainly on RNA-seq analysis, thus further experiments need to be conducted to confirm that Bcl-2 is indeed the target of miR-148a in THP-1 cells. Additional work will be required to assess the role of the individual miRNAs identified here in promoting Coxiella infection, and how these miRNAs can be targeted to develop new, more effective therapies. 


\section{REFERENCES}

Alix E, Mukherjee S, Roy CR. 2011. Subversion of membrane transport pathways by vacuolar pathogens. J Cell Biol 195: 943-952. doi: 10.1083/jcb.201105019.

Almeida M, Nicoloso M, Zeng L, Ivan C. 2012. Strand-specific miR-28-5p and miR-28$3 p$ have distinct effects in colorectal cancer cells. Gastroenterology 142: 886-896. doi: 10.1053/j.gastro.2011.12.047.Strand-Specific.

Almiron, M, Martinez M, Sanjuan N, Ugalde R. 2001. Ferrochelatase is present in Brucella abortus and is critical for its intracellular survival and virulence. Infect Immun 69: 6225-6230. doi: 10.1128/IAI.69.10.6225-6230.2001.

Anzaldi LL, Skaar EP. 2010. Overcoming the heme paradox: Heme toxicity and tolerance in bacterial pathogens. Infect Immun 78: 4977-4989. doi: 10.1128/IAI.00613-10.

Beare PA, Gilk SD, Larson CL, Hill J, Stead CM, Omsland A, Cockrell DC, Howe D, Voth DE, Heinzen RA. 2011. Dot/Icm type IVB secretion system requirements for Coxiella burnetii growth in human macrophages. MBio 2: 1-10. doi: 10.1128/mBio.00175-11.

Beare PA, Larson CL, Gilk SD, Heinzen RA. 2012. Two systems for targeted gene deletion in Coxiella burnetii. Appl Environ Microbiol 78: 4580-4589. doi: 10.1128/AEM.00881-12.

Beare PA, Unsworth N, Andoh M, Voth DE, Omsland A, Gilk SD, Williams KP, Sobral BW, Kupko JJ 3rd, Porcella SF, Samuel JE, Heinzen RA. 2009. Comparative 
genomics reveal extensive transposon-mediated genomic plasticity and diversity among potential effector proteins within the genus Coxiella. Infect Immun 77: 642-656. doi: 10.1128/IAI.01141-08.

Beare PA, Unsworth N, Andoh M, Voth DE, Omsland A, Gilk SD, Williams KP, Sobral BW, Kupko JJ 3rd, Porcella SF, Samuel JE, Heinzen RA. 2006. Genetic diversity of the $\mathrm{Q}$ fever agent, Coxiella burnetii, assessed by microarray-based wholegenome comparisons. J Bacteriol 188: 2309-2324. doi: 10.1128/JB.188.7.23092324.2006.

Bolger AM, Lohse M, Usadel B. 2014. Trimmomatic: A flexible trimmer for Illumina sequence data. Bioinformatics 30: 2114-2120. doi:

10.1093/bioinformatics/btu170.

Brohée S, van Helden J. 2006. Evaluation of clustering algorithms for protein-protein interaction networks. BMC Bioinformatics 7: 488. doi: 10.1186/1471-2105-7-488.

Chen C, Banga S, Mertens K, Weber MM, Gorbaslieva I, Tan Y, Luo ZQ, Samuel JE. 2010. Large-scale identification and translocation of type IV secretion substrates by Coxiella burnetii. Proc Natl Acad Sci 107: 21755-21760. doi: 10.1073/pnas.1010485107.

Cimmino A, Calin GA, Fabbri M, Iorio MV, Ferracin M, Shimizu M, Wojcik SE, Aqeilan RI, Zupo S, Dono M, Rassenti L, Alder H, Volinia S, Liu CG, Kipps TJ, Negrini M, Croce CM. 2005. miR-15 and miR-16 induce apoptosis by targeting BCL2. Proc Natl Acad Sci 102: 13944-13949. doi: 10.1073/pnas.0506654102. 
Contreras I, Estrada JA, Guak H, Martel C, Borjian A, Ralph B, Shio MT, Fournier S, Krawczyk CM, Olivier M. 2014. Impact of Leishmania mexicana infection on dendritic cell signaling and functions. PLoS Negl Trop Dis 8: e3202. doi: 10.1371/journal.pntd.0003202.

Cullen BR. 2011. Viruses and microRNAs: RISCy interactions with serious consequences. Genes Dev 25: 1881-94. doi: 10.1101/gad.17352611.plasmic.

Darriba D, Taboada GL, Doallo R, Posada D. 2012. jModelTest 2: More models, new heuristics and parallel computing. Nat Methods 9: 772. doi: 10.1038/nmeth.2109.

Davis-Dusenbery BN, Chan MC, Reno KE, Weisman AS, Layne MD, Lagna G, Hata A. 2011. Down-regulation of Kruppel-like factor-4 (KLF4) by microRNA-143/145 is critical for modulation of vascular smooth muscle cell phenotype by transforming growth factor-beta and bone morphogenetic protein 4. J Biol Chem 286: 2809728110. doi: 10.1074/jbc.M111.236950.

De Kok A, Hengeveld AF, Martin A, Westphal AH. 1998. The pyruvate dehydrogenase multi-enzyme complex from Gram-negative bacteria. Biochim Biophys Acta 1385: 353-366. doi: 10.1128/AEM.01706-10.

De Muylder G, Ang KKH, Chen S, Arkin MR., Engel JC., McKerrow JH. 2011. A screen against Leishmania intracellular amastigotes: Comparison to a promastigote screen and identification of a host cell-specific hit. PLoS Negl Trop Dis 5: e1253. doi: 10.1371/journal.pntd.0001253.

Duron O, Noël V, McCoy KD, Bonazzi M, Sidi-Boumedine K, Morel O, Vavre F, Zenner L, Jourdain E, Durand P, Arnathau C, Renaud F, Trape JF, Biguezoton 
AS, Cremaschi J, Dietrich M, Léger E, Appelgren A, Dupraz M, Gómez-Díaz E, Diatta G, Dayo GK, Adakal H, Zoungrana S, Vial L, Chevillon C. 2015. The recent evolution of a maternally-inherited endosymbiont of ticks led to the emergence of the Q Fever pathogen, Coxiella burnetii. PLoS Pathog 11: e1004892. doi: 10.1371/journal.ppat.1004892.

Eisen JA. 2000. Horizontal gene transfer among microbial genomes: new insights from complete genome analysis. Curr Opin Genet Dev 10: 606-611. doi: 10.1016/S0959-437X(00)00143-X.

Eswaran J, Horvath A, Godbole S, Reddy SD, Mudvari P, Ohshiro K, Cyanam D, Nair S, Fuqua SA, Polyak K, Florea LD, Kumar R. 2013. RNA sequencing of cancer reveals novel splicing alterations. Sci Rep 3: 1689. doi: 10.1038/srep01689.

Fabian MR, Sonenberg N, Filipowicz W. 2010. Regulation of mRNA translation and stability by microRNAs. Annu Rev Biochem 79: 351-379. doi: 10.1146/annurevbiochem-060308-103103.

Feng Y, Cronan JE. 2009. Escherichia coli unsaturated fatty acid synthesis: complex transcription of the $f a b A$ gene and in vivo identification of the essential reaction catalyzed by FabB. J Biol Chem 284: 29526-29535. doi:

10.1074/jbc.M109.023440.

Feng Y, Napier BA, Manandhar M, Henke SK, Weiss DS, Cronan JE. 2014. A Francisella virulence factor catalyses an essential reaction of biotin synthesis. Mol Microbiol 91: 300-314. doi: 10.1111/mmi.12460. 
Flannagan RS, Cosío G, Grinstein S. 2009. Antimicrobial mechanisms of phagocytes and bacterial evasion strategies. Nat Rev Microbiol 7: 355-366. doi: 10.1038/nrmicro2128.

Franceschini A, Szklarczyk D, Frankild S, Kuhn M, Simonovic M, Roth A, Lin J, Minguez P, Bork P, von Mering C, Jensen LJ. 2013. STRING v9.1: Proteinprotein interaction networks, with increased coverage and integration. Nucl Acids Res 41: D808-D815. doi: 10.1093/nar/gks1094.

Frankenberg N, Moser J, Jahn D. 2003. Bacterial heme biosynthesis and its biotechnological application. Appl Microbiol Biotechnol 63: 115-127. doi: 10.1007/s00253-003-1432-2.

Frost LS, Leplae R, Summers AO, Toussaint A. 2005. Mobile genetic elements: The agents of open source evolution. Nat Rev Microbiol 3: 722-732. doi: $10.1038 /$ nrmicro1235.

Garzon R, Heaphy CE, Havelange V, Fabbri M, Volinia S, Tsao T, Zanesi N, Kornblau SM, Marcucci G, Calin GA, Andreeff M, Croce CM. 2009. MicroRNA 29b functions in acute myeloid leukemia. Blood 114: 5331-5342. doi: 10.1182/blood2009-03-211938.

Gerhart JG, Moses AS, Raghavan R. 2016. A Francisella-like endosymbiont in the Gulf Coast tick evolved from a mammalian pathogen. Sci Rep 6: 33670. doi: $10.1038 /$ srep33670.

Gilk SD, Beare PA, Heinzen RA. 2010. Coxiella burnetii expresses a functional $\Delta 24$ sterol reductase. J Bacteriol 192: 6154-6159. doi: 10.1128/JB.00818-10. 
Gilk SD. 2012. Role of lipids in Coxiella burnetii infection. Adv Exp Med Biol 984: 199213. doi: 10.1007/978-94-007-4315-1_10.

Gottlieb Y, Lalzar I, Klasson L. 2015. Distinctive genome reduction rates revealed by genomic analyses of two Coxiella-like endosymbionts in ticks. Genome Biol Evol 7: 1779-1796. doi: 10.1093/gbe/evv108.

Graham JG, MacDonald LJ, Hussain SK, Sharma UM, Kurten RC, Voth DE. 2013. Virulent Coxiella burnetii pathotypes productively infect primary human alveolar macrophages. Cell Microbiol. 15:1012-1025. doi: 10.1111/cmi.12096.

Hakimi MA, Ménard R. 2010. Do apicomplexan parasites hijack the host cell microRNA pathway for their intracellular development? F1000 Biol Rep 2: 16-18. doi: $10.3410 / \mathrm{B} 2-42$.

Herwaldt BL. 1999. Leishmaniasis. Lancet 354: 1191-1199. doi: 10.1016/S01406736(98)10178-2.

Huang DW, Sherman BT, Lempicki RA 2009a. Systematic and integrative analysis of large gene lists using DAVID bioinformatics resources. Nat Protoc 4: 44-57. doi: 10.1038/nprot.2008.211.

Huang DW, Sherman BT, Lempicki RA. 2009b. Bioinformatics enrichment tools: Paths toward the comprehensive functional analysis of large gene lists. Nucl Acids Res 37: 1-13. doi: 10.1093/nar/gkn923.

Huelsenbeck JP, Ronquist F. 2001. MRBAYES: Bayesian inference of phylogenetic trees. Bioinformatics 17: 754-755. doi: 10.1093/bioinformatics/17.8.754. 
Jopling C, Yi M, Lancaster A, Lemon S, Samow P. 2005. Modulation of hepatitis C virus RNA abundance by a liver-specific MicroRNA. Science 309: 1577-1581. doi: 10.1126/science. 1113329 .

Kearse M, Moir R, Wilson A, Stones-Havas S, Cheung M, Sturrock S, Buxton S, Cooper A, Markowitz S, Duran C, Thierer T, Ashton B, Meintjes P, Drummond A. 2012. Geneious Basic: An integrated and extendable desktop software platform for the organization and analysis of sequence data. Bioinformatics 28: 1647-1649. doi: 10.1093/bioinformatics/bts199.

Kim D, Pertea G, Trapnell C, Pimentel H, Kelley R, Salzberg SL. 2013. TopHat2: Accurate alignment of transcriptomes in the presence of insertions, deletions and gene fusions. Genome Biol 14: R36. doi: 10.1186/gb-2013-14-4-r36.

Kinchen JM, Ravichandran KS. 2008. Phagosome maturation: Going through the acid test. Nat Rev Mol Cell Biol 9: 781-795. doi: 10.1038/nrm2515.

Koski LB, Golding GB. 2001. The closest BLAST hit is often not the nearest neighbor. $J$ Mol Evol 52: 540-542. doi: 10.1007/s002390010184.

Kozomara A, Griffiths-Jones S. 2014. miRBase: Annotating high confidence microRNAs using deep sequencing data. Nucl Acids Res 42: D68-D73. doi: 10.1093/nar/gkt1181.

Lagos D, Pollara G, Henderson S, Gratrix F, Fabani M, Milne RS, Gotch F, Boshoff C. 2010. miR-132 regulates antiviral innate immunity through suppression of the p300 transcriptional co-activator. Nat Cell Biol 12: 513-519. doi: 10.1038/ncb2054. 
Lareau LF, Green RE, Bhatnagar RS, Brenner SE. 2004. The evolving roles of alternative splicing. Curr Opin Struct Biol 14: 273-282. doi: 10.1016/j.sbi.2004.05.002.

Lawrence JG, and Ochman H. 1997. Amelioration of bacterial genomes: Rates of change and exchange. J Mol Evol 44: 383-397. doi: 10.1007/PL00006158.

Lemaire J, Mkannez G, Guerfali FZ, Gustin C, Attia H, Sghaier RM; Sysco-Consortium, Dellagi K, Laouini D, Renard P. 2013. MicroRNA expression profile in human macrophages in response to Leishmania major infection. PLoS Negl Trop Dis 7: e2478. doi: 10.1371/journal.pntd.0002478.

Leng N, Dawson JA, Thomson JA, Ruotti V, Rissman AI, Smits BM, Haag JD, Gould MN, Stewart RM, Kendziorski C. 2013. EBSeq: An empirical Bayes hierarchical model for inference in RNA-seq experiments. Bioinformatics 29: 1035-1043. doi: 10.1093/bioinformatics/btt087.

le Sage C, Nagel R, Egan DA, Schrier M, Mesman E, Mangiola A, Anile C, Maira G, Mercatelli N, Ciafrè SA, Farace MG, Agami R. 2007. Regulation of the p27 (Kip1) tumor suppressor by miR-221 and miR-222 promotes cancer cell proliferation. EMBO J 26: 3699-3708. doi: 10.1038/sj.emboj.7601790.

Lerat E, Daubin V, Ochman H, Moran NA. 2005. Evolutionary origins of genomic repertoires in bacteria. PLoS Biol 3: e130. doi: 10.1371/journal.pbio.0030130.

Li H, Durbin R. 2010. Fast and accurate long-read alignment with Burrows-Wheeler transform. Bioinformatics 26: 589-595. doi: 10.1093/bioinformatics/btp698.

Lin S, Hanson RE, Cronan JE. 2010. Biotin synthesis begins by hijacking the fatty acid synthetic pathway. Nat Chem Biol 6: 682-688. doi: 10.1038/nchembio.420. 
Livak KJ, Schmittgen TD. 2001. Analysis of relative gene expression data using realtime quantitative PCR and the 2(-Delta Delta C(T)) Methods. 25: 402-408. doi: 10.1006/meth.2001.1262.

Lo WS, Gardiner E, Xu Z, Lau CF, Wang F, Zhou JJ, Mendlein JD, Nangle LA, Chiang KP, Yang XL, Au KF, Wong WH, Guo M, Zhang M, Schimmel P. 2014. Human tRNA synthetase catalytic nulls with diverse functions. Science 345: 328-332. doi: 10.1126/science.1252943.

Lo WS, Gasparich GE, Kuo CH. 2015. Found and lost: The fates of horizontally acquired genes in arthropod-symbiotic Spiroplasma. Genome Biol Evol 7: 2458-2472. doi: $10.1093 / \mathrm{gbe} / \mathrm{evv} 160$.

Lührmann A, Roy CR. 2007. Coxiella burnetii inhibits activation of host cell apoptosis through a mechanism that involves preventing cytochrome c release from mitochondria. Infect Immun 75: 5282-5289. doi: 10.1128/IAI.00863-07.

Machida K, Cheng KTH, Sung VMH, Lee KJ, Levine AM, Lai MMC. 2004. Hepatitis C virus infection activates the immunologic (type II) isoform of nitric oxide synthase and thereby enhances DNA damage and mutations of cellular genes. $J$ Virol 78: 8835-8843. doi: 10.1128/JVI.78.16.8835-8843.2004.

Mahapatra S, Ayoubi P, Shaw EI. 2010. Coxiella burnetii Nine Mile II proteins modulate gene expression of monocytic host cells during infection. BMC Microbiol 10: 244. doi: 10.1186/1471-2180-10-244. 
Martinez E, Cantet F, Bonazzi M. 2015. Generation and multi-phenotypic high-content screening of Coxiella burnetii transposon mutants. J Vis Exp e52851. doi: $10.3791 / 52851$.

Martinez E, Cantet F, Fava L, Norville I, Bonazzi M. 2014. Identification of OmpA, a Coxiella burnetii protein involved in host cell invasion, by multi-phenotypic highcontent screening. PLoS Pathog 10: e1004013. doi: 10.1371/journal.ppat.1004013.

Maurin M, Raoult D. 1999. Q fever. Clin Microbiol Rev 12: 518-553.

McConville MJ, de Souza D, Saunders E, Likic VA, Naderer T. 2007. Living in a phagolysosome; metabolism of Leishmania amastigotes. Trends Parasitol 23: 368-375. doi: 10.1016/j.pt.2007.06.009.

McCutcheon JP, Moran NA. 2012. Extreme genome reduction in symbiotic bacteria. Nat Rev Microbiol 10: 13-26. doi: 10.1038/nrmicro2670.

Mike LA, Dutter BF, Stauff DL, Moore JL, Vitko NP, Aranmolate O, Kehl-Fie TE, Sullivan S, Reid PR, DuBois JL, Richardson AR, Caprioli RM, Sulikowski GA, Skaar EP. 2013. Activation of heme biosynthesis by a small molecule that is toxic to fermenting Staphylococcus aureus. Proc Natl Acad Sci 110: 8206-8211. doi: 10.1073/pnas.1303674110.

Mira A, Ochman H, Moran NA. 2001. Deletional bias and the evolution of bacterial genomes. Trends Genet 17: 589-596. doi: 10.1016/S0168-9525(01)02447-7.

Moran NA. 2002. Microbial minimalism: Genome reduction in bacterial pathogens. Cell 108: 583-586. doi: 10.1016/S0092-8674(02)00665-7. 
Nakano H, Yamada Y, Miyazawa T, Yoshida T. 2013. Gain-of-function microRNA screens identify miR-193a regulating proliferation and apoptosis in epithelial ovarian cancer cells. Int J Oncol 42: 1875-1882. doi: 10.3892/ijo.2013.1896.

Narasaki CT, Toman R. 2012. Lipopolysaccharide of Coxiella burnetii. Adv Exp Med Biol 984: 65-90. doi: 10.1007/978-94-007-4315-1_4.

Nelson K, Selander RK. 1994. Intergeneric transfer and recombination of the 6phosphogluconate dehydrogenase gene (gnd) in enteric bacteria. Proc Natl Acad Sci 91: 10227-10231.

Newton HJ, Roy CR. 2011. The Coxiella burnetii Dot/Icm system creates a comfortable home through lysosomal renovation. MBio 2: e00226-e002211. doi: 10.1128/mBio.00226-11.

Ochman H, Davalos LM. 2006. The nature and dynamics of bacterial genomes. Science 311: 1730-1733. doi: 10.1126/science.1119966.

Ochman H, Lawrence JG, Groisman EA. 2000. Lateral gene transfer and the nature of bacterial innovation. Nature 405: 299-304. doi: 10.1038/35012500.

Ochman H, Moran NA. 2001. Genes lost and genes found: Evolution of bacterial pathogenesis and symbiosis. Science 292: 1096-1099. doi:

10.1126/science.1058543.

Olivier M, Gregory DJ, Forget G. 2005. Subversion mechanisms by which Leishmania parasites can escape the host immune response: A signaling point of view. Clin Microbiol Rev 18: 293-305. doi: 10.1128/CMR.18.2.293-305.2005. 
Omsland A, Beare PA, Hill J, Cockrell DC, Howe D, Hansen B, Samuel JE, Heinzen RA. 2011. Isolation from animal tissue and genetic transformation of Coxiella burnetii are facilitated by an improved axenic growth medium. Appl Environ Microbiol 77: 3720-3725. doi: 10.1128/AEM.02826-10.

Price MN, Dehal PS, Arkin AP. 2008. Horizontal gene transfer and the evolution of transcriptional regulation in Escherichia coli. Genome Biol 9: R4. doi: 10.1186/gb-2008-9-1-r4.

Rabhi I, Rabhi S, Ben-Othman R, Aniba MR; Sysco Consortium, Trentin B, Piquemal D, Regnault B, Guizani-Tabbane L. 2013. Comparative analysis of resistant and susceptible macrophage gene expression response to Leishmania major parasite. BMC Genomics 14: 723. doi: 10.1186/1471-2164-14-723.

Rabhi I, Rabhi S, Ben-Othman R, Rasche A, Daskalaki A, Trentin B, Piquemal D, Regnault B, Descoteaux A, Guizani-Tabbane L. 2012. Transcriptomic signature of Leishmania infected mice macrophages: A metabolic point of view. PLoS Negl Trop Dis 6: e1763. doi: 10.1371/journal.pntd.0001763.

Rabinovitch M, Veras PST. 1996. Cohabitation of Leishmania amazonensis and Coxiella burnetii. Trends Microbiol 4: 158-161. doi: 10.1016/0966-842X(96)10027-5.

Raghavan R, Hicks LD, Minnick MF. 2008. Toxic introns and parasitic intein in Coxiella burnetii: Legacies of a promiscuous past. J Bacteriol 190: 5934-5943. doi: 10.1128/JB.00602-08.

Raghavan R, Miller SR, Hicks LD, Minnick MF. 2007. The unusual 23S rRNA gene of Coxiella burnetii: Two self-splicing group I introns flank a 34-base-pair exon, and 
one element lacks the canonical omegaG. J Bacteriol 189: 6572-6579. doi: 10.1128/JB.00812-07.

Raghavan R, Sloan DB, Ochman H. 2012. Antisense transcription is pervasive but rarely conserved in enteric bacteria. MBio 3: e00156-e01512. doi:

10.1128/mBio.00156-12.

Real F, Florentino PT, Reis LC, Ramos-Sanchez EM, Veras PS, Goto H, Mortara RA. 2014. Cell-to-cell transfer of Leishmania amazonensis amastigotes is mediated by immunomodulatory LAMP-rich parasitophorous extrusions. Cell Microbiol 16: 1549-1564. doi: 10.1111/cmi.12311.

Real F, Mortara RA, Rabinovitch M. 2010. Fusion between Leishmania amazonensis and Leishmania major parasitophorous vacuoles: Live imaging of coinfected macrophages. PLoS Negl Trop Dis 4: e905. doi: 10.1371/journal.pntd.0000905.

Ren Q, Robertson S, Howe D, Barrows L, Heinzen R. 2003. Comparative DNA microarray analysis of host cell transcriptional responses to infection by Coxiella burnetii or Chlamydia trachomatis. Ann NY Acad Sci 990: 701-713. doi: 10.1111/j.1749-6632.2003.tb07447.x.

Rowland BD, Bernards R, Peeper DS. 2005. The KLF4 tumour suppressor is a transcriptional repressor of p53 that acts as a context-dependent oncogene. Nat Cell Biol 7: 1074-1082. doi: 10.1038/ncb1314.

Schnitger AK, Machova A, Mueller RU, Androulidaki A, Schermer B, Pasparakis M, Krönke M, Papadopoulou N. 2011. Listeria monocytogenes infection in 
macrophages induces vacuolar-dependent host miRNA response. PLoS One 6: e27435. doi: 10.1371/journal.pone.0027435.

Schulte LN, Eulalio A, Mollenkopf HJ, Reinhardt R, Vogel J. 2011. Analysis of the host microRNA response to Salmonella uncovers the control of major cytokines by the let-7 family. EMBO J 30: 1977-1989. doi: 10.1038/emboj.2011.94.

Seshadri R, Paulsen IT, Eisen JA, Read TD, Nelson KE, Nelson WC, Ward NL, Tettelin H, Davidsen TM, Beanan MJ, Deboy RT, Daugherty SC, Brinkac LM, Madupu R, Dodson RJ, Khouri HM, Lee KH, Carty HA, Scanlan D, Heinzen RA, Thompson HA, Samuel JE, Fraser CM, Heidelberg JF. 2003. Complete genome sequence of the Q-fever pathogen Coxiella burnetii. Proc Natl Acad Sci 100: 5455-5460. doi: 10.1073/pnas.0931379100.

Shannon JG, Howe D, Heinzen RA. 2005. Virulent Coxiella burnetii does not activate human dendritic cells: Role of lipopolysaccharide as a shielding molecule. Proc Natl Acad Sci 102: 8722-8727. doi: 10.1073/pnas.0501863102.

Sievers F, Wilm A, Dineen D, Gibson TJ, Karplus K, Li W, Lopez R, McWilliam H, Remmert M, Soding J, Thompson JD, Higgins DG. 2011. Fast, scalable generation of high-quality protein multiple sequence alignments using Clustal Omega. Mol Syst Biol 7: 539. doi: 10.1038/msb.2011.75.

Smith MG, Gianoulis TA, Pukatzki S, Mekalanos JJ, Ornston LN, Gerstein M, Snyder M. 2007. New insights into Acinetobacter baumannii pathogenesis revealed by highdensity pyrosequencing and transposon mutagenesis. Genes Dev 21: 601-614. doi: 10.1101/gad.1510307. 
Smith TA, Driscoll T, Gillespie JJ, Raghavan R. 2015. A Coxiella-like endosymbiont is a potential vitamin source for the Lone Star Tick. Genome Biol Evol 7: 831-838. doi: 10.1093/gbe/evv016.

Stamatakis A, Hoover P, Rougemont J. 2008. A rapid bootstrap algorithm for the RAxML web servers. Syst Biol 57: 758-771. doi: 10.1080/10635150802429642.

Swanson MS, Fernandez-Moreia E. 2002. A microbial strategy to multiply in macrophages: The pregnant pause. Traffic 3: 170-177. doi: 10.1034/j.16000854.2002.030302.x.

Talavera G, Castresana J. 2007. Improvement of phylogenies after removing divergent and ambiguously aligned blocks from protein sequence alignments. Syst Biol 56: 564-577. doi: 10.1080/10635150701472164.

Terrier O, Bourdon JC, Rosa-Calatrava M. 2013. P53 protein isoforms: Key regulators in the front line of pathogen infections? PLoS Pathog 9: e1003246. doi: 10.1371/journal.ppat.1003246.

Thomas CM, Nielsen KM. 2005. Mechanisms of, and barriers to, horizontal gene transfer between bacteria. Nat Rev Microbiol 3: 711-721. doi: 10.1038/nrmicro1234.

Treangen TJ, Rocha EP. 2011. Horizontal transfer, not duplication, drives the expansion of protein families in prokaryotes. PLoS Genet 7: e1001284. doi: 10.1371/journal.pgen.1001284.

Van Schaik EJ, Chen C, Mertens K, Weber MM, Samuel JE. 2013. Molecular pathogenesis of the obligate intracellular bacterium Coxiella burnetii. Nat Rev Microbiol 11: 561-573. doi: 10.1038/nrmicro3049. 
Veras PST, Moulia C, Dauguet C, Tunis CT, Thibon M, Rabinovitch M. 1995. Entry and survival of Leishmania amazonensis amastigotes within phagolysosome-like vacuoles that shelter Coxiella burnetii in Chinese hamster ovary cells. Infect Immun 63: 3502-3506.

Voth DE, Beare PA, Howe D, Sharma UM, Samoilis G, Cockrell DC, Omsland A, Heinzen RA. 2013. The Coxiella burnetii cryptic plasmid is enriched in genes encoding type IV secretion system substrates. J Bacteriol 193: 1493-1503. doi: 10.1128/JB.01359-10.

Voth DE, Heinzen RA. 2007. Lounging in a lysosome: The intracellular lifestyle of Coxiella burnetii. Cell Microbiol 9: 829-840. doi: 10.1111/j.14625822.2007.00901.x.

Voth DE, Howe D, Heinzen RA. 2007. Coxiella burnetii inhibits apoptosis in human THP-1 cells and monkey primary alveolar macrophages. Infect Immun 75: 42634271. doi: 10.1128/IAI.00594-07.

Wakasugi K, Slike BM, Hood J, Otani A, Ewalt KL, Friedlander M, Cheresh DA, Schimmel P. 2002. A human aminoacyl-tRNA synthetase as a regulator of angiogenesis. Proc Natl Acad Sci 99: 173-177. doi: 10.1073/pnas.012602099.

Waller RF, McConville MJ. 2002. Developmental changes in lysosome morphology and function Leishmania parasites. Int J Parasitol. 32: 1435-1445. doi: 10.1016/S0020-7519(02)00140-6.

Wang LY, Brown L, Elliott M, Elliott T. 1997. Regulation of heme biosynthesis in Salmonella typhimurium: Activity of glutamyl-tRNA reductase (HemA) is greatly 
elevated during heme limitation by a mechanism which increases abundance of the protein. J Bacteriol 179: 2907-2914. doi: 10.1128/jb.179.9.2907-2914.1997.

Wang B, Hsu SH, Majumder S, Kutay H, Huang W, Jacob ST, Ghoshal K. 2010. TGFbeta-mediated upregulation of hepatic miR-181b promotes hepatocarcinogenesis by targeting TIMP3. Oncogene 29: 1787-1797. doi: 10.1038/onc.2009.468.

Wang XF, Shi ZM, Wang XR, Cao L, Wang YY, Zhang JX, Yin Y, Luo H, Kang CS, Liu N, Jiang T, You YP. 2012. MiR-181d acts as a tumor suppressor in glioma by targeting K-ras and Bcl-2. J Cancer Res Clin Oncol 138: 573-584. doi: 10.1007/s00432-011-1114-x.

Warrier I, Hicks LD, Battisti JM, Raghavan R, Minnick MF. 2014. Identification of novel small RNAs and characterization of the 6S RNA of Coxiella burnetii. PLoS ONE 9: e100147. doi: 10.1371/journal.pone.0100147.

Warrier I, Walter MC, Frangoulidis D, Raghavan R, Hicks LD, Minnick MF. 2016. The intervening sequence of Coxiella burnetii: Characterization and evolution. Front Cell Infect Microbiol 6: 83. doi: 10.3389/fcimb.2016.00083.

Williams KP, Gillespie JJ, Sobral BW, Nordberg EK, Snyder EE, Shallom JM, Dickerman AW. 2010. Phylogeny of gammaproteobacteria. J Bacteriol 192: 2305-2314. doi: 10.1128/JB.01480-09.

Woong Park S, Klotzsche M, Wilson DJ, Boshoff HI, Eoh H, Manjunatha U, Blumenthal A, Rhee K, Barry CE 3rd, Aldrich CC, Ehrt S, Schnappinger D. 2011. Evaluating the sensitivity of Mycobacterium tuberculosis to biotin deprivation using 
regulated gene expression. PLoS Pathog 7: e1002264. doi:

10.1371/journal.ppat.1002264.

Wu X, Xi X, Yan Q, Zhang Z, Cai B, Lu W, Wan X. 2013. MicroRNA-361-5p facilitates cervical cancer progression through mediation of epithelial-to-mesenchymal transition. Med Oncol 30: 751. doi: 10.1007/s12032-013-0751-0.

Xia JT, Chen LZ, Jian WH, Wang KB, Yang YZ, He WL, He YL, Chen D, Li W. 2014. MicroRNA-362 induces cell proliferation and apoptosis resistance in gastric cancer by activation of NF-kB signaling. $J$ Transl Med 12: 33. doi: 10.1186/14795876-12-33.

Zamboni DS, Campos MA, Torrecilhas AC, Kiss K, Samuel JE, Golenbock DT, Lauw FN, Roy CR, Almeida IC, Gazzinelli RT. 2004. Stimulation of toll-like receptor 2 by Coxiella burnetii is required for macrophage production of pro-inflammatory cytokines and resistance to infection. $J$ Biol Chem. 279: 54405-54415. doi: 10.1074/jbc.M410340200.

Zeiner GM, Norman KL, Thomson JM, Hammond SM, Boothroyd JC. 2010. Toxoplasma gondii infection specifically increases the levels of key host microRNAs. PLoS One 5: e8742. doi: 10.1371/journal.pone.0008742.

Zhang H, Li Y, Huang Q, Ren X, Hu H, Sheng H, Lai M. 2011. MiR-148a promotes apoptosis by targeting Bcl-2 in colorectal cancer. Cell Death Differ 18: 17021710. doi: $10.1038 /$ cdd.2011.28.

Zhang YM, Rock CO. 2008. Membrane lipid homeostasis in bacteria. Nat Rev Microbiol 6: 222-233. doi: 10.1038/nrmicro1839. 
Zhang J, Zhao CY, Zhang SH, Yu DH, Chen Y, Liu QH, Shi M, Ni CR, Zhu MH. 2014. Upregulation of miR-194 contributes to tumor growth and progression in pancreatic ductal adenocarcinoma. Oncol Rep 31: 1157-1164. doi: 10.3892/or.2013.2960.

Zhu Q, Kosoy M, Dittmar K. 2014. HGTector: An automated method facilitating genome-wide discovery of putative horizontal gene transfers. BMC Genomics 15: 717. doi: 10.1186/1471-2164-15-717.

Zlitni S, Ferruccio LF, Brown ED. 2013. Metabolic suppression identifies new antibacterial inhibitors under nutrient limitation. Nat Chem Biol 9: 796-804. doi: 10.1038/nchembio.1361. 


\section{APPENDIX A}

Additional_file_A1.csv - 172 'high-confidence' horizontally acquired genes on the chromosome of C. burnetii RSA 493 and their putative donors.

Additional_file_A2.csv - Differentially expressed genes in Coxiella-infected vs. uninfected THP-1 cells.

Additional_file_A3.csv - Differentially expressed genes in Leishmania-infected vs. uninfected THP-1 cells.

Additional_file_A4.csv - Differentially expressed genes that overlap between Coxiellainfected vs. uninfected and Leishmania-infected vs. uninfected THP-1 cells.

Additional_file_A5.csv - Differentially expressed mRNA isoforms in Coxiella-infected vs. uninfected THP-1 cells.

Additional_file_A6.csv - Differentially expressed mRNA isoforms in Leishmaniainfected vs. uninfected THP-1 cells.

Additional_file_A7.csv - All miRNAs detected in this study. 
Additional_file_A8.pdf - Supplementary tables and figures for Appendix B: “Genome rearrangements can make and break small RNA genes”.

Additional_file_A9.pdf - Supplementary tables and figures for Appendix C:

"Emergence of new sRNAs in enteric bacteria is associated with low expression and rapid evolution".

Additional_file_A10.csv - Table of 81 E. coli sRNAs and their descriptive statistics used in "Emergence of new sRNAs in enteric bacteria is associated with low expression and rapid evolution".

Additional_file_A11.csv - Table of 127 S. enterica sRNAs and their descriptive statistics used in "Emergence of new sRNAs in enteric bacteria is associated with low expression and rapid evolution".

Additional_file_A12.csv - Table of 49 E. coli rfam sRNAs and their descriptive statistics used in "Emergence of new sRNAs in enteric bacteria is associated with low expression and rapid evolution”.

Additional_file_A13.csv - Table of sRNAs conserved between E. coli and S. enterica and their descriptive statistics used in "Emergence of new sRNAs in enteric bacteria is associated with low expression and rapid evolution". 
Additional_file_A14.pdf - Supplementary tables and figures for Appendix D:

"Accumulation and expression of multiple antibiotic resistance genes in Arcobacter cryaerophilus that thrives in sewage".

Additional_file_A15.csv - Expression of ORFs mapped to antibiotic resistance categories

Additional_file_A16.csv - Comparison of overlapping genes and annotations in Arcobacter

Additional_file_A17.csv - Comparison of Arcobacter in RAST

Additional_file_A18.csv - Expression of ORFs mapped to virulence factors 


\section{APPENDIX B}

Genome rearrangements can make and break small RNA genes

\section{B.1 ABSTRACT}

Small RNAs (sRNAs) are short, transcribed regulatory elements that are typically encoded in the intergenic regions (IGRs) of bacterial genomes. Several sRNAs, first recognized in Escherichia coli, are conserved among enteric bacteria, but because of the regulatory roles of sRNAs, differences in sRNA repertoires might be responsible for features that differentiate closely related species. We scanned the E. coli MG1655 and Salmonella enterica Typhimurium genomes for nonsyntenic IGRs as a potential source of uncharacterized, species-specific sRNAs and found that genome rearrangements have reconfigured several IGRs causing the disruption and formation of sRNAs. Within an IGR that is present in E. coli but was disrupted in Salmonella by a translocation event is an sRNA that is associated with the FNR/CRP global regulators and influences $E$. coli biofilm formation. A Salmonella-specific sRNA evolved de novo through point mutations that generated a $\sigma^{70}$ promoter sequence in an IGR that arose through genome rearrangement events. The differences in the sRNA pools among bacterial species have previously been ascribed to duplication, deletion, or horizontal acquisition. Here, we show that genomic rearrangements also contribute to this process by either disrupting sRNA-containing IGRs or creating IGRs in which novel sRNAs may evolve. 


\section{B.2 INTRODUCTION}

RNAs that do not code for proteins are critical to gene regulation in all domains of life. In bacteria, small RNAs (sRNAs) are typically 50-200 nucleotides (nt) in length and are usually encoded in genomic regions between protein-coding genes (intergenic regions or IGRs). They can control gene expression by modulating transcription, translation, or mRNA stability (Storz et al. 2011). The application of technologies that interrogate entire transcriptomes has revealed unexpectedly large numbers of sRNAs in bacterial genomes (Raghavan, Groisman, et al. 2011; Kroger et al. 2012). But unlike protein-coding genes, the mechanisms by which new sRNA genes arise and the forces that shape the sRNAs contents of genomes are not well understood (Gottesman and Storz 2011). Some sRNAs, such as 6S RNA (Wassarman and Storz 2000), are broadly conserved among bacteria, whereas several others are species- or even strain-specific (Gottesman and Storz 2011; Skippington and Ragan 2012). The sRNA transcriptomes of the enterics Escherichia coli and Salmonella enterica show substantial overlap; however, some of the orthologous IGRs display different patterns of expression and several sRNAs are present in only one of the species (Raghavan et al. 2012).

Differences in sRNA gene contents among bacteria can arise from lineagespecific loss or from the emergence of new sRNAs through duplication (Lenz et al. 2004; Wilderman et al. 2004) or horizontal acquisition (Pichon and Felden 2005; Sittka et al. 2008). An examination of the distribution of sRNAs within the E. coli/ Shigella complex showed that the variation in the presence of known sRNAs was dominated by gene loss 
through deletions (Skippington and Ragan 2012). However, because this study focused only on those sRNAs that were originally characterized in a single strain of E. coli, it was biased toward the recognition of deletion events as it could not detect unique sRNAs in the genomes of other strains. Applying a broader phylogenetic perspective, homologs of a dual-function sRNA, SgrS, have been detected in distantly related Gammaproteobacteria (Horler and Vanderpool 2009), and an exhaustive survey of sRNAs revealed that most $E$. coli sRNAs originated after Enterobacteriales split from other Gammaproteobacteria (Peer and Margalit 2014). This lineage-specific sRNA accumulation seems to be related to the evolution of the RNA-binding protein Hfq; however, the mechanisms by which new bacterial sRNAs emerge or are lost remain largely unknown.

In eukaryotes, there are cases where novel regulatory RNAs have evolved through gene duplication, by de novo origination from noncoding sequences, and from the degradation of protein-coding genes (Kaessmann 2010); but in bacteria, the mechanisms by which new regulatory RNAs arise are much less clear. Because new genes can form through the chimeric assembly of fragments from various sources-one well-known example of this is the jingwei gene of Drosophila (Long and Langley 1993)—we first adopted a structural genomics and RNA sequencing (RNA-seq)-based approach to identify new sRNA genes and then tested for sRNA functions. This combination of comparative and experimental analyses identified several previously unrecognized sRNAs and uncovered the sources of these differences in sRNA repertoires. We find that genome rearrangements have disrupted and formed IGRs containing functional sRNAs, thereby causing disparity in the sRNA contents of related bacterial species. 


\section{B.3 MATERIALS AND METHODS}

\section{RNA Sequencing}

For sRNA discovery, E. coli K-12 MG1655 (GenBank NC_000913.2) and S. enterica subsp. enterica serovar Typhimurium str. 14028S (GenBank NC_016856.1) were grown in lysogeny broth $(\mathrm{LB})$ to $\mathrm{OD}_{600} \approx 0.5$ and then harvested by centrifugation. Total RNA was extracted from bacterial pellets using TRI reagent (Life Technologies), and cleaned on RNeasy columns (Qiagen) to remove spurious transcripts, transfer RNAs and 5S ribosomal RNA (rRNA). Genomic DNA was degraded by DNase treatment (Life Technologies) and 16S and 23S rRNAs were removed with a MICROBExpress kit (Life Technologies). Strand-specific RNA-seq libraries were synthesized (Raghavan et al. 2012), and each library was sequenced on the Illumina GA II platform (35 cycles) at the Yale Center for Genome Analysis.

\section{Mapping Sequencing Reads}

To identify sRNAs, sequencing reads were mapped onto the published E. coli (NC_000913.2) or Salmonella Typhimurium (NC_016856.1) genomes using MAQ (Li et al. 2008) and examined with Artemis (Rutherford et al. 2000), as described previously (Raghavan, Groisman, et al. 2011; Raghavan, Sage, et al. 2011; Raghavan et al. 2012). Those previously uncharacterized sRNAs identified in E. coli are numbered and given the prefix EcsR (E. coli sRNA) and those in Salmonella, SesR (S. enterica sRNA). 
To characterize regions that are differentially expression, sequencing reads were mapped onto E. coli (NC_000913.2) using the CLC Genomics Workbench. Genes with at least one read per sample and at least 20 total reads across all samples were chosen based on raw gene read counts from CLC mapping. Differential expression analysis of genes was performed using the DESeq R package (Anders and Huber 2010). Genes were chosen for downstream analysis based on significance $(P<0.05$, FDR-corrected). Gene Ontology (GO) terms were found using Database for Annotation, Visualization and Integrated Discovery (DAVID) and the GO FAT filter (Huang et al. 2009). GO-term enrichment tests were also performed with DAVID. GO-terms overrepresented among differentially expressed genes were chosen based on the level of statistical significance $(P<0.05$, Benjamini-corrected $)$.

\section{sRNA Target Identification}

To identify sRNA-regulated genes, EcsR1 was cloned into the NheI and HindIII sites behind the arabinose-inducible promoter on plasmid pBAD using the polymerase chain reaction (PCR) primers 5'-CCG CTA GCG TTT TAG TAT CCG CAT AAA GTG TAA C-3' and 5'-CTA AGC TTT CCT GCC CGC TGT TAT GGC G-3'. Escherichia coli or Salmonella, transformed with either the empty $\mathrm{pBAD}$ vector (control) or $\mathrm{pBAD}+$ EcsR1 (test), were grown in $\mathrm{LB}$ to $\mathrm{OD}_{600} \approx 0.5$ and induced with $0.2 \%$ arabinose for $15 \mathrm{~min}$, as previously described (Durand and Storz 2010). RNA was extracted and processed for Illumina sequencing as above. Four Illumina mRNA-seq libraries (two control samples and two test samples) were prepared for each bacterium and multiplexed into a single 
lane of an Illumina HiSeq 2000 (101 cycles) at the Genomic Sequencing and Analysis Facility at University of Texas at Austin.

\section{Measuring Hfq Stabilization of sRNAs}

An Hfq-deleted strain of E. coli (JW4130-1) and its isogenic parent strain (BW25113) (Baba et al. 2006) were obtained from Yale Coli Genetic Stock Center and grown to mid$\log$ phase $\left(\mathrm{OD}_{600} \approx 0.5\right)$ in LB. Total RNA was DNase-treated, and $1 \mu \mathrm{g}$ used as template for preparing cDNA. The abundances of EcsR1 in the wild-type and $H f q$-deleted strains were determined by quantitative PCR (primers: 5'-TTT TTG TGT AAT GAC GGA GTT CA-3', and 5'-GCG GGC TTT TTC TGC TTA TT-3'), and calculated from Ct (threshold cycle) values.

\section{Identification of Unique IGRs}

Orthologous genes common to E. coli and Salmonella were identified using a reciprocal BLAST best-hit approach (Raghavan et al. 2012). Gene order of each orthologous genepair was determined with GeneOrder 4.0 (Mahadevan and Seto 2010), and in cases where the genomic locations were not syntenic in the two species, we searched for gene-pairs with adjacent novel IGRs using Artemis.

\section{Identification of -10 Promoter Elements and sRNA Homologs}

Transcriptional start sites (TSSs) for novel sRNAs were identified from RNA-seq data as described previously (Raghavan, Sage, et al. 2011; Raghavan et al. 2012). TSSs for 
flanking genes were identified as above and were confirmed using published data (Kroger et al. 2012; Keseler et al. 2013). The $\sigma^{70}-10$ motif has a 6-bp consensus sequence TATAAT; however, promoters often have imperfect matches to the consensus and can be located anywhere in a window ranging from approximately 4 to $18 \mathrm{bp}$ upstream of the TSS (Huerta and Collado-Vides et al. 2003). To identify potential -10 elements associated with sRNAs, we searched this 15 -bp window for any hexamers that matched at least 4 of the $6 \mathrm{bp}$ in the consensus sequence including the two most highly conserved positions, A2 and T6 (Huerta et al. 2006). Bacterial genomes were queried for homologs of sRNAs identified in this study by analyzing a combination of sequence identity, secondary structure conservation, and genomic location as described previously (Raghavan, Groisman, et al. 2011).

\section{Detection of SRNA $3^{\prime}$-Ends}

A modified Rapid Amplification of cDNA Ends (RACE) procedure (Raghavan, Groisman, et al. 2011) was used to determine the $3^{\prime}$-ends of sRNAs as follows: Total RNA, depleted of 16S and 23S rRNA using a MICROBExpress kit (Life Technologies), was dephosphorylated with alkaline phosphatase (NEB), and a short oligonucleotide adapter (5'-P-UCG UAU GCC GUC UUC UGC UUG UidT-3') was ligated to 3'-ends using T4 RNA ligase (NEB). The 3' adapter-ligated RNA was reverse-transcribed using a primer complementary to the adapter (5'-CAA GCA GAA GAC GGC ATA CGA-3'), and the resulting cDNA was used as template in PCR reactions using primers specific to sRNAs (EcsR1: 5'-AGA TGA CAC TTT TTG TGT AAT GAC G-3'; EcsR2: 5'-TAT 
CGC GCT ACT TCA GGA TGA TGT A-3') along with the adapter-complementary primer. Amplicons were resolved on 3\% low-range ultra agarose (Bio-Rad) gels to determine their lengths, and their nucleotide sequences were determined by Sanger sequencing.

\section{Biofilm Assay}

EcsR1-deletion strain of $E$. coli was constructed using $\lambda$ Red-mediated recombination (Datsenko and Wanner 2000). Escherichia coli or Salmonella strains grown overnight at $37{ }^{\circ} \mathrm{C}$ in LB (or LB with $100 \mu \mathrm{g} / \mathrm{ml}$ ampicillin) were diluted $1: 100$ in fresh media and grown in 96-well microtiter plates for $48 \mathrm{~h}$ at $28{ }^{\circ} \mathrm{C}$ without shaking. Planktonic growth $\left(\mathrm{OD}_{600}\right)$ of E. coli and Salmonella strains measured on a Victor X5microplate reader (Perkin Elmer) did not significantly differ from each other. Supernatants containing nonadhered cells were discarded, and samples were washed twice with distilled water and the attached biofilm in each well was stained with $0.1 \%$ crystal violet for $30 \mathrm{~min}$. Unbound stain was removed by washing with distilled water. To quantify biofilm production, the crystal violet associated with biofilms was dissolved in $100 \%$ ethanol and absorbance $\left(\mathrm{A}_{600}\right)$ was measured, and normalized to the $\mathrm{OD}_{600}$ value of each strain, as described previously (Gualdi et al. 2008). Average intensity of biofilm formation for each strain was generated from at least four replicate experiments. 


\section{B.4 RESULTS}

\section{Genome Rearrangements Form Unique IGRs}

To identify IGRs that are unique to either E. coli or Salmonella, we compared the genomic locations of all orthologous genes in the two genomes. Because E. coli and Salmonella genomes are largely syntenic, the majority of IGRs situated between orthologous gene-pairs in the two genomes are also syntenic. However, there are several instances where orthologous protein-coding genes are situated at different relative locations in each genome (apparent as data points that do not lie along the diagonal in supplementary fig. S1, found in Additional_file_A8.pdf). After examining each of these cases, we identified chimeric IGRs present in either E. coli or Salmonella that were generated through the rearrangement of 68 genes (supplementary table S1, found in

\section{Additional_file_A8.pdf).}

\section{Unique IGRs Contain Novel sRNAs}

We performed a directional RNA-seq analysis on E. coli and Salmonella grown under identical conditions to determine whether any of the species-specific IGRs contained highly transcribed regions. After mapping sequencing reads onto the respective genomes, we detected "transcriptional peaks," which usually indicate the presence of sRNAs, in four of the species-specific IGRs, two in E. coli and two in Salmonella (Figure B.1). Transcripts mapping to the corresponding locations in the E. coli and Salmonella genomes have been observed in previous studies (Tjaden et al. 2002; Dornenburg et al. 
A

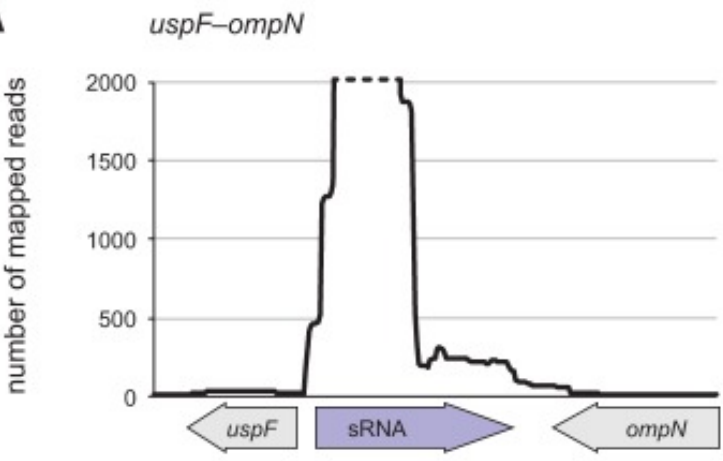

C

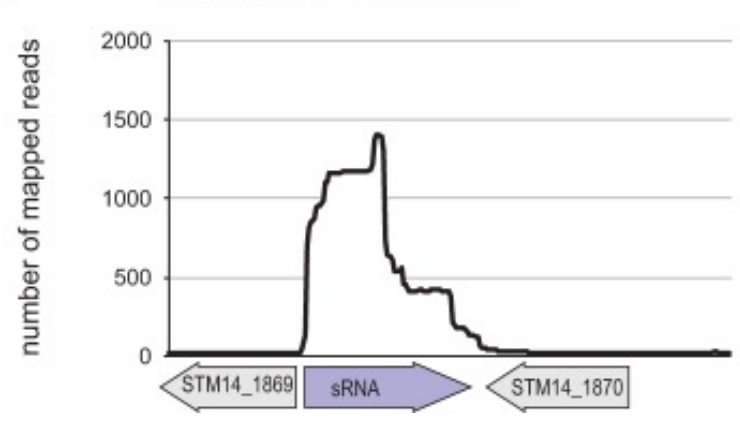

B $y a g u-y k g J$

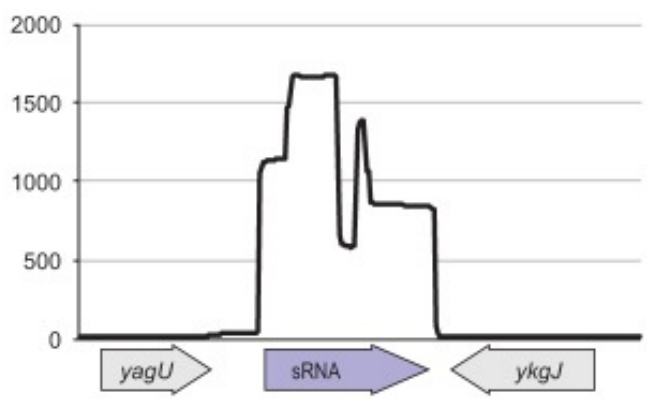

D STM14_1512-STM14_1513

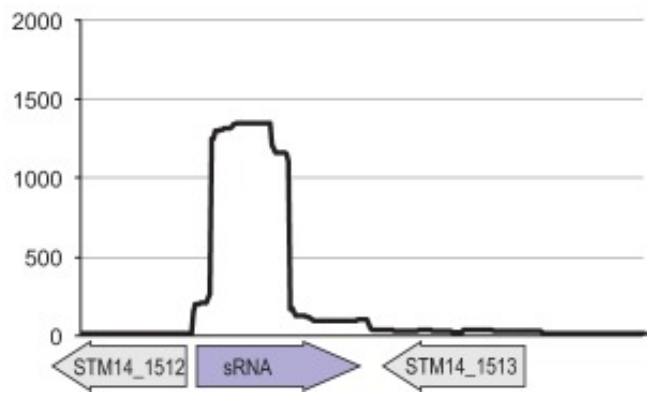

Figure B.1 - Expression profiles within nonsyntenic IGRs. Putative sRNAs were detected by RNA-seq analysis of transcript levels within IGRs in E. coli $(\mathbf{A}, \mathbf{B})$ and Salmonella (C, D). For uniformity, the number of sequencing reads mapped to IGRs is limited to 2,000 (dashed line). Arrows showing the orientation of ORFs and putative sRNAs are not drawn to scale. 
2010; Kroger et al. 2013) further verifying their transcriptional status, and there were no potential open reading frames (ORFs) of substantial length within these transcripts indicating that they represent sRNAs.

TSSs and 3'-ends of the transcribed sequences detected in these IGRs were identified from RNA-seq data (Raghavan et al. 2012), and a modified 3'-RACE procedure (Raghavan, Groisman, et al. 2011) was used to confirm the sRNAs in E. coli (supplementary fig. S2, found in Additional_file_A8.pdf), yielding the following results: 1) The sRNA (EcsR1) within the IGR between $u s p F$ and $o m p N$ genes in E. coli is $126 \mathrm{nt}$ (genomic location 1433654-1433779), 2) the sRNA (EcsR2) within the IGR between $y a g U$ and $y k g J$ genes in E. coli is $166 \mathrm{nt}$ (genomic location 302905-303070), 3) the Srna (SesR1) within the IGR between STM14_1512 and STM14_1513 genes in Salmonella is $105 \mathrm{nt}$ (genomic location 1347963-1348067), and 4) the sRNA (SesR2) within the IGR between STM14_1869 and STM14_1870 genes in Salmonella is $111 \mathrm{nt}$ (genomic location 1636380-1636490).

Homologs of EcsR1 and EcsR2 are present in all 66 E. coli genomes available in the RefSeq database (supplementary table S2, found in Additional_file_A8.pdf). SesR1 homologs were detected in all 44 S. enterica and S. bongori genomes (supplementary table S3, found in Additional_file_A8.pdf), and a recent study reported an sRNA (STnc1990) at the homologous position in S. enterica Typhimurium SL1344 (Kroger et al. 2013). SesR2 is conserved in 20 S. enterica genomes and in the two sequenced $S$. bongori strains (supplementary table S3, found in Additional_file_A8.pdf). However, the STM14_1869-STM14_1870 IGR is not maintained in S. bongori due to the loss of 
the STM14_1870 ortholog. Because SesR2 is absent from a few S. enterica serovars but present in the S. bongori outgroup, this sRNA is ancestral to Salmonella and was subsequently lost in some S. enterica lineages.

\section{A Salmonella-specific IGR Formed Through HGT-mediated Genome Rearrangement}

The STM14_1869-STM14_1870 IGR is present in Salmonella but not in E. coli. Escherichia coli possesses a gene, yjgH, that is orthologous to STM14_1869, but contained no ortholog for STM14_1870. Further analysis uncovered that STM14_1870 and its neighboring gene STM14_1871 constitute the toxin and antitoxin, respectively, of the StbED toxin-antitoxin (TA) system (Unterholzner et al. 2013) (Figure B.2).

Orthologs of $s t b E D$ TA genes are present on several enterobacterial plasmids and prophages, and are horizontally transferred between bacteria (Anantharaman and Aravind 2003; Unterholzner et al. 2013). Additionally, the succeeding gene in the Salmonella genome, STM14_1872, is also homologous to a gene of bacteriophage origin (Figure B.2), further indicating that the IGR between STM14_1869 and STM14_18670 was created by the introduction of genes through horizontal gene transfer (HGT)-mediated events.

\section{Evolution of a New sRNA in a Salmonella IGR}

To determine whether SesR2 was introduced along with its horizontally acquired neighboring genes into Salmonella, we searched the IGRs downstream of the $s t b E$ gene 
Salmonella Typhimurium str. 14028 S chromosome

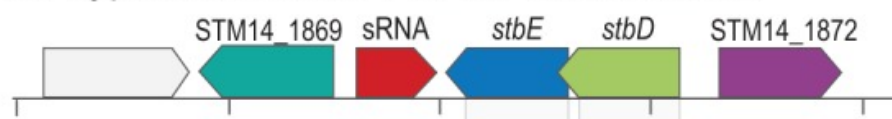

Erwinia pyrifoliae Ep1/96 plasmid pEP36

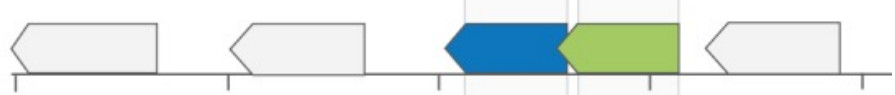

Klebsiella pneumoniae str. SB3432 chromosome

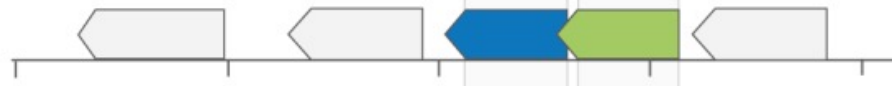

Citrobacter rodentium ICC168 plasmid pCROD2

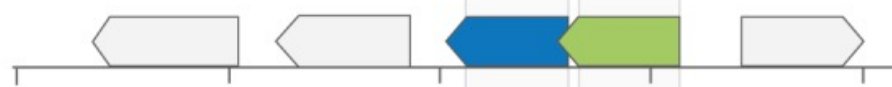

Shigella sonnei Ss046 plasmid pSS_046

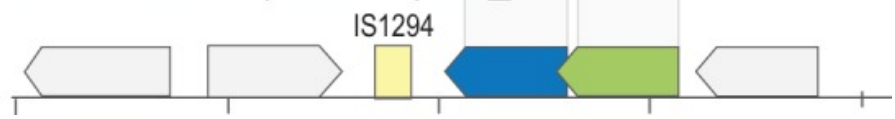

Figure B.2 - Salmonella IGR formed through an HGT-mediated genome

rearrangement. Most homologs of STM14_1870 (stbE, blue arrow) and STM14_1871 (stbD, green arrow) are situated on bacterial plasmids, and STM14_1872 (purple arrow) is a prophage gene. Both the STM14_1869-STM14_1870 IGR and the sRNA (SesR2) are present only in Salmonella. In Shigella, an insertion sequence (IS1294) flanks the $s t b E$ gene. 
in several enterobacterial genomes, but could not detect homologous sRNAs (Figure

B.2). Because a promoter is required for the new sRNA to be transcribed, we compared the region that contains the sRNA's TSS (5'-end of STM14_1869) with its homologous sequence in $E$. coli ( 5 '-end of $y j g H$ ) and in other enterics. As shown in Figure B.3 (and supplementary figure S3, found in Additional_file_A8.pdf), a putative $\sigma^{70}$ promoter (CATAAT, located -6 to -11 bp from sRNA's TSS) is uniquely present in Salmonella, indicating that this sRNA originated de novo in the Salmonella-specific IGR. Additionally, the promoter and sRNA sequences are conserved in S. bongori and in those S. enterica serovars that maintain an intact STM14_1869-STM14_1870 IGR.

\section{Rearrangement-Induced Loss of a Salmonella IGR}

In $E$. coli, the $o m p N$ and $u s p F$ genes are adjacent, separated by a 140-bp IGR that contains EcsR1; whereas in Salmonella, the $o m p N$ gene is in an alternate location, situated between the STM14_1775 and $r s t A$ genes. To determine the ancestry of these gene arrangements—specifically whether the $u s p F-o m p N$ IGR was gained by E. coli or lost by Salmonella —we analyzed the organization of the orthologous regions in the genomes of other enteric bacteria. The $u s p F-o m p N$ IGR is present and intact in other enteric species (Klebsiella pneumoniae, Enterobacter aerogenes, and Citrobacter koseri) establishing that this IGR predates the split between E. coli and Salmonella and was lost in Salmonella due to the relocation of $o m p N$ gene (Figure B.4, Figure B.5). As a consequence of this genome rearrangement in Salmonella, EcsR1 was split into two fragments located $\approx 200 \mathrm{~kb}$ apart, neither of which is transcribed (supplementary fig. 


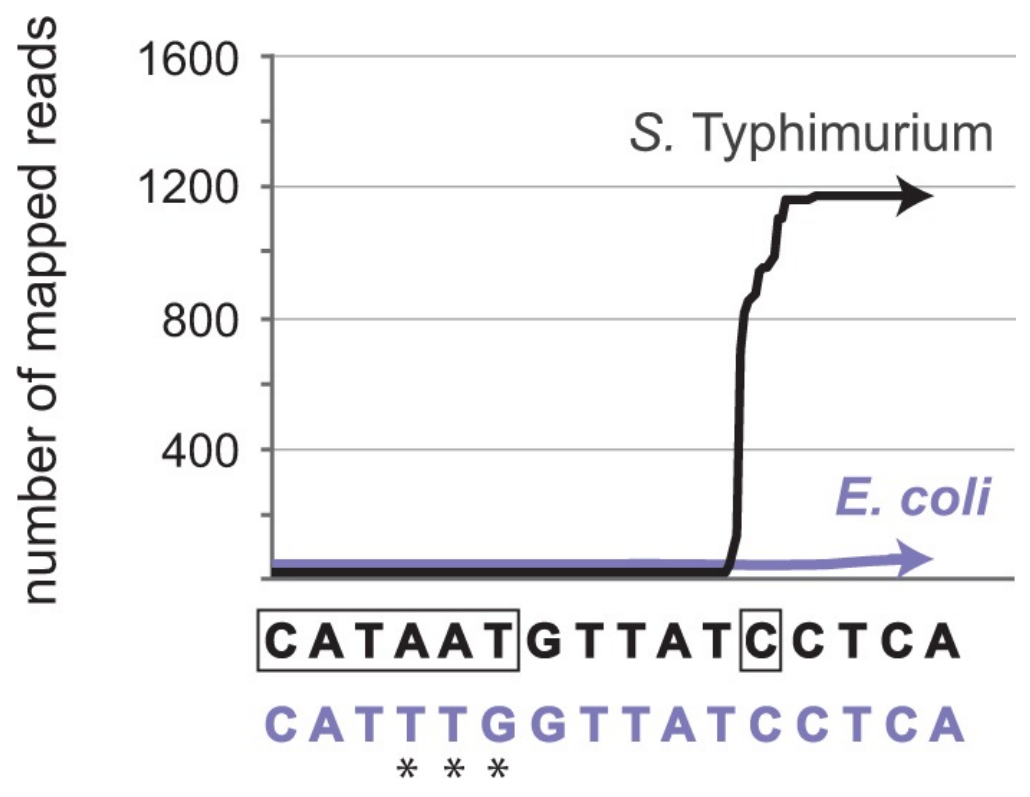

Figure B.3 - Evolution of a new sRNA promoter. Sequences immediately upstream of STM14_1869 (4471230-4471245) and its ortholog yjgH in E. coli (1636369-1636384) are aligned. Numbers of RNA-seq reads mapping to this region are shown (black, Salmonella; blue, E. coli). The new Salmonella $\sigma^{70}$ promoter and sRNA (SesR2) transcription start site are boxed. Asterisks indicate point mutations that differentiate the Salmonella sequence from the corresponding region in E. coli. 
A

Escherichia coli K-12 substr. MG1655

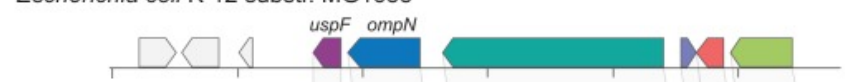

Citrobacter koseri ATCC BAA-895

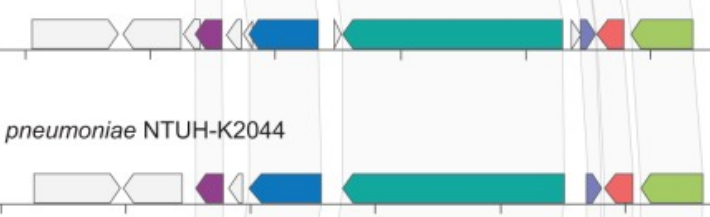

Enterobacter aerogenes KCTC 2190
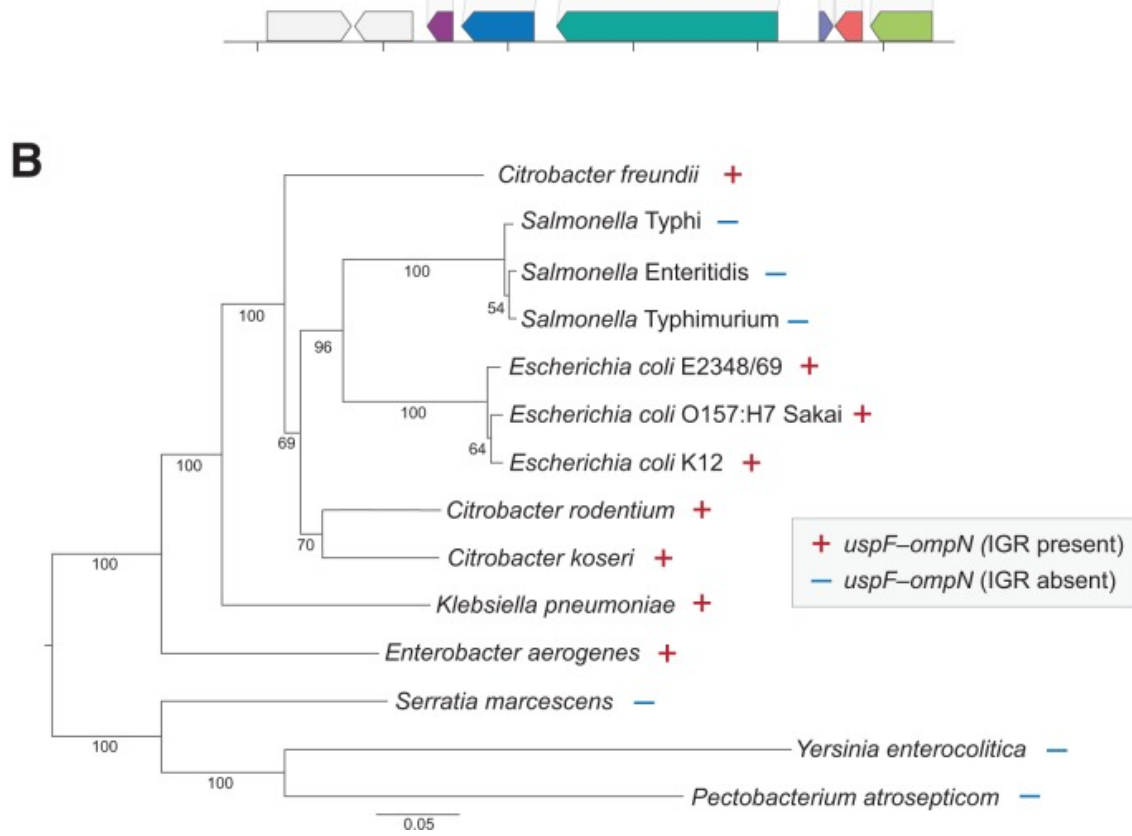

Figure B.4 - Distribution of the uspF-ompN IGR among enteric species. (A)

Alignment of genomic regions containing the $u s p F-o m p N$ IGR in $E$. coli and three other enteric species. Note that in both Citrobacter koseri and Klebsiella pneumoniae, small ORFs (gray arrows situated between $u s p F$ [purple] and $о m p N$ [blue]) have been predicted to occur in this IGR. (B) Phylogenetic tree (modified from Petty et al. 2010) showing the presence or absence of the $u s p F-o m p N$ IGR among species. 
E. coli

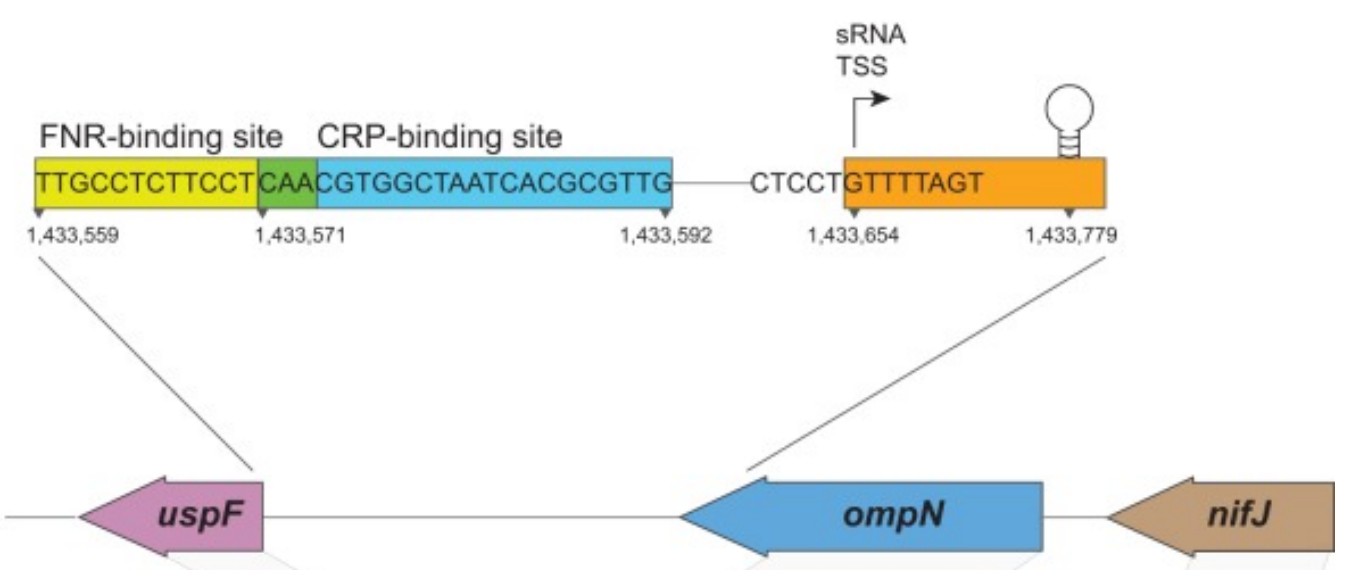

Salmonella

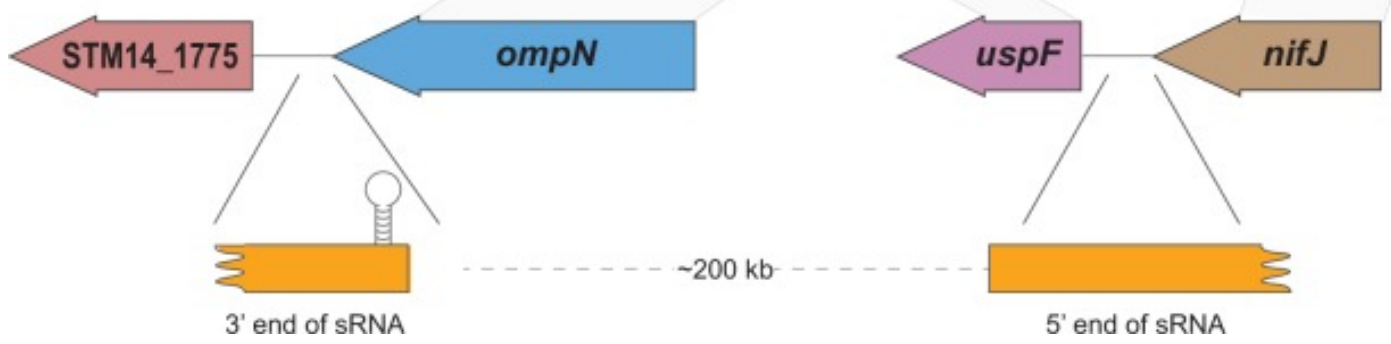

Figure B.5 - Loss of uspF-ompN IGR through genome rearrangement. The $u s p F-$ ompN IGR of E. coli was fragmented in Salmonella due to the translocation of $o m p N$ to a site adjacent to STM14_1775. The predicted FNR-and CRP-binding sites (yellow and blue, respectively; overlapping region in green) upstream of the sRNA (EcsR1) transcription start site (sRNA TSS) are shown. A predicted Rho-independent terminator (stem-loop structure) situated 3' of the sRNA is also depicted. 


\section{S4, found in Additional_file_A8.pdf).}

\section{EcsR1 Is Associated with Global Regulators in E. coli}

The $u s p F-o m p N$ IGR and EcsR1 are present in all strains of $E$. coli, which suggests that it maintains a regulatory function. To identify genes that are potentially under the control of EcsR1, we examined the effect of its overexpression on E. coli genes genome-wide, an approach that has been used previously to characterize the regulatory targets of sRNAs (Durand and Storz 2010; Beisel and Storz 2011). When analyzed by RNA-seq, the expression levels of 43 genes were significantly different $(P<0.05)$ in the EcsR1overexpressing strain when compared with wild-type E. coli (supplementary table S4, found in Additional_file_A8.pdf).A GO analysis uncovered bacterial membrane (GO:0031090), carbohydrate catabolic process (GO:0016052), and nitrate metabolic process (GO:0042126) as processes that were significantly enriched $(P<0.05)$ in our data set. Eleven downregulated genes were associated with these GO terms, of which nine were regulated by CRP and/or FNR (supplementary table S5, found in Additional_file_A8.pdf) (Constantinidou et al. 2006; Keseler et al. 2013). In concert with these observations, a 22-nt palindromic sequence with features resembling the consensus CRP-binding site and a putative 15-nt FNR-binding sequence (Figure B.5) were identified upstream of EcsR1, indicative of the sRNA being part of the CRP and FNR regulons. Expression of another E. coli sRNA, FnrS, is known to be affected by both FNR and CRP (Durand and Storz 2010), showing that CRP and FNR regulons overlap and may control multiple sRNAs. It has been shown previously that the 
transcriptional regulator CRP can control the expression of both an sRNA and the sRNA's target genes, and this "feed-forward loop" is thought to aid in the efficient modulation of gene expression in E. coli (Beisel and Storz 2011).

Because many sRNAs in E. coli associate with and are stabilized by the RNAbinding protein Hfq (De Lay et al. 2013), we examined whether Hfq stabilizes EcsR1. We measured the abundance of EcsR1 in wild-type and Hfq-deficient strains of E. coli, and found it to be significantly more abundant in the wild-type strain (supplementary fig. S5, found in Additional_file_A8.pdf), reinforcing its identity as an sRNA.

\section{EcsR1 Impacts Biofilm Formation}

Because CRP and FNR control carbohydrate and nitrate metabolism during biofilm formation (Van Alst et al. 2007; Karatan and Watnick 2009), we constructed EcsR1deletion and EcsR1-overexpression E. coli strains and measured the impact of this sRNA on biofilm formation. As shown in Figure B.6, biofilm production increased significantly $(P<0.0001)$ in the EcsR1-deleted strain when compared with wild-type E. coli.

Reintroduction of a plasmid-borne copy of EcsR1 into the deletion strain reduced biofilm formation to the same level as that of the wild-type strain (Figure B.6), indicating that biofilm-inhibition is an sRNA-specific phenotype.

\section{Expression of $E$. coli EcsR1 in Salmonella Activates Invasion-Associated Genes}

Biofilm production is important to virulence of enteric pathogens, so we tested the effects of EcsR1 on biofilm production in Salmonella by reintroducing the sRNA in an 


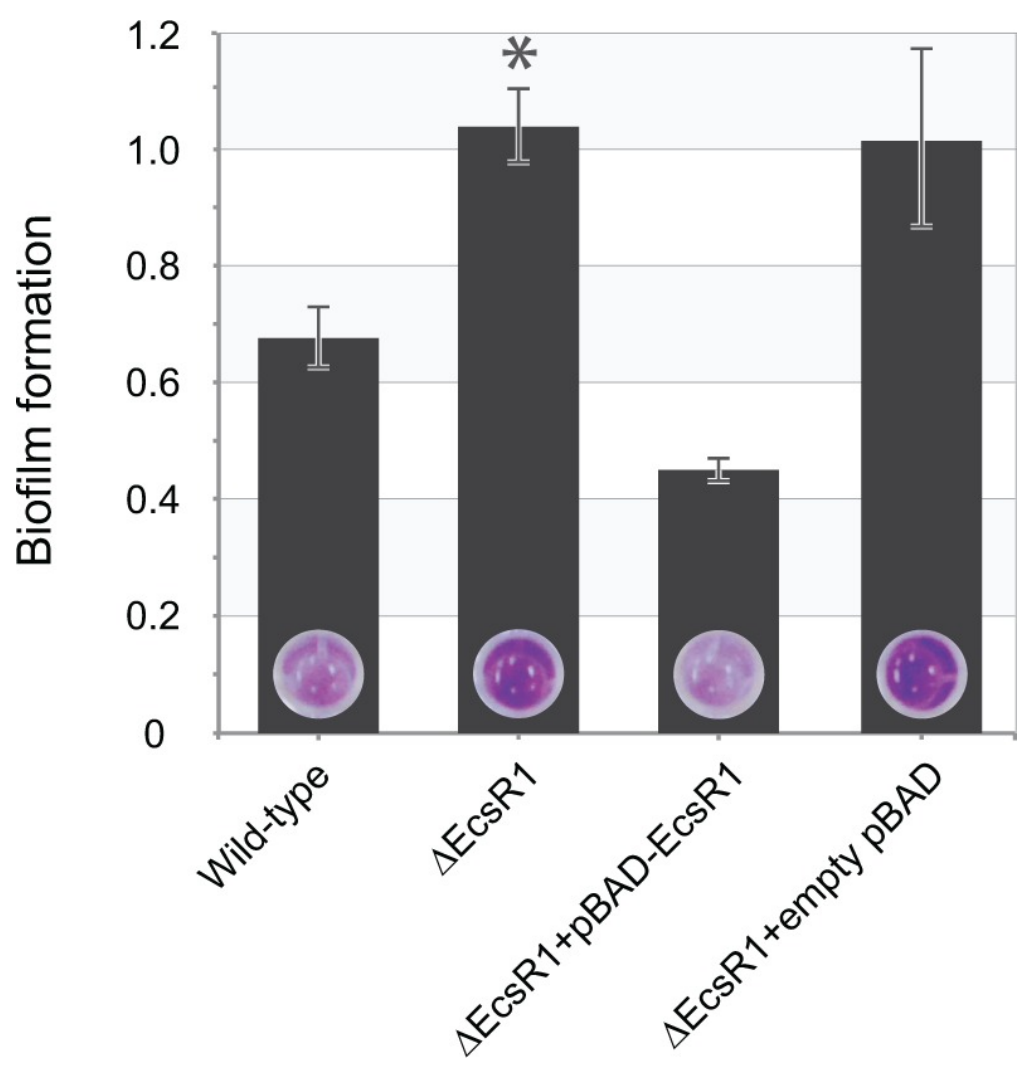

Figure B.6 - Biofilm formation is influenced by EcsR1. Escherichia coli biofilms stained with crystal violet were measured $\left(\mathrm{A}_{600}\right)$ after 48 -h growth at $28{ }^{\circ} \mathrm{C}$ and normalized to $\mathrm{OD}_{600}$ value. A wild-type strain, an EcsR1-deleted strain ( $\left.\Delta \mathrm{EcsR} 1\right)$, a $\Delta$ EcsR1 strain containing pBAD with cloned EcsR1 ( $\Delta$ EcsR1-pBAD-EcsR1), and a $\triangle$ EcsR1 strain containing empty pBAD ( $\triangle$ EcsR1-empty pBAD) were tested. Asterisks indicate a statistically significant difference between wild-type and $\Delta \mathrm{EcsR} 1$ strains $(P<$ $0.0001)$. 
expression vector. There was no significant difference in biofilm production between the wild-type and EcsR1-overexpression strains; the overexpression of EcsR1 in Salmonella alters the expression of 128 genes genome-wide (supplementary table S6, Supplementary Material online). GO analysis revealed nine processes (representing 27 genes) that were significantly enriched within this gene set, with "pathogenesis" (GO:0009405) being the most highly significant (supplementary table S7, found in Additional_file_A8.pdf). Among genes regulated by this sRNA, 22 are known to promote Salmonella invasion of host cells, most of which are situated within SPI-1 pathogenicity island (Fàbrega and Vila 2013). 


\section{B.5 DISCUSSION}

Our search for species-specific sRNAs was directed toward IGRs that were unique to either E. coli or Salmonella because most bacterial regulatory sRNAs are contained within these noncoding regions, although 3'-untranslated regions (UTRs) of mRNAs and promoters within mRNAs can also give rise to sRNAs (Chao et al. 2012; Guo et al. 2014). We found that genome rearrangements have altered IGRs and, in doing so, caused disparity in sRNA contents of these two species. A newly discovered sRNA (EcsR1), situated within the IGR between the $u s p F-o m p N$ genes in $E$. coli, is absent from Salmonella due to the translocation of a genomic segment containing the $o m p N$ gene. This sRNA is associated with the FNR and CRP regulons, and its expression impacts $E$. coli biofilm formation. Additionally, we identified an sRNA (SesR2) unique to Salmonella that evolved de novo in an IGR that was formed through a phage-mediated genome rearrangement. Although disparities in genome architectures are common among related species, these are the first known cases where rearrangements have caused the generation and destruction of sRNAs.

The main source of rearrangement events in bacterial genomes is homologous recombination across identical sequences. Escherichia coli and Salmonella contain numerous classes of repeat elements that can serve as templates for exchange (Rocha 2004). In addition, recombination between bacteriophage sequences in a genome can result in altered genome architectures in related bacteria (Brüssow et al. 2004); large proportions of both E. coli and Salmonella genomes consist of prophage genes (Bobay et 
al. 2013). Notwithstanding the large number of targets for homologous exchange, the gene order of E. coli and Salmonella has been well conserved despite an estimated 100Myr divergence (Ochman et al. 1999). The major difference in their genome architectures involves a large 600-kb inversion spanning the replication terminus and approximately 50 small-scale translocation events (supplementary fig. S1, found in

Additional_file_A8.pdf). This contrasts the situation in many bacteria, such as Yersinia and Portiera (Parkhill et al. 2001; Sloan and Moran 2013), in which there have been substantial changes in gene arrangement among closely related strains. The source of this variation has been ascribed not only to the differences among species in their repertoires of DNA recombination and repair enzymes (Tamas et al. 2002) but also to selection on gene order and position (Suyama and Bork 2001; Ballouz et al. 2010; Treangen and Rocha 2011). Our analyses show that some fraction of the rearrangements that shuffle IGRs may affect organismal fitness by disrupting or generating regulatory elements. Although the IGR between yag $U$ and $\operatorname{ompN}$ was disrupted and split in the Salmonella genome, some portions of it-approximately $70 \mathrm{nt}$ of the 5'-end of EcsR1 and 20 nt of its 3 '-end - are still recognizable adjacent to the $u s p F$ and $o m p N$ genes in Salmonella (Figure B.5). It is likely that these sRNA segments are not transcribed and are not functional in Salmonella (supplementary fig. S4, found in Additional_file_A8.pdf, and Kroger et al. 2013) because nucleotide substitutions in the putative CRP- and FNR-binding regions (supplementary fig. S6, found in Additional_file_A8.pdf) have rendered them inactive. The reintroduction of EcsR1 into Salmonella did not affect biofilm production but instead triggered the increased 
expression of several virulence genes, particularly those within the SPI-1 pathogenicity island (supplementary table S7, found in Additional_file_A8.pdf). Multiple factors, including the biofilm machinery, are known to regulate the expression of these invasionassociated genes (Fàbrega and Vila 2013), suggestive of links between the different phenotypes produced by this sRNA in E. coli and Salmonella. Additional experiments are necessary to understand how EcsR1 induces diverse phenotypes in the two species; nevertheless, our findings demonstrate the potential of sRNAs to influence bacterial adaptation and evolution.

In addition to losing the biofilm-reducing sRNA (EcsR1), Salmonella has gained, again by a rearrangement event, an IGR that contains an sRNA (SesR2) that is not present in other enteric species. Because none of the corresponding regions in E. coli displays any appreciable transcript production, the evolution of this new sRNA in Salmonella also required the de novo formation of a new promoter sequence (Figure B.3 and supplementary fig. S3, found in Additional_file_A8.pdf). In bacterial genomes, $\sigma^{70}$ promoter-like sequences are able to arise spontaneously through point mutations, especially in IGRs (Stone and Wray 2001; Huerta et al. 2006; Mendoza-Vargas et al. 2009), and transcription can originate from newly evolved $\sigma^{70}$ promoters (MendozaVargas et al. 2009; Raghavan et al. 2012). Therefore, it is most likely that an incipient promoter in the newly formed STM14_1869-STM14_1870 IGR gave rise to the transcript that evolved into SesR2. An alternate possibility is that SesR2 was introduced into Salmonella with the HGT event that brought in the entire STM14_1870STM14_1872 region, as has been proposed for other sRNAs located close to transposon 
insertion sites in Salmonella (Sittka et al. 2008). However, no similar sRNA is detectable in the homologous regions found on various enteric plasmids and genomes, making this scenario less likely. Finally, because the first $55 \mathrm{nt}$ of this sRNA is complementary to the 5'-UTR of STM14_1869 (TSS of STM14_1869 is located 63 bp upstream of coding region), it could be functioning as an antisense RNA to regulate STM14_1869 expression, as shown previously for other genes in Salmonella (Lee and Groisman 2010). In bacteria, differences in protein-coding gene contents between closely related species are either due to new genes that arose by gene duplication or HGT (Lerat et al. 2005; Blount et al. 2012; Nasvall et al. 2012), or due to gene loss through pseudogenization and deletion (Mira et al. 2001; Kuo and Ochman 2010). Although the mechanisms that shape bacterial sRNA gene repertoires are not well understood, duplication, deletion, and HGT have also been attributed to this process (Gottesman and Storz 2011). In this report, we show that genome rearrangements that create and disrupt IGRs can result in the gain or loss of sRNA genes in bacteria. Because sRNAs regulate myriad metabolic processes, this disparity in sRNA repertoires between closely related bacteria might also contribute to niche adaptation and speciation events. 


\section{B.6 REFERENCES}

Anantharaman V, Aravind L. New connections in the prokaryotic toxin-antitoxin network: relationship with the eukaryotic nonsense-mediated RNA decay system. Genome Biol. 2003;4:R81.

Anders S, Huber W. Differential expression analysis for sequence count data. Genome Biol. 2010;11:R106.

Baba T, et al. Construction of Escherichia coli K-12 in-frame, single-gene knockout mutants: the Keio collection. Mol Syst Biol. 2006;2:1216-1226.

Ballouz S, Francis AR, Lan R, Tanaka MM. Conditions for the evolution of gene clusters in bacterial genomes. PLoS Comput Biol. 2010;6:e1000672.

Beisel CL, Storz G. The base-pairing RNA spot 42 participates in a multioutput feedforward loop to help enact catabolite repression in Escherichia coli. Mol Cell. 2011;41:286-297.

Blount ZD, Barrick JE, Davidson CJ, Lenski RE. Genomic analysis of a key innovation in an experimental Escherichia coli population. Nature. 2012;489:513-518.

Bobay LM, Rocha EP, Touchon M. The adaptation of temperate bacteriophages to their host genomes. Mol Biol Evol. 2013;30:737-751.

Brüssow H, Canchaya C, Hardt WD. Phages and the evolution of bacterial pathogens: from genomic rearrangements to lysogenic conversion. Microbiol Mol Biol Rev. 2004;68:560-602. 
Chao Y, Papenfort K, Reinhardt R, Sharma CM, Vogel J. An atlas of Hfq-bound transcripts reveals 3' UTRs as a genomic reservoir of regulatory small RNAs. EMBO J. 2012;31:4005-4019.

Constantinidou $\mathrm{C}$, et al. A reassessment of the FNR regulon and transcriptomic analysis of the effects of nitrate, nitrite, NarXL, and NarQP as Escherichia coli K12 adapts from aerobic to anaerobic growth. J Biol Chem. 2006;281:4802-4815.

Datsenko KA, Wanner B. One-step inactivation of chromosomal genes in Escherichia coli K-12 using PCR products. Proc Natl Acad Sci U S A. 2000;97:6640-6645.

De Lay N, Schu DJ, Gottesman S. Bacterial small RNA-based negative regulation: Hfq and its accomplices. J Biol Chem. 2013;288:7996-8003.

Dornenburg JE, Devita AM, Palumbo MJ, Wade JT. Widespread antisense transcription in Escherichia coli. mBio. 2010;1:e0024-10.

Durand S, Storz G. Reprogramming of anaerobic metabolism by the FnrS small RNA. Mol Microbiol. 2010;75:1215-1231.

Fàbrega A, Vila J. Salmonella enterica serovar Typhimurium skills to succeed in the host: virulence and regulation. Clin Microbiol Rev. 2013;26:308-341.

Gottesman S, Storz G. Bacterial small RNA regulators: versatile roles and rapidly evolving variations. Cold Spring Harb Perspect Biol. 2011;3:a003798.

Gualdi L, et al. Cellulose modulates biofilm formation by counteracting curli-mediated colonization of solid surfaces in Escherichia coli. Microbiology. 2008;154:20172024. 
Guo MS, et al. MicL, a new $\sigma E$-dependent sRNA, combats envelope stress by repressing synthesis of Lpp, the major outer membrane lipoprotein. Genes Dev. 2014;28: $1620-1634$.

Horler RS, Vanderpool CK. Homologs of the small RNA SgrS are broadly distributed in enteric bacteria but have diverged in size and sequence. Nucleic Acids Res. 2009; $37: 5465-5476$.

Huang DW, Sherman BT, Lempicki RA. Systematic and integrative analysis of large gene lists using DAVID Bioinformatics Resources. Nat Protoc. 2009;4:44-57.

Huerta AM, Collado-Vides J. Sigma70 promoters in Escherichia coli: specific transcription in dense regions of overlapping promoter-like signals. J Mol Biol. 2003;333:261-278.

Huerta AM, Francino MP, Morett E, Collado-Vides J. Selection for unequal densities of sigma70 promoter-like signals in different regions of large bacterial genomes. PLoS Genet. 2006;2:e185.

Kaessmann H. Origins, evolution, and phenotypic impact of new genes. Genome Res. 2010;20:1313-1326.

Karatan E, Watnick P. Signals, regulatory networks, and materials that build and break bacterial biofilms. Microbiol Mol Biol Rev. 2009;73:310-347.

Keseler IM, et al. EcoCyc: fusing model organism databases with systems biology. Nucleic Acids Res. 2013;41:D605-D612.

Kroger C, et al. The transcriptional landscape and small RNAs of Salmonella enterica serovar Typhimurium. Proc Natl Acad Sci U S A. 2012;109:E1277-E1286. 
Kroger C, et al. An infection-relevant transcriptomic compendium for Salmonella enterica serovar Typhimurium. Cell Host Microbe. 2013;14:683-695.

Kuo CH, Ochman H. The extinction dynamics of bacterial pseudogenes. PLoS Genet. 2010;6:e1001050.

Lee EJ, Groisman EA. An antisense RNA that governs the expression kinetics of a multifunctional virulence gene. Mol Microbiol. 2010;76:1020-1033.

Lenz DH, et al. The small RNA chaperone Hfq and multiple small RNAs control quorum sensing in Vibrio harveyi and Vibrio cholerae. Cell. 2004;118:69-82.

Lerat E, Daubin V, Ochman H, Moran NA. Evolutionary origins of genomic repertoires in bacteria. PLoS Biol. 2005;3:e130.

Li H, Ruan J, Durbin R. Mapping short DNA sequencing reads and calling variants using mapping quality scores. Genome Res. 2008;18:1851-1858.

Long M, Langley $\mathrm{CH}$. Natural selection and the origin of jingwei, a chimeric processed functional gene in Drosophila. Science. 1993;260:91-95.

Mahadevan P, Seto D. Rapid pair-wise synteny analysis of large bacterial genomes using web-based GeneOrder4.0. BMC Res Notes. 2010;3:41.

Mendoza-Vargas A, et al. Genome-wide identification of transcription start sites, promoters and transcription factor binding sites in E. coli. PLoS One. 2009; 4:e7526.

Mira A, Ochman H, Moran NA. Deletional bias and the evolution of bacterial genomes. Trends Genet. 2001;17:589-596. 
Nasvall J, Sun L, Roth JR, Andersson DI. Real-time evolution of new genes by innovation, amplification, and divergence. Science. 2012;338:384-387.

Ochman H, Elwyn S, Moran NA. Calibrating bacterial evolution. Proc Natl Acad Sci. 1999;96:12638-12643.

Parkhill J, et al. Genome sequence of Yersinia pestis, the causative agent of plague. Nature. 2001;413:523-527.

Peer A, Margalit H. Evolutionary patterns of Escherichia coli small RNAs and their regulatory interactions. RNA. 2014;20:994-1003.

Petty NK, et al. The Citrobacter rodentium genome sequence reveals convergent evolution with human pathogenic Escherichia coli. J Bacteriol. 2010;192:525538.

Pichon C, Felden B. Small RNA genes expressed from Staphylococcus aureus genomic and pathogenicity islands with specific expression among pathogenic strains. Proc Natl Acad Sci U S A. 2005;102:14249-14254.

Raghavan R, Groisman EA, Ochman H. Genome-wide detection of novel regulatory RNAs in E coli. Genome Res. 2011;21:1487-1497.

Raghavan R, Sage A, Ochman H. Genome-wide identification of transcription start sites yields a novel thermosensing RNA and new cyclic AMP receptor proteinregulated genes in Escherichia coli. J Bacteriol. 2011;193:2871-2874.

Raghavan R, Sloan DB, Ochman H. Antisense transcription is pervasive but rarely conserved in enteric bacteria. mBio. 2012;3:e00156-12. 
Rocha EP. Order and disorder in bacterial genomes. Curr Opin Microbiol. 2004;7:519527.

Rutherford K, et al. Artemis: sequence visualization and annotation. Bioinformatics. 2000;16:944-945.

Sittka A, et al. Deep sequencing analysis of small noncoding RNA and mRNA targets of the global post-transcriptional regulator, Hfq. PLoS Genet. 2008;4:e1000163.

Skippington E, Ragan MA. Evolutionary dynamics of small RNAs in 27 Escherichia coli and Shigella genomes. Genome Biol Evol. 2012;4:330-345.

Sloan DB, Moran NA. The evolution of genomic instability in the obligate endosymbionts of whiteflies. Genome Biol Evol. 2013;5:783-793.

Stone JR, Wray GA. Rapid evolution of cis-regulatory sequences via local point mutations. Mol Biol Evol. 2001;18:1764-1770.

Storz G, Vogel J, Wassarman KM. Regulation by small RNAs in bacteria: expanding frontiers. Mol Cell. 2011;43:880-891.

Suyama M, Bork P. Evolution of prokaryotic gene order: genome rearrangements in closely related species. Trends Genet. 2001;17:10-13.

Tamas I, et al. 50 million years of genomic stasis in endosymbiotic bacteria. Science. 2002;296:2376-2379.

Tjaden B, et al. Transcriptome analysis of Escherichia coli using high-density oligonucleotide probe arrays. Nucleic Acids Res. 2002;30:3732-3738.

Treangen TJ, Rocha EP. Horizontal transfer, not duplication, drives the expansion of protein families in prokaryotes. PLoS Genet. 2011;7:e1001284. 
Unterholzner SJ, Hailer B, Poppenberger B, Rozhon W. Characterisation of the stbD/E toxin-antitoxin system of pEP36, a plasmid of the plant pathogen Erwinia pyrifoliae. Plasmid. 2013;70:216-225.

Van Alst NE, Picardo KF, Iglewski BH, Haidaris CG. Nitrate sensing and metabolism modulate motility, biofilm formation, and virulence in Pseudomonas aeruginosa. Infect Immun. 2007;75:3780-3790.

Wassarman KM, Storz G. 6S RNA regulates E. coli RNA polymerase activity. Cell. 2000;101:613-623.

Wilderman PJ, et al. Identification of tandem duplicate regulatory small RNAs in Pseudomonas aeruginosa involved in iron homeostasis. Proc Natl Acad Sci. 2004;101:9792-9797. 


\begin{abstract}
APPENDIX C
Emergence of new sRNAs in enteric bacteria is associated with low expression and rapid evolution
\end{abstract}

\title{
C.1 ABSTRACT
}

Non-coding small RNAs (sRNAs) are critical to post-transcriptional gene regulation in bacteria. However, unlike for protein-coding genes, the evolutionary forces that shape sRNAs are not understood. We investigated sRNAs in enteric bacteria and discovered that recently emerged sRNAs evolve at significantly faster rates than older sRNAs. Concomitantly, younger sRNAs are expressed at significantly lower levels than older sRNAs. This process could potentially facilitate the integration of newly emerged sRNAs into bacterial regulatory networks. Furthermore, it has previously been difficult to trace the evolutionary histories of sRNAs because rapid evolution obscures their original sources. We overcame this challenge by identifying a recently evolved sRNA in Escherichia coli, which allowed us to determine that novel sRNAs could emerge from vestigial bacteriophage genes, the first known source for sRNA origination. 


\section{C.2 INTRODUCTION}

Non-coding RNAs (ncRNAs) regulate gene expression in all domains of life. In Eukaryotes, post-transcriptional control of gene expression by ncRNA such as microRNA (miRNA) and small interfering RNA is now recognized as a fundamental layer of gene regulation (Wilson and Doudna 2013). While less studied, Archaea also contain a large repertoire of ncRNAs (Bernick et al. 2012). In Bacteria, a major class of ncRNA is small RNAs (sRNAs), which are around 50-200 nucleotides in length, and regulate gene expression by binding to messenger RNAs (mRNAs) (Gottesman and Storz 2011). Broadly, sRNAs are classified into two major types: (1) trans-acting sRNAs that are encoded in intergenic regions and regulate the expression of distantly located genes via imperfect complementarity, and (2) cis-acting sRNAs (also called antisense RNAs) that are transcribed from the opposite strand of adjacent target genes and regulate gene expression through perfectly complementary regions (Thomason and Storz 2010; Georg and Hess 2011). Some of the other major classes of bacterial ncRNA are riboswitches and RNA thermometers that are located in the untranslated regions of certain mRNAs (Breaker 2011; Kortmann and Narberhaus 2012), and intraRNAs that originate from within protein-coding genes (Miyakoshi et al. 2015).

Several advantages of sRNAs over proteins have led to their emergence as important gene regulators in bacteria. For instance, relatively lower energy is required to synthesize sRNAs; they act rapidly and their co-degradation along with target mRNAs allows precise control of regulatory circuits (Storz et al. 2011; Updegrove et al. 2015). 
Each sRNA typically controls multiple genes belonging to interconnected processes, including metabolic pathways, quorum sensing, biofilm formation, and virulence (Michaux et al. 2014), and because there are hundreds of sRNAs in each bacterium, their contribution to bacterial adaptation and phenotypic diversity could be tantamount to that of protein-coding genes (Gottesman and Storz 2011; Raghavan et al. 2011; Kröger et al. 2013). Hence, differences in sRNA sequences and contents between closely related bacteria could have a substantial impact on bacterial physiology and pathogenicity. However, we have minimal knowledge about the evolutionary forces that shape bacterial sRNAs. An analysis of the conservation of sRNAs in Escherichia coli showed that variation in sRNA contents between strains is mainly due to deletions (Skippington and Ragan 2012), and a broader examination of sRNA conservation across bacteria revealed that most $E$. coli sRNAs originated after enteric bacteria split from other Gammaproteobacteria (Peer and Margalit 2014). In addition to this cycle of birth and loss, sRNA genes evolve at faster rates than protein-coding genes, making it difficult to identify sRNA homologs in distantly related bacteria (Gardner et al. 2005; Hoeppner et al. 2012). One group of bacteria that was shown to be at optimum evolutionary distance for effective evolutionary analysis of sRNAs is the family Enterobacteriaceae (Lindgreen et al. 2014), which includes E. coli and Salmonella enterica, two model organisms in which sRNAs have been investigated thoroughly (e.g., Raghavan et al. 2011; Kröger et al. 2013).

In this study, we estimated the evolutionary ages of $>200$ sRNAs present in $E$. coli and S. enterica, and show that younger sRNAs are expressed at significantly lower 
levels than older sRNAs, and that younger sRNAs have significantly higher rates of evolution than older sRNAs. The low expression of new sRNAs could mitigate the negative effects of nascent sRNA-mRNA interactions, whereas their rapid evolution could generate beneficial interactions that facilitate their integration into bacterial regulatory networks. We also show that most sRNAs are evolving under purifying selection, and discovered that a young sRNA in E. coli originated from a vestigial bacteriophage protein-coding gene, thereby revealing the first known source for sRNA origination in bacteria. 


\section{C.3 MATERIALS AND METHODS}

\section{Bacterial Strains and Plasmids}

Escherichia coli K-12 MG1655 was used in all experiments. For EcsR2 expression vector construction, EcsR2 gene was amplified using the following primers: 5'ATGCTAGCGCAGATAGTCAGTGAGTATATC3', 5'GACGTCGCAGATAGTCAGTGAGTATATC3', and cloned into the plasmid pBAD (Guzman et al. 1995) by digesting both the PCR product and $\mathrm{pBAD}$ with NheI and AatII restriction enzymes (restriction sites on primers are underlined). EcsR2-deletion strain was constructed using $\lambda$ Red-mediated recombination (Datsenko and Wanner 2000).

\section{RNA-Seq and Crosslink-Seq}

Highest level of EcsR2 expression was observed during exponential phase growth (Fig. S4, found in Additional_file_A9.pdf). Hence, for the RNA-seq analysis, $E$. coli transformed with either empty pBAD (control) or pBAD with cloned EcsR2 (test) that were grown in Lysogeny Broth (LB) aerobically to $\mathrm{OD}_{600}$ of $\sim 0.5$. Cultures were supplemented with arabinose $(0.2 \%)$ for $10 \mathrm{~min}$ to induce the expression of EcsR2, 0.2 volume stop solution ( $5 \%$ water-saturated phenol, $95 \%$ ethanol) was added, and the cells were harvested by centrifugation. Total RNA was extracted using TRI reagent, treated with DNase, and ribosomal RNAs were removed using MICROBExpress kit (Life Technologies). RNA-seq (Illumina HiSeq 2000, 100 cycles, single-end) was performed using two control and test samples at the Genomic Sequencing and Analysis Facility at 
the University of Texas at Austin. The trimmed reads were mapped to the E. coli genome (NC_000913.2) using CLC Genomics Workbench to identify genes that were differentially expressed. The RNA-seq reads are available on NCBI SRA (accession: SRP044074).

Crosslink-seq was adapted from Lustig et al. 2010 and Liu et al. 2015. EcsR2deletion strain containing either empty pBAD (control) or pBAD with cloned EcsR2 (test) were grown in LB aerobically to $\mathrm{OD}_{600}$ of $\sim 0.5$ and cultures were supplemented with arabinose $(0.2 \%)$ for 10 min to induce the expression of EcsR2. Cells were washed twice with PBS, resuspended in $8 \mathrm{~mL}$ of fresh PBS, and $0.2 \mathrm{mg} / \mathrm{mL} \mathrm{4'-}$ Aminomethyltrioxsalen (Cayman Chemicals) was added. The cells were incubated on ice for $10 \mathrm{~min}$, and were irradiated with UV light at $365 \mathrm{~nm}$ for $1 \mathrm{~h}$ on ice. The cells were washed once with PBS and total RNA was isolated using TRI reagent. RNA treated with DNase was mixed in hybridization buffer (20 nM HEPES pH8, $5 \mathrm{mM} \mathrm{MgCl} 2,300 \mathrm{mM}$ $\mathrm{KCl}, 0.01 \% \mathrm{NP}-40,1 \mathrm{mM} \mathrm{DTT})$ and heated at $80{ }^{\circ} \mathrm{C}$ for $2 \mathrm{~min}$ followed by immediate cooling on ice. $10 \mathrm{nmol}$ biotinylated oligonucleotides that were antisense to a portion of EcsR2 were added to the samples and incubated at room temperature overnight. $150 \mu \mathrm{L}$ of NeutrAvidin agarose resin (Thermo Fisher) was washed twice in WB100 buffer (20 mM HEPES pH 8, $10 \mathrm{mM} \mathrm{MgCl} 2,100 \mathrm{mM} \mathrm{KCl,} \mathrm{0.01 \%} \mathrm{NP-40,} 1 \mathrm{mM}$ DTT) followed by blocking the beads for $2 \mathrm{~h}$ (blocking buffer: WB100, $50 \mu \mathrm{L}$ BSA $(10 \mathrm{mg} / \mathrm{mL}), 40 \mu \mathrm{L}$ tRNA $(10 \mathrm{mg} / \mathrm{mL}), 10 \mu \mathrm{L}$ glycogen $(20 \mathrm{mg} / \mathrm{mL}))$. The blocked beads were once again washed with blocking buffer and added to the hybridized RNAs bound to the biotinylated oligos. Samples were incubated for $4 \mathrm{~h}$ at $4{ }^{\circ} \mathrm{C}$ and then washed five times with WB400 
buffer (20 mM HEPES pH 8, 10 mM MgCl2, $400 \mathrm{mM} \mathrm{KCl,} \mathrm{0.01 \%} \mathrm{NP40,} 1$ mM DTT). The hybridized RNAs bound to the beads were isolated using TRI reagent. The affinityselected, crosslinked mRNAs were released from EcsR2 using UV light at $254 \mathrm{~nm}$ on ice for $15 \mathrm{~min}$. The RNA samples were deep-sequenced at Oregon Health and Science University Massively Parallel Sequencing Shared Resource (Illumina HiSeq 2500, 100 cycles, single-end), and the trimmed reads were mapped to E. coli genome (NC_000913.2) using CLC Genomics Workbench to determine the genes that were enriched in test samples (expressing EcsR2) in comparison to controls (no EcsR2). Gene expression was calculated from two independent experiments, and the RNA-seq reads are available on NCBI SRA (accession: SRP074317).

For qRT-PCR confirmation, EcsR2-deletion strain containing empty pBAD, or pBAD with cloned full-length EcsR2, or pBAD with EcsR2 in which the +51 to +80 region was deleted using inverse PCR were used. Bacteria were grown in LB aerobically to $\mathrm{OD}_{600}$ of $\sim 0.5$. Cultures were supplemented with arabinose $(0.2 \%)$ for $10 \mathrm{~min}$ to induce the expression of EcsR2, and 0.2 volume stop solution was immediately added, and the cells were harvested by centrifugation. Total RNA was extracted using TRI reagent, treated with DNase, and qRT-PCR was performed as previously described (Raghavan et al. 2011).

\section{Expression and Evolution of sRNAs}

We used previously published RNA-seq data to determine the expression of sRNAs (Raghavan et al. 2011; Kröger et al. 2013). To identify the homologs of 92 sRNAs 
described in E. coli K-12 MG1655 (NC_000913.2) (Raghavan et al. 2011, 2015), we used BLASTn ( $E$ value $<10^{-5}$ and target length $\geq 60 \%$ of query length) to search 146 fully sequenced $E$. coli genomes available on NCBI. We ultimately chose 81 sRNAs that were conserved in $85 \mathrm{E}$. coli genomes in order to maximize the number of genomes and sRNAs (Supplemental dataset 1). Similarly, we searched 151 S. enterica genomes to identify the homologs of 170 sRNAs described in S. enterica Typhimurium SL1344 (NC_016810.1) (Kröger et al. 2013), and chose 127 sRNAs that are conserved in 112 S. enterica genomes for further analyses (Supplemental dataset 1). Sequences were aligned using Clustal Omega (Sievers et al. 2011), and nucleotide differences were quantified using nucleotide diversity index $\pi$ (Nei 1987; Jovelin and Cutter 2014) with DnaSP 5.10 (Librado and Rozas 2009). Briefly, $\pi$ was calculated by summing, over all distinct pairs of sequences in the sample, the proportion of different nucleotides between a pair of sequences multiplied by the respective frequencies of those sequences. To calculate the average nucleotide differences throughout the $y a g U-y k j G$ IGR, we used a sliding window of $35 \mathrm{bp}$ and a step size of $15 \mathrm{bp}$ using DnaSP. RNA secondary structure and minimum free energy were predicted using Vienna RNA webserver (Gruber et al. 2008) and Mfold webserver (Zuker 2003), and EcsR2-AnsB interaction was modeled using IntaRNA (Wright et al. 2014).

To determine whether the sRNAs in E. coli and S. enterica were present in other enteric bacteria, sRNA gene sequences were searched (BLASTn, $E<10^{-5}$ and target length $\geq 60 \%$ of query length) against the following representative genomes (as denoted by NCBI Genome database): Citrobacter freundii (NZ_CP007557.1), Klebsiella 
pneumoniae (NC_016845.1), Serratia marcescens (NZ_HG326223.1), and Yersinia enterocolitica (NC_008800.1). PMCMR R package was used to perform both KruskalWallis test (non-parametric 1-way ANOVA) to assess differences in expression and nucleotide diversity between sRNA age classes, and the post hoc pairwise comparison Dunn's test. For analyzing S. enterica expression data from 22 growth conditions (Kröger et al. 2013), Permutational ANOVA (non-parametric 2-way ANOVA) was conducted using perm.anova, and post hoc pairwise comparisons were conducted using pairwise.perm.t.test with FDR correction in the RVAideMemoire R package. To analyze sRNA evolution in more detail, 38 sRNAs that were conserved in at least $50 \%$ of currently available complete genomes of E. coli (74 strains) and S. enterica (102 strains) were chosen (Table C.1). To detect purifying selection, within-species polymorphism and between-species divergence were calculated using DnaSP; four-fold degenerate sites (in hundred randomly selected genes; Table C.2) were used as control because they are considered to evolve neutrally (Ochman and Wilson 1987). 
Table C.1 - Genomes of $E$. coli (85 strains) and $S$. enterica (102 strains) used in analysis

\begin{tabular}{|c|c|}
\hline Sample & Genes \\
\hline $\begin{array}{l}\text { E. coli } \\
\text { genomes }\end{array}$ & $\begin{array}{l}\text { NC_000913.2, NC_004431.1, NC_007779.1, NC_007946.1, NC_008563.1, } \\
\text { NC_009801.1, NC_010468.1, NC_010498.1, NC_011415.1, NC_011741.1, } \\
\text { NC_011748.1, NC_011751.1, NC_011993.1, NC_013353.1, NC_013941.1, } \\
\text { NC_016902.1, NC_017625.1, NC_017626.1, NC_017628.1, NC_017631.1, } \\
\text { NC_017632.1, NC_017633.1, NC_017634.1, NC_017635.1, NC_017638.1, } \\
\text { NC_017641.1, NC_017651.1, NC_017652.1, NC_017656.1, NC_017664.1, } \\
\text { NC_018650.1, NC_018658.1, NC_018661.1, NC_020163.1, NC_022364.1, } \\
\text { NC_022370.1, NZ_CP005930.1, NZ_CP006027.1, NZ_CP006262.1, NZ_CP006632.1, } \\
\text { NZ_CP007133.1, NZ_CP007136.1, NZ_CP007149.1, NZ_CP007265.1, } \\
\text { NZ_CP007390.1, NZ_CP007391.1, NZ_CP007392.1, NZ_CP007393.1, } \\
\text { NZ_CP007442.1, NZ_CP007491.1, NZ_CP007592.1, NZ_CP007594.1, } \\
\text { NZ_CP008801.1, NZ_CP009072.1, NZ_CP009104.1, NZ_CP009106.2, } \\
\text { NZ_CP009166.1, NZ_CP009273.1, NZ_CP009685.1, NZ_CP010344.1, } \\
\text { NZ_CP010445.1, NZ_CP011018.1, NZ_CP011113.1, NZ_CP011134.1, } \\
\text { NZ_CP011320.1, NZ_CP011321.1, NZ_CP011324.1, NZ_CP011331.1, } \\
\text { NZ_CP011342.2, NZ_CP011343.2, NZ_CP011416.1, NZ_CP011495.1, } \\
\text { NZ_CP012125.1, NZ_CP012126.1, NZ_CP012127.1, NZ_CP012625.1, } \\
\text { NZ_CP012631.1, NZ_CP012635.1, NZ_CP012868.1, NZ_CP012869.1, } \\
\text { NZ_CP012870.1, NZ_CP013025.1, NZ_CP013112.1, NZ_CP014225.1, } \\
\text { NZ_HF572917.1 }\end{array}$ \\
\hline $\begin{array}{l}\text { S. enterica } \\
\text { genomes }\end{array}$ & $\begin{array}{l}\text { NC_003197.1, NC_006905.1, NC_010102.1, NC_011080.1, NC_011083.1, } \\
\text { NC_011205.1, NC_011274.1, NC_011294.1, NC_012125.1, NC_016810.1, } \\
\text { NC_016831.1, NC_016854.1, NC_016856.1, NC_016857.1, NC_016863.1, } \\
\text { NC_017046.1, NC_017623.1, NC_021151.1, NC_021810.1, NC_021812.2, } \\
\text { NC_021814.1, NC_021820.1, NC_021844.1, NC_021902.1, NC_022221.1, } \\
\text { NC_022525.1, NC_022544.1, NC_022569.1, NZ_AP014565.1, NZ_CP005995.1, } \\
\text { NZ_CP007175.1, NZ_CP007216.1, NZ_CP007235.1, NZ_CP007245.1, } \\
\text { NZ_CP007246.1, NZ_CP007247.1, NZ_CP007248.1, NZ_CP007249.2, } \\
\text { NZ_CP007250.1, NZ_CP007251.1, NZ_CP007252.1, NZ_CP007253.1, } \\
\text { NZ_CP007254.1, NZ_CP007258.1, NZ_CP007259.1, NZ_CP007260.1, } \\
\text { NZ_CP007261.1, NZ_CP007262.1, NZ_CP007263.1, NZ_CP007267.2, } \\
\text { NZ_CP007269.1, NZ_CP007271.1, NZ_CP007280.2, NZ_CP007282.2, } \\
\text { NZ_CP007283.2, NZ_CP007285.2, NZ_CP007286.2, NZ_CP007288.2, } \\
\text { NZ_CP007292.1, NZ_CP007294.2, NZ_CP007312.2, NZ_CP007319.1, } \\
\text { NZ_CP007333.1, NZ_CP007425.1, NZ_CP007426.1, NZ_CP007434.2, } \\
\text { NZ_CP007507.1, NZ_CP007523.1, NZ_CP007528.1, NZ_CP007534.1, } \\
\text { NZ_CP007559.1, NZ_CP007581.1, NZ_CP007598.1, NZ_CP008928.1, } \\
\text { NZ_CP009083.1, NZ_CP009084.2, NZ_CP009085.2, NZ_CP009086.1, } \\
\text { NZ_CP009087.1, NZ_CP009088.1, NZ_CP009089.1, NZ_CP009090.1, } \\
\text { NZ_CP009091.1, NZ_CP009092.1, NZ_CP009093.1, NZ_CP009102.1, } \\
\text { NZ_CP009561.1, NZ_CP009768.1, NZ_CP010279.1, NZ_CP010280.1, } \\
\text { NZ_CP010281.1, NZ_CP010282.1, NZ_CP010283.1, NZ_CP010284.1, } \\
\text { NZ_CP011394.1, NZ_CP011396.1, NZ_CP011428.1, NZ_CP011790.1, } \\
\text { NZ_CP011791.1, NZ_CP012144.1, NZ_CP012347.1, NZ_CP012513.1, } \\
\text { NZ_CP012514.1, NZ_CP012681.1, NZ_CP012921.1, NZ_CP012924.1, } \\
\text { NZ_CP012930.1, NZ_CP013097.1, NZ_CP014051.1, NZ_CP014356.1, } \\
\text { NZ_CP014358.1, NZ_CP014536.1 }\end{array}$ \\
\hline
\end{tabular}


Table C.2 - 100 genes selected in $E$. coli (NC_000913) and $S$. enterica $\left(\mathrm{NC} \_016810\right)$ selected for four-fold degenerate site analysis

\begin{tabular}{|c|c|}
\hline Sample & Genes \\
\hline E. coli genes & $\begin{array}{l}\text { aaeA, aaeB, aaeX, aas, aat, accA, accB, accC, accD, aceA, aceB, aceE, aceF, ackA, } \\
\text { acnA, acnB, acpH, acpP, acpS, acrA, acrB, acrD, acrF, acrR, acrZ, acs, actP, add, adiA, } \\
\text { adiC, adiY, adiY, adiY, adiY, adiY, adiY, adiY, adiY, adiY, adiY, alkA, alkB, allA, } \\
\text { allR, allS, alr, alx, amiA, amiB, amiD, amn, ampD, ampE, amyA, ansA, ansB, ansP, } \\
\text { apaG, apbE, aphA, apt, araC, araD, araJ, arcB, arfB, } \operatorname{argA}, \operatorname{argB}, \operatorname{argC}, \operatorname{argE}, \operatorname{argG}, \\
\text { argH, argO, argP, argR, argS, arnA, arnB, arnD, arnT, aroA, aroB, aroC, aroD, aroE, } \\
\text { aroF, aroG, aroH, aroK, aroL, aroM, aroP, artI, artJ, artM, artP, artQ, asd, asnA, asnB }\end{array}$ \\
\hline $\begin{array}{l}\text { S. enterica } \\
\text { genes }\end{array}$ & $\begin{array}{l}\text { SL1344_RS17380, SL1344_RS17375, SL1344_RS17385, SL1344_RS15575, } \\
\text { SL1344_RS04640, SL1344_RS01175, SL1344_RS17455, SL1344_RS17460, } \\
\text { SL1344_RS12140, SL1344_RS } 1480, \text { SL1344_RS21475, SL1344_RS00775, aceF, } \\
\text { SL1344_RS11990, SL1344_RS08555, SL1344_RS00805, SL1344_RS02065, } \\
\text { SL1344_RS05900, acpS, SL1344_RS02425, SL1344_RS02420, SL1344_RS12735, } \\
\text { SL1344_RS17515, SL1344_RS02430, SL1344_RS03950, SL1344_RS21940, } \\
\text { SL1344_RS21930, SL1344_RS07275, SL1344_RS22060, SL1344_RS22045, } \\
\text { SL1344_RS22050, SL1344_RS22050, SL1344_RS22050, SL1344_RS22050, } \\
\text { SL1344_RS22050, SL1344_RS22050, SL1344_RS22050, SL1344_RS22050, } \\
\text { SL1344_RS22050, SL1344_RS22050, SL1344_RS10920, SL1344_RS11630, } \\
\text { SL1344_RS02620, SL1344_RS02625, SL1344_RS02615, SL1344_RS21800, } \\
\text { SL1344_RS16665, SL1344_RS12580, SL1344_RS22375, SL1344_RS04520, } \\
\text { SL1344_RS10330, SL1344_RS00745, SL1344_RS00750, SL1344_RS09820, } \\
\text { SL1344_RS06430, SL1344_RS16065, SL1344_RS07895, SL1344_RS00455, } \\
\text { SL1344_RS11640, SL1344_RS21810, SL1344_RS02460, SL1344_RS00525, } \\
\text { SL1344_RS00510, SL1344_RS02015, SL1344_RS17190, SL1344_RS01215, } \\
\text { SL1344_RS15475, SL1344_RS21175, SL1344_RS21170, SL1344_RS21165, } \\
\text { SL1344_RS16990, SL1344_RS21180, SL1344_RS15865, SL1344_RS15850, } \\
\text { SL1344_RS17355, SL1344_RS09550, SL1344_RS11800, SL1344_RS11790, } \\
\text { SL1344_RS11805, SL1344_RS11810, SL1344_RS04755, SL1344_RS17990, } \\
\text { SL1344_RS12230, SL1344_RS06750, SL1344_RS17565, SL1344_RS13700, } \\
\text { SL1344_RS03850, SL1344_RS06695, SL1344_RS17995, SL1344_RS01985, } \\
\text { SL1344_RS01995, SL1344_RS00765, SL1344_RS04500, SL1344_RS04485, artM, } \\
\text { SL1344_RS04505, SL1344_RS04495, SL1344_RS18250, SL1344_RS19960, asnB }\end{array}$ \\
\hline
\end{tabular}




\section{C.4 RESULTS}

\section{Newly Evolved sRNAs have Low Expression and Rapid Rate of Evolution}

We identified the homologs of 81 E. coli sRNAs (Raghavan et al. 2011, 2015) and $127 S$. enterica Typhimurium sRNAs (Kröger et al. 2013) in Citrobacter freundii, Klebsiella pneumoniae, Serratia marcescens, and Yersinia enterocolitica using BLASTn. This approach has been shown to be effective at identifying sRNA homologs within Enterobacteriaceae (Skippington and Ragan 2012; Peer and Margalit 2014). We utilized maximum parsimony to estimate the age of each sRNA along a $16 \mathrm{~S}$ rDNA phylogenetic tree that encompasses the six enteric bacteria (Figure C.1), as done previously to study miRNA evolution (Lyu et al. 2014). We assigned the sRNAs into three age groups: old (those that originated in the common ancestor of all six bacteria), middle-aged (those that originated in the common ancestor of E. coli, S. enterica, C. freundii, and $K$. pneumoniae), and young (those that originated in the E. coli, S. enterica branch) (Figure C.1). Based on this classification, E. coli contained 21 young, 27 middle-aged, and 33 old sRNAs, whereas $S$. enterica contained 53 young, 48 middle-aged, and 26 old sRNAs (Additional_file_A10.csv, Additional_file_A11.csv).

We analyzed the expression of sRNAs using RNA-seq data for exponential phase growth of E. coli in Lysogeny Broth (Raghavan et al. 2011), and S. enterica Typhimurium in Lennox Broth (Kröger et al. 2013), and discovered that sRNA expression correlated with sRNA age: younger sRNAs have significantly lower expression than older sRNAs (Figure C.2, Additional_file_A10.csv, 


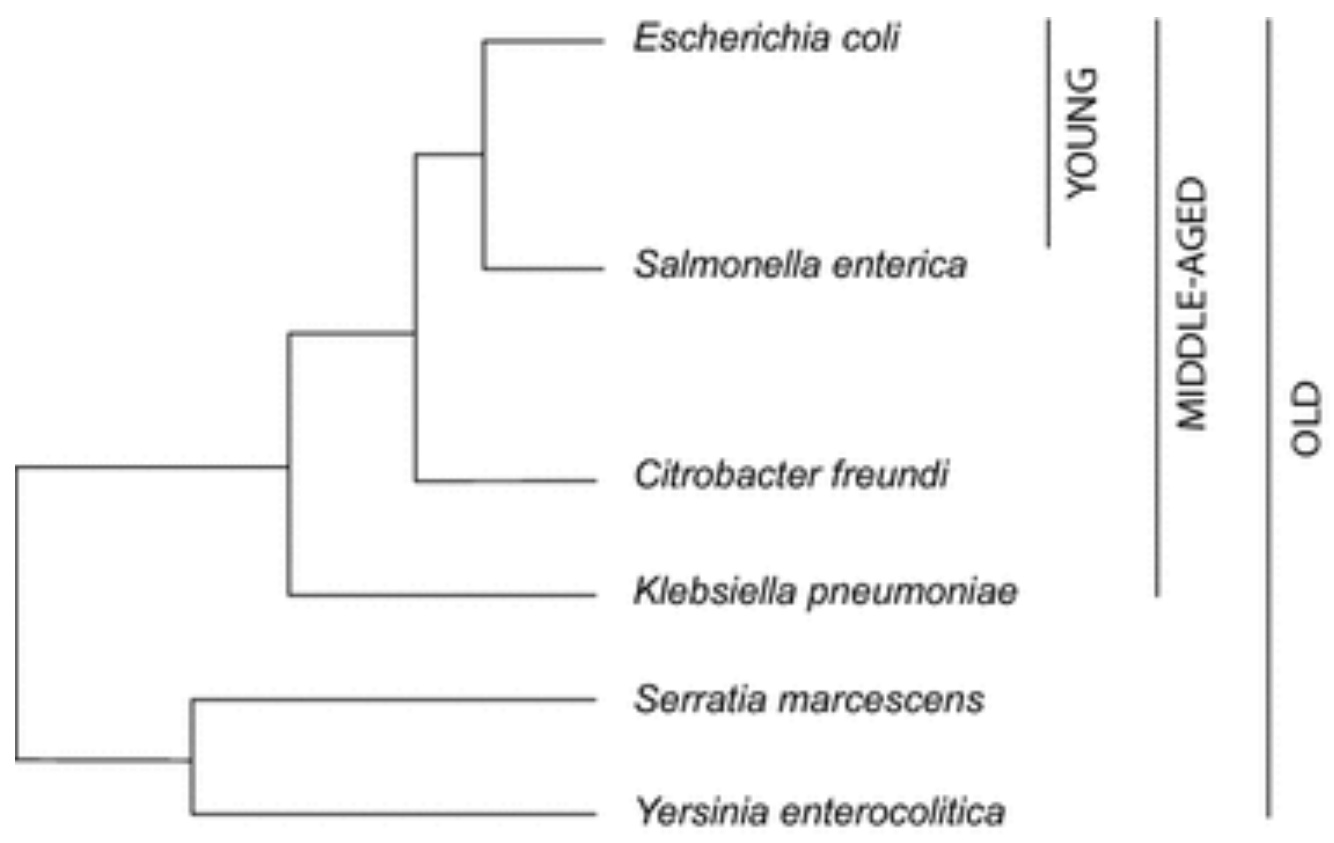

Figure C.1 - sRNA age groups. sRNAs were binned based on their presence in six enteric bacteria using maximum parsimony. 


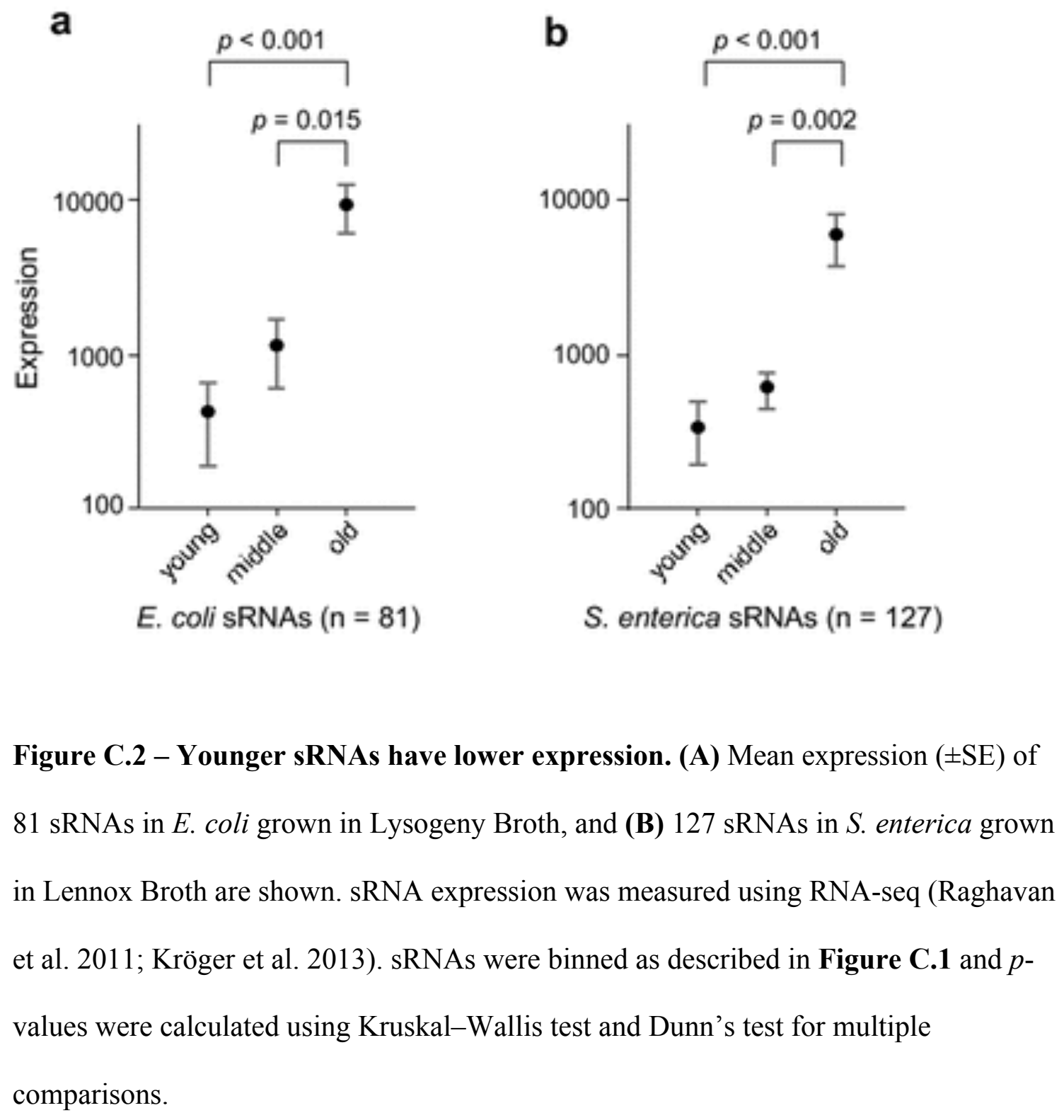


Additional_file_A11.csv). In order to rule out the possibility that the observed relationship between sRNA age and expression is an artifact of the growth conditions, we expanded our analysis of S. enterica sRNAs to all 22 "infection-relevant" growth conditions described by Kroger et al. (2013). Significantly reduced expression of young sRNAs in comparison to older sRNAs was observed under all growth conditions (Table S1, found in Additional_file_A9.pdf), showing that the relationship between sRNA age and expression is not dependent on the growth condition $(p=0.818$, Permutational ANOVA). Furthermore, to confirm that our conclusions are independent of the BLAST algorithm's ability to locate sRNA homologs in other enteric species, we performed a similar analysis using 49 E. coli sRNAs described in the Rfam database, which uses a different approach (covariance model) to identify homologous sRNAs (Nawrocki et al. 2015). As shown previously (Peer and Margalit 2014), we got comparable results using either BLASTn or Rfam (Table C.3, Additional_file_A12.csv).

We also measured the rate of evolution of each sRNA by calculating the nucleotide diversity index $\pi$, the average number of nt differences per site, using homologs in 85 E. coli and 112 S. enterica strains (Supplemental dataset 1) (Nei 1987; Jovelin and Cutter 2014). We discovered that the rate of sRNA evolution inversely correlated with age i.e., younger sRNAs evolved at significantly higher rates than older sRNAs (Figure C.3; Additional_file_A10.csv, Additional_file_A11.csv). Although we examined sRNAs only in enteric bacteria, the observed relationship among sRNA age, expression, and rate of evolution is likely to be a widespread phenomenon because previous studies in humans, nematodes, and Drosophila have shown that younger 
Table C.3 - Comparison of mean expression and nucleotide polymorphism of 49 rfam sRNAs in $\boldsymbol{E}$. coli grown in Lysogeny Broth. sRNA expression was measured using RNA-seq (Raghavan et al. 2011; Kröger et al. 2013). sRNAs were binned as described in Figure C.1 and $p$-values were calculated using Kruskal-Wallis test and Dunn's test for multiple comparisons.

\begin{tabular}{l|cc|cc} 
& \multicolumn{2}{c|}{ sRNA Expression } & \multicolumn{2}{c}{ Nucleotide Polymorphism } \\
Gene & Chi-Square & $\boldsymbol{p}$-value & Chi-Square & $\boldsymbol{p}$-value \\
\hline Overall & 13.13 & 0.001 & 10.69 & 0.005 \\
young vs. middle & 0.74 & 0.389 & 1.86 & 0.172 \\
middle vs. old & 7.84 & 0.005 & 4.36 & 0.037 \\
young vs. old & 9.48 & 0.002 & 9.33 & 0.002 \\
\hline
\end{tabular}




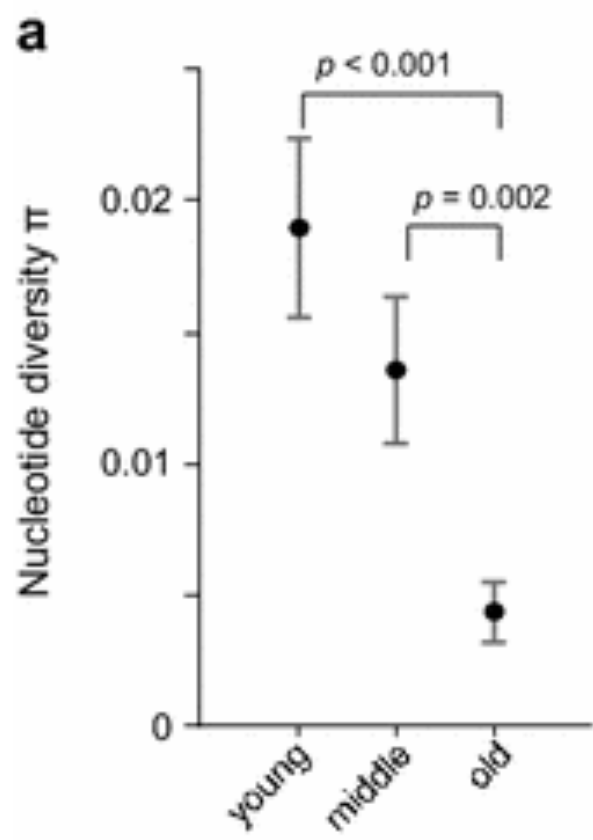

E. coli $\operatorname{sRNAs}(\mathrm{n}=81)$ b

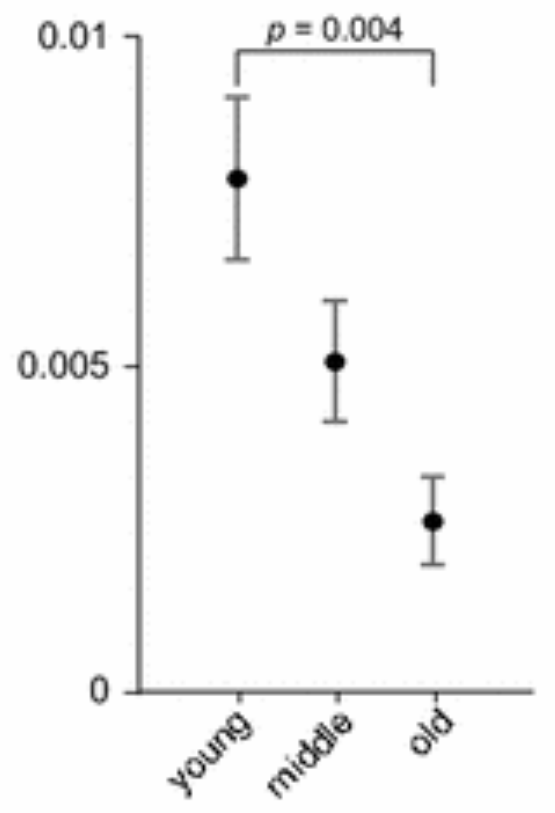

S. enterica sRNAs $(n=127)$

Figure C.3 - Younger sRNAs have higher rates of evolution. (A) Average ( \pm SE) $\pi$ values for 81 sRNAs in $E$. coli, and (B) 127 sRNAs in $S$. enterica are shown. sRNAs were binned as described in Figure C.1 and $p$-values were calculated using KruskalWallis test and Dunn's test for multiple comparisons. 
miRNAs have lower expression and faster rate of evolution than older miRNAs (Chen and Rajewsky 2007; Jovelin and Cutter 2014; Lyu et al. 2014).

\section{A Young sRNA in E. coli Originated from a Degraded Bacteriophage Gene}

One of the young sRNAs that had low expression and rapid rate of evolution was EcsR2 (Figure C.4), an sRNA found exclusively in E. coli (Raghavan et al. 2015). In order to understand the origin of EcsR2, we traced the evolutionary history of the $y a g U-y \mathrm{kgJ}$ intergenic region (IGR) that contains this sRNA. The arrangement in which yagU neighbors $y \mathrm{~kg} J$ is found only in $E$. coli, whereas an alternate, and likely ancestral, gene order $(y c i C-y k g J-o m p W)$ is present in most other enteric bacteria (Figure C.5). The ancestral gene arrangement is also present in E. albertii, which is one of E. coli's closest relatives, indicating that $y \mathrm{kgJ}$ moved to its current location in E. coli after the two bacteria split from a common ancestor. Additionally, the $y \mathrm{kgJ}$ ORF (open reading frame) is smaller in E. coli than in E. albertii, and $\sim 90$ bp remnant of the gene's $3^{\prime}$ end is still recognizable in the $y c i C$-ompW IGR in E. coli, confirming that $y \mathrm{~kg} J$ was translocated recently to its current location in $E$. coli to create the unique $y a g U-y \mathrm{~kg} J$ IGR (Figure C.5).

Due to their rapid rate of evolution, it is usually difficult to trace the ancestry of sRNAs (Gottesman and Storz 2011). However, because EcsR2 emerged in an IGR that was formed recently, we were able to identify through sequence alignment that the sRNA evolved from a vestigial bacteriophage gene (Figure C.6). To identify genes that are potentially regulated by EcsR2, we used RNA-seq to detect changes in mRNA levels in 
a

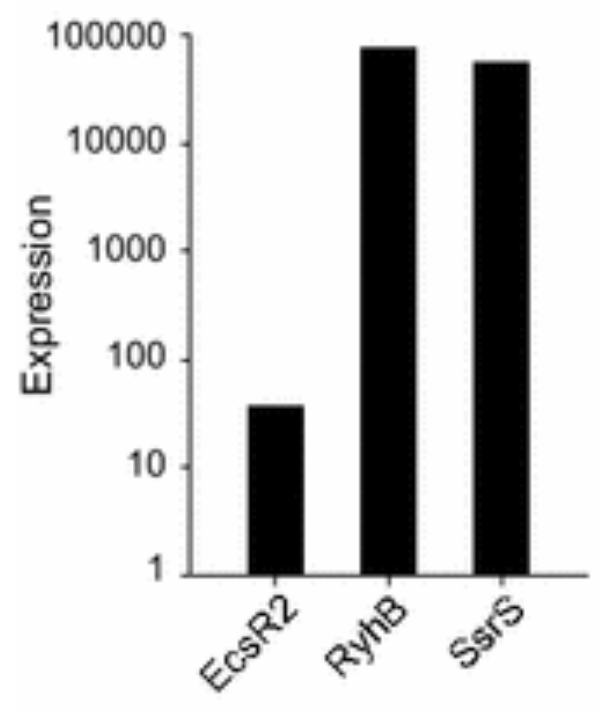

b

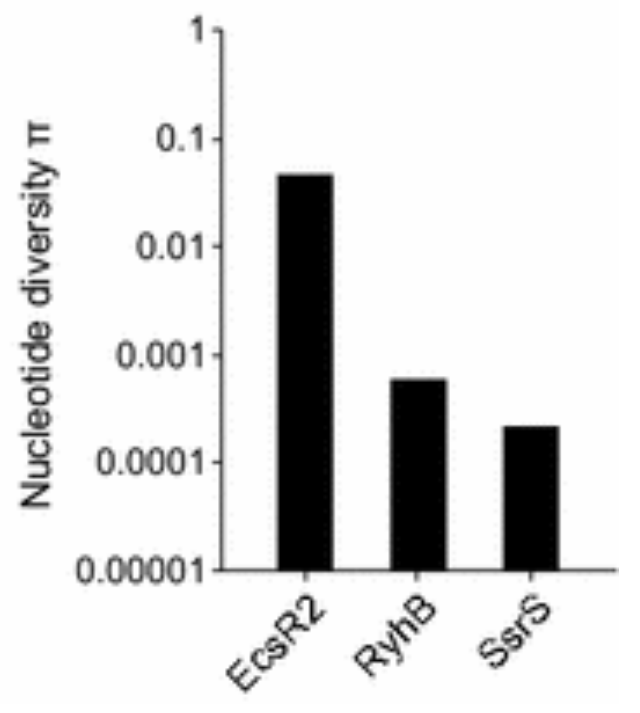

Figure C.4 - EcsR2 has low expression and rapid evolution. (A) Expression levels, and (B) nucleotide divergence index $(\pi)$ of EcsR2 compared to two old sRNAs (RyhB and $\mathrm{SsrS})$. 


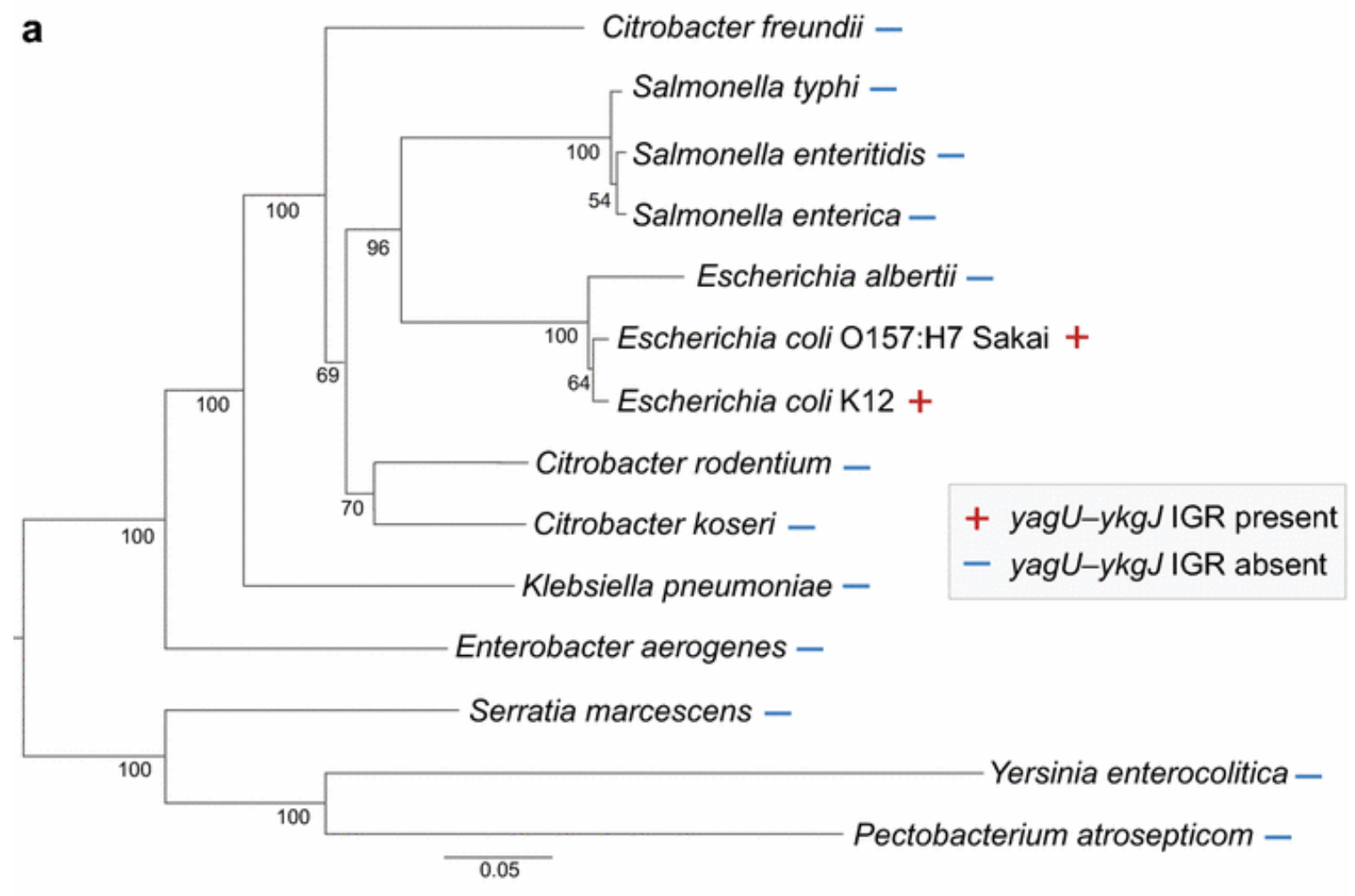

b

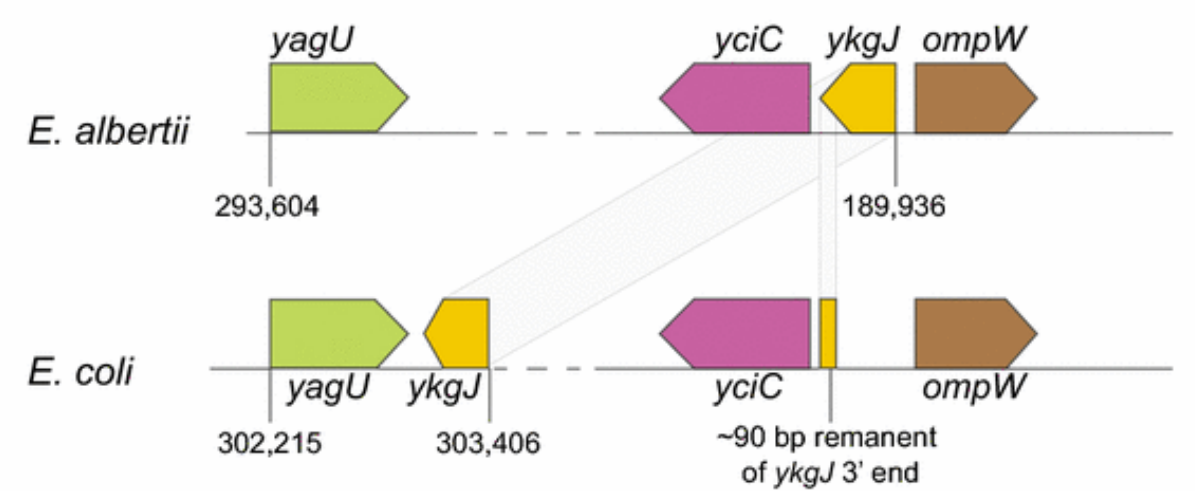

Figure C.5 - EcsR2 evolved in a novel IGR in E. coli. (A) The $y a g U-y k g J$ IGR is present only in E. coli. (B) Relocation of $y \mathrm{kgJ}$ next to $y a g U$ in $E$. coli resulted in the formation of a novel IGR that contains EcsR2. Gene locations based on E. albertii (NZ_CP007025.1) and E. coli (NC_000913.2). 
a

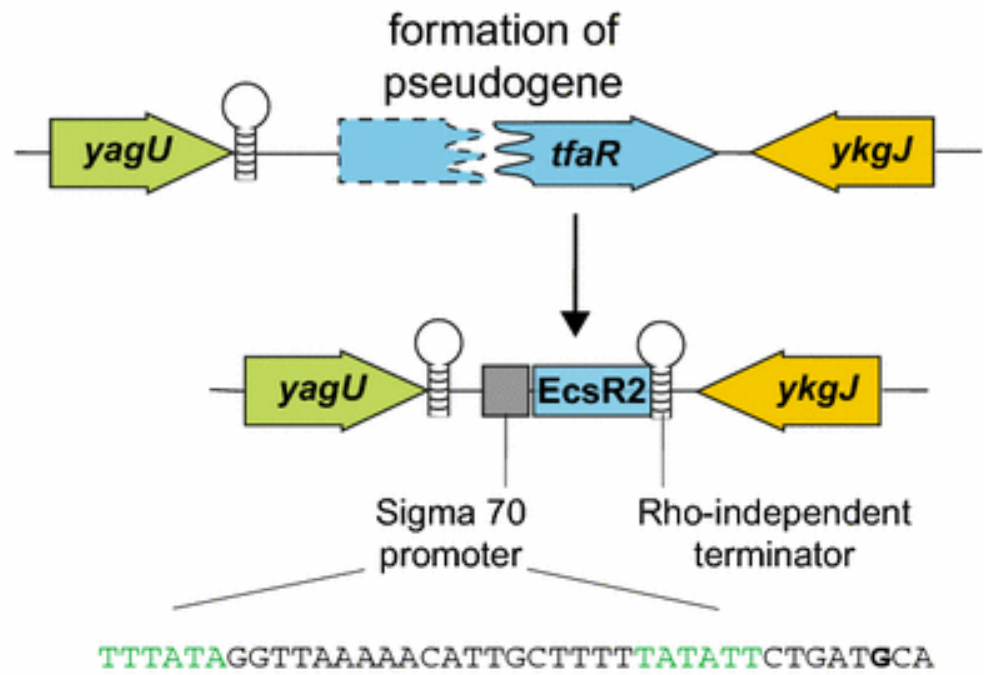

b

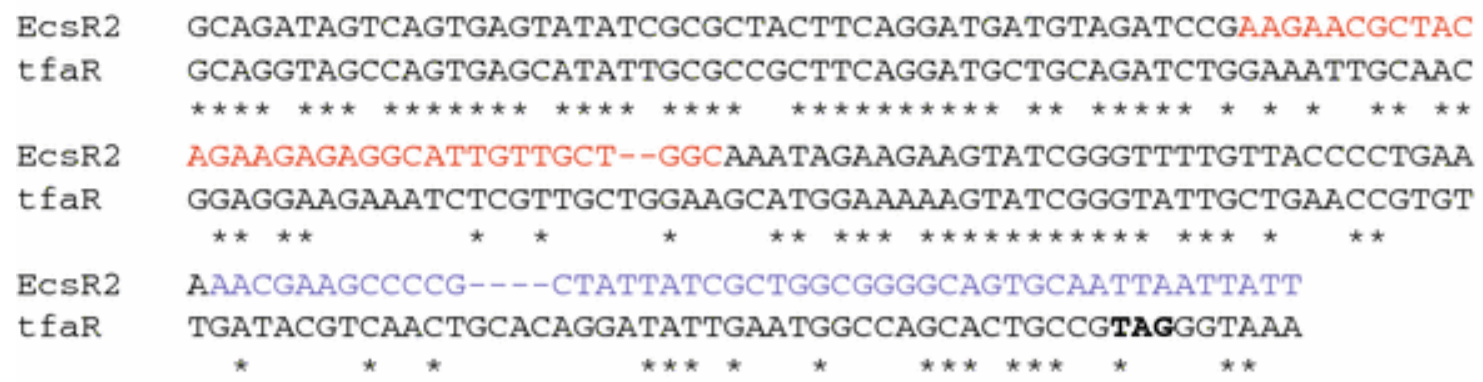

Figure C.6 - Origination of EcsR2 from a degraded prophage gene. (A) A

pseudogenized version of a prophage gene $(t f a R)$ evolved into EcsR2 by gaining a promoter-like sequence and an intrinsic terminator. Putative -10 and -35 sequences are in green, and the transcription start site of EcsR2 is in bold. (B) Alignment of EcsR2 gene and its homologous region in $t f a R$ gene. The putative mRNA-binding region and the intrinsic terminator of EcsR2 are shown in red and purple, respectively. The $t f a R$ stop codon is in bold. The $5^{\prime}$ end of EcsR2 (nucleotides 1-107) has $\sim 75 \%$ identity, whereas the $3^{\prime}$ end (nucleotides 108-166) has $\sim 50 \%$ identity to tfaR. 
cells transiently expressing EcsR2 (cells with empty vector was used as control). This approach has been used previously to identify sRNA targets because pulse expression of sRNA limits indirect regulatory effects (e.g., Zhang et al. 1998; Wang et al. 2015). The RNA-seq analysis identified 26 genes that were significantly downregulated in the EcsR2-expressing strain (Table S2, found in Additional_file_A9.pdf). Further, we combined in vivo RNA crosslinking (Lustig et al. 2010; Liu et al. 2015) with RNA-seq to identify mRNAs that could directly interact with EcsR2 (Fig. S1, found in Additional_file_A9.pdf). This approach (Crosslink-seq) identified nine mRNAs that were potentially bound to EcsR2 (Table S3, found in Additional_file_A9.pdf).

One gene that was identified through both RNA-seq and Crosslink-seq as a potential direct target of EcsR2 was ansB (downregulated $\sim 16$ fold in RNA-seq, and enriched $>3$ fold in Crosslink-seq). In silico modeling predicted that EcsR2 could bind to AnsB mRNA via nucleotides within an unstructured region: positions +52 to +83 (Fig. S2, found in Additional_file_A9.pdf); coincidentally, using a sliding-window analysis that mapped the rate of polymorphism across EcsR2, we identified the same region to be evolving at a much lower rate than the rest of the sRNA (Figure C.7). Previous studies have shown that mRNA-binding sites are the most conserved regions within sRNAs (Peer and Margalit 2011; Richter and Backofen 2012), indicating that the +50 to +80 region is the potential AnsB-binding site. Additionally, although this region is highly conserved among E. coli strains (Figure C.7), it seems to have evolved considerably from its progenitor $t f a R$ gene (Figure C.6, Fig. S3, found in Additional_file_A9.pdf), potentially due to its functional importance. To verify EcsR2's ability to regulate ans $B$ expression, 
a

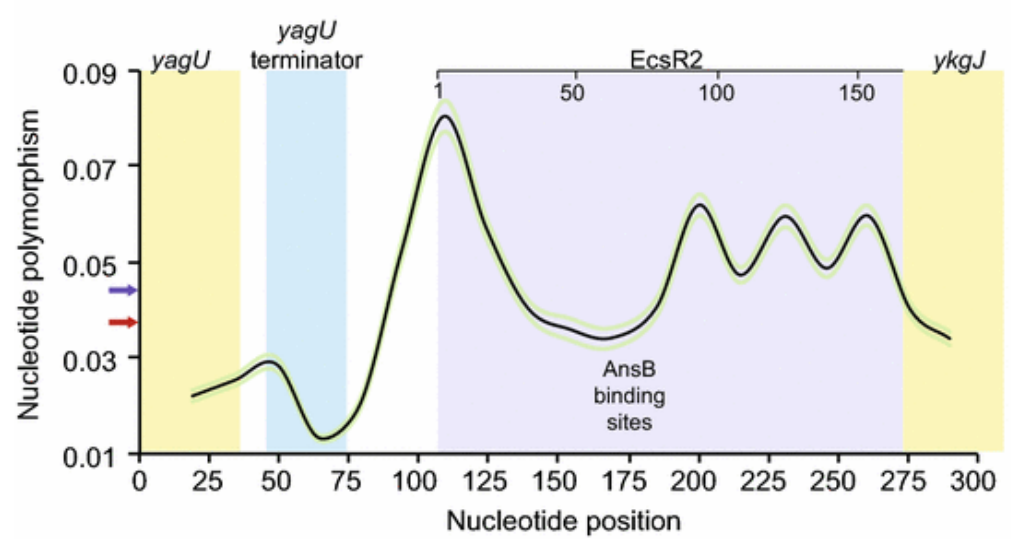

b

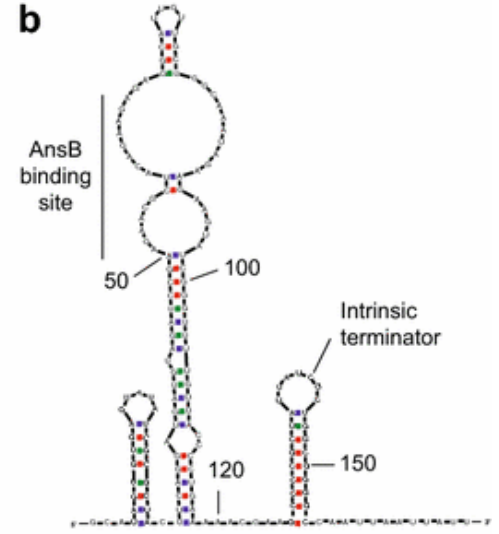

Figure C.7 - EcsR2 sequence conservation and predicted structure. (A) Nucleotide differences calculated for the yagU-ykgJ IGR using a sliding-window analysis is represented by the black line, and the flanking green lines indicate the $95 \%$ confidence interval. The locations of the $3^{\prime}$ ends of $y a g U$ and $y \mathrm{kgJ}$ genes are shown in yellow. The most conserved region (blue) within the IGR correlates with yagU's intrinsic terminator. The location of EcsR2 within the IGR is highlighted in purple. The blue and red arrows on $y$-axis indicate the average nucleotide polymorphism for EcsR2 and IGR, respectively. (B) The predicted secondary structure of EcsR2 was generated using mfold web server. 
we pulse induced the expression of full-length EcsR2 and a version of EcsR2 in which the putative binding site was deleted. We quantified ansB expression using qRT-PCR and observed significant reduction in ans $B$ expression only with the full-length version of EcsR2 (Figure C.8), suggesting that the putative mRNA-binding region is required for gene regulation.

\section{Conserved sRNAs are Under Purifying Selection}

To assess the impact of natural selection on sRNAs, we analyzed a subset of sRNAs $(\mathrm{n}=38)$ that are conserved in E. coli and S. enterica (74 and 102 strains, respectively) (Supplemental dataset 1, found in Additional_file_A13.csv). As expected, younger sRNAs have higher rates of polymorphism and divergence than older sRNAs (Figure C.9). Interestingly, both young and old sRNAs are evolving at significantly lower rates than genome-wide four-fold degenerate sites (proxy for neutral evolution), showing that purifying selection is acting to preserve sRNAs in both bacteria, probably due to their contribution to bacterial fitness, as shown previously for miRNAs in humans, Drosophila, and Caenorhabditis (Quach et al. 2009; Jovelin and Cutter 2014; Lyu et al. 2014). 


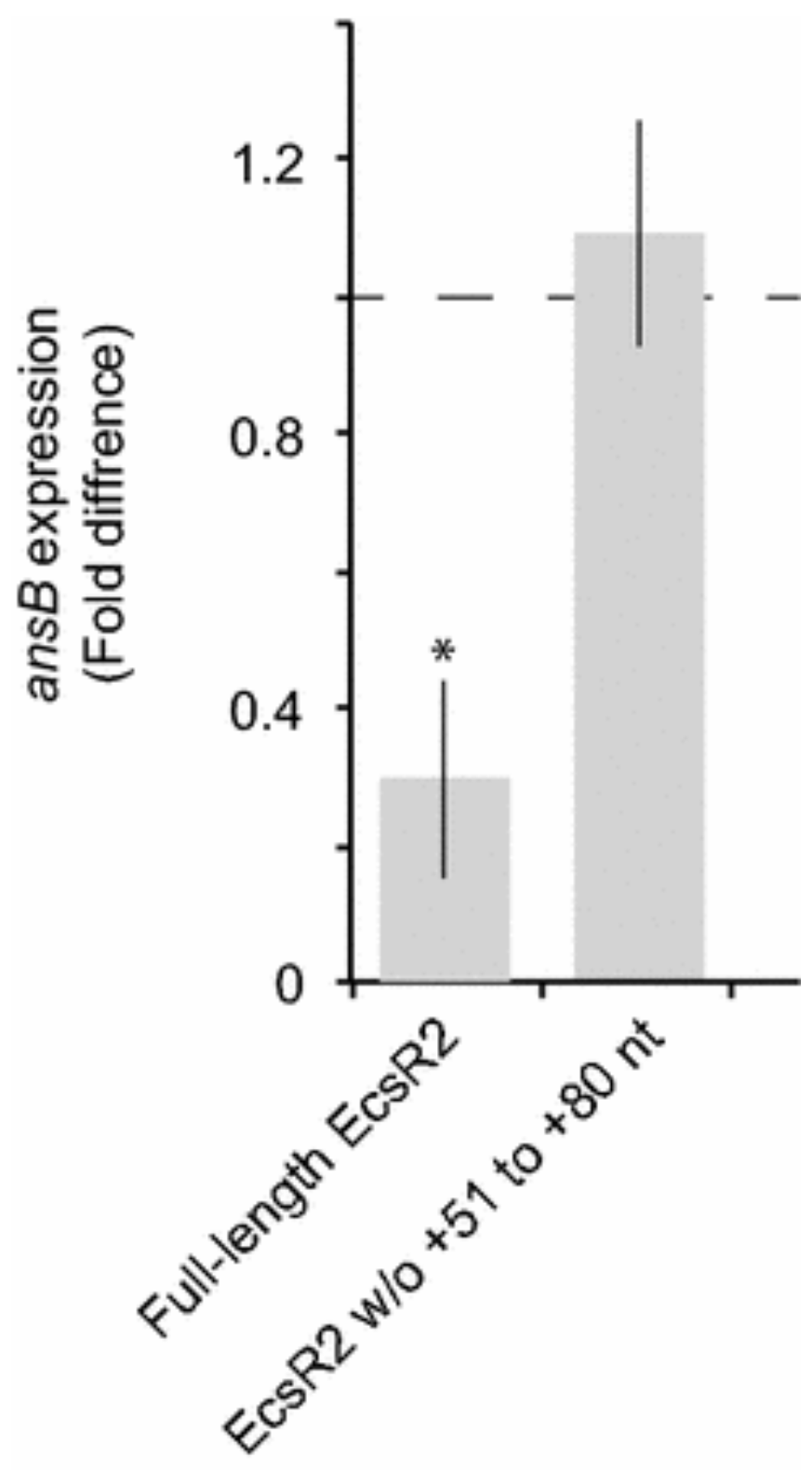

Figure C.8 - EcsR2 downregulates ansB expression. Fold difference in ans $B$ expression in the presence of full-length EcsR2 or EcsR2 without nucleotides +51 to +80 , in comparison to a strain lacking EcsR2 (normalized to 1, dashed line). Data represent the means of three experiments \pm standard deviations. Asterisk indicates $p<0.01$ (unpaired $t$ test). 

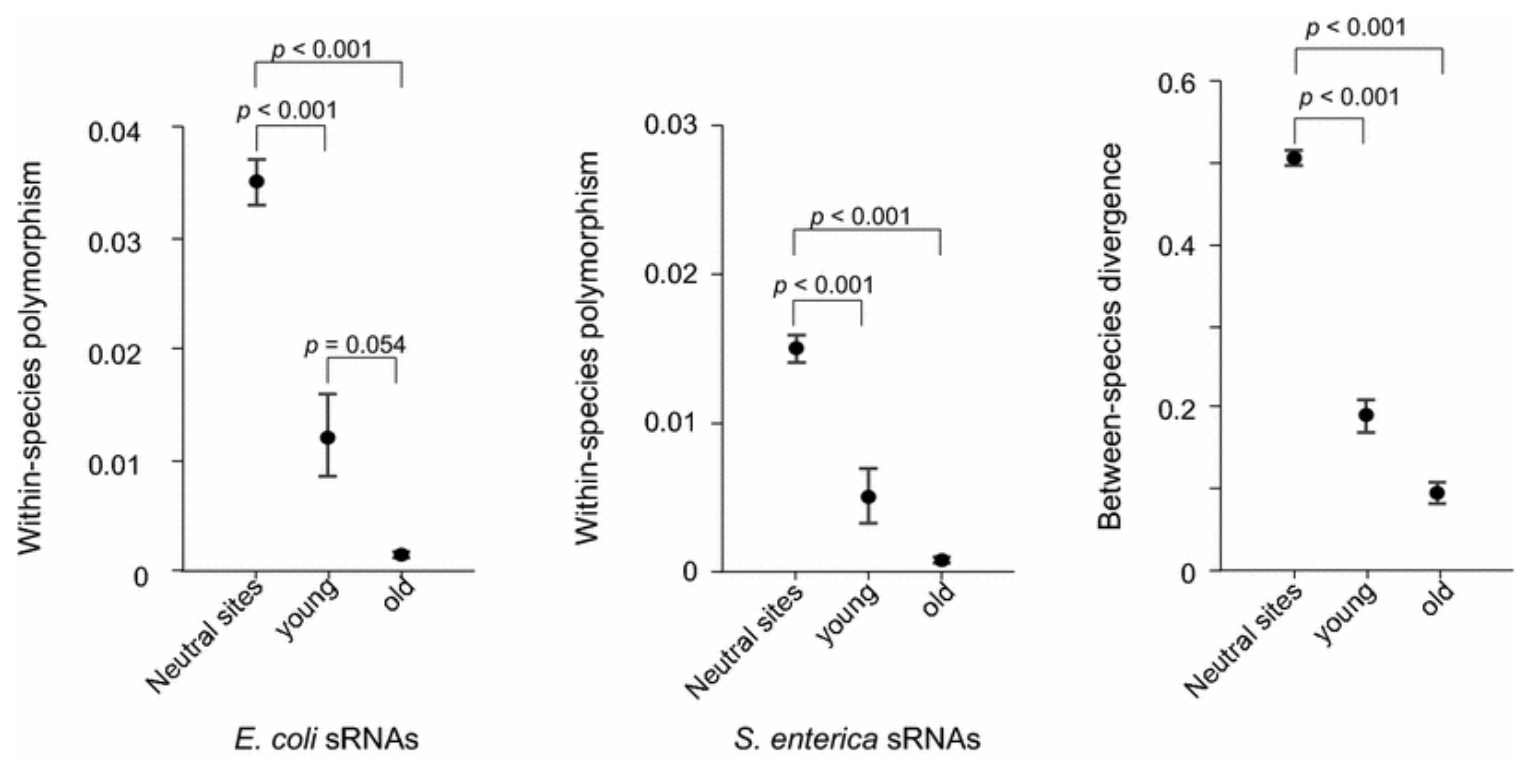

Figure C.9 - sRNAs are under purifying selection. Selective constrains are stronger on sRNAs $(n=38)$ than on neutral sites (4-fold degenerate sites; $n=100)$. sRNAs present in the common ancestor of all six species were considered old and the rest as young. $p$ values calculated using Kruskal-Wallis test and Dunn's test for multiple comparisons. 


\section{C.5 DISCUSSION}

Although sRNAs are critical to gene regulation, we lack a clear understanding of how they originate and evolve in bacteria. In this study, we show that young sRNAs are expressed at low levels and evolve at faster rates than older sRNAs, thereby uncovering a novel process that potentially facilitates the establishment of new sRNAs in bacterial genomes. We also discovered that an sRNA (EcsR2) emerged from a degraded bacteriophage protein-coding gene, thus revealing the first known source for sRNA origination in bacteria. Similar to the origination of EcsR2, new ncRNA genes in eukaryotes have arisen from the remnants of protein-coding genes by gaining regulatory motifs (Kaessmann 2010; Ruiz-Orera et al. 2015). Additionally, in eukaryotes, the evolution of a spurious transcript into a functional ncRNA is associated with changes in the RNA's secondary structure (Heinen et al. 2009). In concordance with this observation, in EcsR2, the putative mRNA-binding region appears to have become more unstructured, whereas the intrinsic terminator likely became more structured (Figure C.6, Fig. S3, found in Additional_file_A9.pdf). Analogous to EcsR2, another E. coli-specific sRNA IsrA (McaS) (Jørgensen et al. 2013) is also evolving at a rapid rate $(\pi=0.050)$, validating the observation that young sRNAs evolve swiftly in bacteria. Most of the other sRNAs with similarly high rates of evolution are antisense RNAs that are part of toxinantitoxin systems (Fozo et al. 2008). Interestingly, SgrS, an sRNA present in several Gammaproteobacteria (Horler and Vanderpool 2009), displayed an elevated rate of evolution $(\pi=0.060)$. However, a closer examination revealed that the sRNA has 
diverged considerably in 30 out of the $85 \mathrm{E}$. coli strains used in our analysis. If we consider only the other 55 genomes, the nucleotide diversity value for SgrS falls within the expected range for older sRNAs $(\pi=0.0029)$. The reasons for the disparity in SgrS evolutionary rates in the two E. coli cohorts are unknown.

Similar to protein-coding genes, most sRNA genes are evolving under purifying selection, suggestive of their importance to bacterial fitness; however, young sRNAs are evolving much more rapidly than evolutionarily older sRNAs, and young sRNAs are expressed at significantly lower levels than established sRNAs. One of the probable causes for the low expression could be that their promoters are not yet fully functional. In bacteria, promoter-like sequences arise spontaneously through point mutations, especially in IGRs (Mendoza-Vargas et al. 2009), and inefficient transcription from these promoters is the main source of pervasive transcripts i.e., RNAs originating from all across the genome (Dornenburg et al. 2010; Raghavan et al. 2012; Thomason et al. 2015). The functions, if any, of these genome-wide transcripts are not yet understood, but they could serve as the raw material for the emergence of new functional RNAs (Gottesman and Storz 2011; Wade and Grainger 2014; Lybecker et al. 2014). Pervasive transcription has been observed in all domains of life, and recently it was shown that new functional RNAs could evolve from such transcripts in humans (Ruiz-Orera et al. 2015). Our data also point towards such a scenario where the emergence of a promoter-like sequence resulted in the production of a transcript that evolved into EcsR2 by gaining regulatory motifs.

To be functional, an sRNA only requires a small seed sequence with partial complementarity to an mRNA; therefore, several such target mRNAs should occur in a 
bacterial genome just through chance. Although a few nascent sRNA-mRNA interactions might have positive outcomes, most are likely deleterious, which could be mitigated by the low expression of incipient sRNAs, while new beneficial interactions could arise through rapid sRNA evolution. Similar to what we show in young enterobacterial sRNAs, low expression and rapid evolution have been observed in young miRNAs (Chen and Rajewsky 2007; Jovelin and Cutter 2014; Lyu et al. 2014), suggesting that this a universal process that facilitates the emergence of new non-coding regulatory RNAs in all domains of life. 


\section{C.6 REFERENCES}

Bernick DL, Dennis PP, Lui LM, Lowe TM. Diversity of antisense and other non-coding RNAs in archaea revealed by comparative small RNA sequencing in four Pyrobaculum species. Front Microbiol. 2012;3:1-18.

Breaker RR. Prospects for riboswitch discovery and analysis. Mol Cell. 2011;43:867879.

Chen K, Rajewsky N. The evolution of gene regulation by transcription factors and microRNAs. Nat Rev Genet. 2007;8:93-103.

Datsenko KA, Wanner BL. One-step inactivation of chromosomal genes in Escherichia coli K-12 using PCR products. Proc Natl Acad Sci. 2000;97:6640-6645.

Dornenburg J, DeVita A, Palumbo M, Wade J. Widespread antisense transcription in Escherichia coli. MBio. 2010;1:e00024.

Fozo EM, Hemm MR, Storz G. Small toxic proteins and the antisense RNAs that repress them. Microbiol Mol Biol Rev. 2008;72:579-589.

Gardner PP, Wilm A, Washietl S. A benchmark of multiple sequence alignment programs upon structural RNAs. Nucleic Acids Res. 2005;33:2433-2439.

Georg J, Hess WR. cis-antisense RNA, another level of gene regulation in bacteria. Microbiol Mol Biol Rev. 2011;75:286-300.

Gottesman S, Storz G. Bacterial small RNA regulators: versatile roles and rapidly evolving variations. Cold Spring Harb Perspect Biol. 2011;3:a003798. 
Gruber AR, Lorenz R, Bernhart SH, et al. The Vienna RNA websuite. Nucleic Acids Res. 2008;36:W70-W74.

Guzman LM, Belin D, Carson MJ, Beckwith J. Tight regulation, modulation, and highlevel expression by vectors containing the arabinose pBAD promoter. J Bacteriol. $1995 ; 177: 4121-4130$.

Heinen TJAJ, Staubach F, Häming D, Tautz D. Emergence of a new gene from an intergenic region. Curr Biol. 2009;19:1527-1531.

Hoeppner MP, Gardner PP, Poole AM. Comparative analysis of RNA families reveals distinct repertoires for each domain of life. PLoS Comput Biol. 2012;8:e1002752. Horler RSP, Vanderpool CK. Homologs of the small RNA SGRS are broadly distributed in enteric bacteria but have diverged in size and sequence. Nucleic Acids Res. $2009 ; 37: 5465-5476$.

Jørgensen MG, Thomason MK, Havelund J, et al. Dual function of the McaS small RNA in controlling biofilm formation. Genes Dev. 2013;27:1132-1145.

Jovelin R, Cutter AD. Microevolution of nematode miRNAs reveals diverse modes of selection. Genome Biol Evol. 2014;6:3049-3063.

Kaessmann H. Origins, evolution, and phenotypic impact of new genes. Genome Res. 2010;20:1313-1326.

Kortmann J, Narberhaus F. Bacterial RNA thermometers: molecular zippers and switches. Nat Rev Microbiol. 2012;10:255-265. 
Kröger C, Colgan A, Srikumar S, et al. An infection-relevant transcriptomic compendium for Salmonella enterica serovar Typhimurium. Cell Host Microbe. 2013;14:683695.

Librado P, Rozas J. DnaSP v5: a software for comprehensive analysis of DNA polymorphism data. Bioinformatics. 2009;25:1451-1452.

Lindgreen S, Umu SU, Lai ASW, et al. Robust identification of noncoding RNA from transcriptomes requires phylogenetically-informed sampling. PLoS Comput Biol. 2014;10:e1003907.

Liu N, Niu G, Xie Z, et al. The Streptococcus mutans irvA gene encodes a trans-acting riboregulatory mRNA. Mol Cell. 2015;57:179-190.

Lustig Y, Wachtel C, Safro M, et al. "RNA walk" a novel approach to study RNA-RNA interactions between a small RNA and its target. Nucleic Acids Res. 2010;38:e5.

Lybecker M, Bilusic I, Raghavan R. Pervasive transcription: detecting functional RNAs in bacteria. Transcription. 2014;5:e944039.

Lyu Y, Shen Y, Li H, et al. New microRNAs in Drosophila-birth, death and cycles of adaptive evolution. PLoS Genet. 2014;10:e1004096.

Mendoza-Vargas A, Olvera L, Olvera M, et al. Genome-wide identification of transcription start sites, promoters and transcription factor binding sites in E. coli. PLoS ONE. 2009;4:e7526.

Michaux C, Verneuil N, Hartke A, Giard J-C. Physiological roles of small RNA molecules. Microbiology. 2014;160:1007-1019. 
Miyakoshi M, Chao Y, Vogel J. Regulatory small RNAs from the $3^{\prime}$ regions of bacterial mRNAs. Curr Opin Microbiol. 2015;24:132-139.

Nawrocki EP, Burge SW, Bateman A, et al. Rfam 12.0: updates to the RNA families database. Nucleic Acids Res. 2015;43:D130-D137.

Nei M. Molecular evolutionary genetics. Columbia University Press, New York, 1987.

Ochman H, Wilson AC. Evolution in bacteria: evidence for a universal substitution rate in cellular genomes. J Mol Evol. 1987;26:74-86.

Peer A, Margalit H. Accessibility and evolutionary conservation mark bacterial smallRNA target-binding regions. J Bacteriol. 2011;193:1690-1701.

Peer A, Margalit H. Evolutionary patterns of Escherichia coli small RNAs and their regulatory interactions. RNA. 2014;20:994-1003.

Quach H, Barreiro LB, Laval G, et al. Signatures of purifying and local positive selection in human miRNAs. Am J Hum Genet. 2009;84:316-327.

Raghavan R, Groisman EA, Ochman H. Genome-wide detection of novel regulatory RNAs in E. coli. Nucleic Acids Res. 2011;10:1487-1497.

Raghavan R, Sloan DB, Ochman H. Antisense transcription is pervasive but rarely conserved in enteric bacteria. MBio. 2012;3:e00156.

Raghavan R, Kacharia FR, Millar JA, et al. Genome rearrangements can make and break small RNA genes. Genome Biol Evol. 2015;7:557-566.

Richter AS, Backofen R. Accessibility and conservation: general features of bacterial small RNA-mRNA interactions? RNA Biol. 2012;9:954-965. 
Ruiz-Orera J, Hernandez-Rodriguez J, Chiva C, et al. Origins of de novo genes in human and chimpanzee. PLoS Genet. 2015;11:1-24.

Sievers F, Wilm A, Dineen D, et al. Fast, scalable generation of high-quality protein multiple sequence alignments using Clustal Omega. Mol Syst Biol. 2011;7:539.

Skippington E, Ragan MA. Evolutionary dynamics of small RNAs in 27 Escherichia coli and Shigella genomes. Genome Biol Evol. 2012;4:330-345.

Storz G, Vogel J, Wassarman KM. Regulation by small RNAs in bacteria: expanding frontiers. Mol Cell. 2011;43:880-891.

Thomason M, Storz G. Bacterial antisense RNAs: how many are there and what are they doing? Annu Rev Genet. 2010;44:167-188.

Thomason MK, Bischler T, Eisenbart SK, et al. Global transcriptional start site mapping using differential RNA sequencing reveals novel antisense RNAs in Escherichia coli. J Bacteriol. 2015;197:18-28.

Updegrove TB, Shabalina SA, Storz G. How do base-pairing small RNAs evolve? FEMS Microbiol Rev. 2015;39:379-391.

Wade JT, Grainger DC. Pervasive transcription: illuminating the dark matter of bacterial transcriptomes. Nat Rev Microbiol. 2014;12:647-653.

Wang J, Rennie W, Liu C, et al. Identification of bacterial sRNA regulatory targets using ribosome profiling. Nucleic Acids Res. 2015;43:10308-10320.

Wilson RC, Doudna J. Molecular mechanisms of RNA interference. Annu Rev Biophys. $2013 ; 42: 217-239$. 
Wright PR, Georg J, Mann M, et al. CopraRNA and IntaRNA: predicting small RNA targets, networks and interaction domains. Nucleic Acids Res. 2014;42:W119W123.

Zhang A, Altuvia S, Tiwari A, et al. The OxyS regulatory RNA represses rpoS translation and binds the Hfq (HF-I) protein. EMBO J. 1998;17:6061-6068.

Zuker M. Mfold web server for nucleic acid folding and hybridization prediction. Nucleic Acids Res. 2003;31:3406-3415. 


\begin{abstract}
APPENDIX D
Accumulation and expression of multiple antibiotic resistance genes in Arcobacter cryaerophilus that thrives in sewage
\end{abstract}

\title{
D.1 ABSTRACT
}

We explored the bacterial diversity of untreated sewage influent samples of a wastewater treatment plant in Tucson, AZ and discovered that Arcobacter cryaerophilus, an emerging human pathogen of animal origin, was the most dominant bacterium. The other highly prevalent bacteria were members of the phyla Bacteroidetes and Firmicutes, which are major constituents of human gut microbiome, indicating that bacteria of human and animal origin intermingle in sewage. By assembling a near-complete genome of $A$. cryaerophilus, we show that the bacterium has accumulated a large number of antibiotic resistance genes (ARGs) probably enabling it to thrive in the wastewater. We also determined that a majority of ARGs was being expressed in sewage, suggestive of trace levels of antibiotics or other stresses that could act as a selective force that amplifies multidrug resistant bacteria in municipal sewage. Because all bacteria are not eliminated even after several rounds of wastewater treatment, ARGs in sewage could affect public health due to their potential to contaminate environmental water. 


\section{D.2 INTRODUCTION}

Non-coding Over the past few decades, based on numerous studies that examined the bacterial composition of wastewater during varying stages of treatment, there is growing evidence that sewage is an important hub for horizontal gene transfer (HGT) of antibiotic resistance genes (e.g., Baquero, Martínez \& Cantón, 2008; Zhang, Shao \& Ye, 2012; Rizzo et al., 2013; Pehrsson et al., 2016). Additionally, studies have shown that discharge of treated sewage allows these concentrated communities to spread into environmental water (Okoh et al., 2007; Varela \& Manaia, 2013). The Arcobacter genus is commonly detected in sewage treatment plants around the world (Collado et al., 2008; Zhang, Shao \& Ye, 2012; Varela \& Manaia, 2013). This sparsely studied Epsilonproteobacteria is frequently associated with veterinary diseases, and is closely related to Campylobacter, and is considered an emerging human pathogen that causes enteritis and bacteremia (Kabeya et al., 2004; Morita et al., 2004; Collado et al., 2008). In addition, Arcobacter is known to be resistant to a wide array of commonly used antibiotics, with varying resistance profiles observed in different species (Houf et al., 2004; Abay et al., 2012; Rahimi, 2014), but the genes that enable antibiotic resistance are mostly unknown (Abdelbaqi et al., 2007; Miller et al., 2007).

In this study, we examined the bacterial diversity and the presence and expression of antibiotic resistance genes (ARGs) and virulence factors in untreated sewage. Our analyses revealed that an A. cryaerophilus strain that contained multiple ARGs was a major constituent of the sewage microbiome. In addition, we detected a large number of 
expressed ARGs and virulence factors in A. cryaerophilus and in the rest of the sewage microbiome, which portends potential public health risk if bacteria carrying these genes contaminate public water resources. 


\section{D.3 MATERIALS AND METHODS}

\section{Sewage sample collection, DNA and RNA extraction, and deep-sequencing}

Three untreated sewage influent samples $(50 \mathrm{ml})$ were collected at the Roger Road Wastewater Reclamation Facility, Tucson, Arizona (March 2012) and immediately transferred on ice to the laboratory and stored at $-80^{\circ} \mathrm{C}$ until further use. The sewage samples were spun down $\left(12,000 \times \mathrm{G}, 15 \mathrm{~min}, 4^{\circ} \mathrm{C}\right)$ and the pellets were suspended in 1 $\mathrm{ml}$ of TRI reagent (Life Technologies). Total RNA from each sample was extracted from the aqueous phase and corresponding DNA was isolated from the interphase using a protocol provided by the manufacturer. RNA samples were treated with TurboDNAse (Life Technologies) to remove contaminating DNA, and PCR reactions using 16S rDNA primers were performed to confirm complete DNA removal. Furthermore, RNA samples were depleted of ribosomal RNA using RiboZero Bacteria and RiboZero Human kits (Illumina). Around $100 \eta \mathrm{g}$ of RNA from each sample was used to prepare directional mRNA-seq libraries using the Illumina Small RNA Sample Preparation Kit and Directional mRNA-seq Sample Preparation protocol provided by Illumina Inc. DNA samples were further purified using DNeasy kit (Qiagen) and around $5 \mu \mathrm{g}$ of DNA from each sample was used to prepare paired-end DNA-seq libraries using the Paired-End sample Preparation Kit (Illumina). All RNA libraries were pooled into a single lane, and all DNA samples were pooled into another lane of Illumina HiSeq 2000 and were sequenced at the Yale Center for Genome Analysis (RNA-seq: single-end, 75 cycles; DNA-seq: paired end, $2 \times 75$ cycles) using standard adapters. All DNA and RNA reads 
have been deposited at NCBI (BioProject PRJNA354077).

\section{Taxonomic classification}

DNA reads were cleaned by removing adapters and were filtered by quality $(\geq Q 20)$ and length ( $\geq 50 \mathrm{bp}$ ) using Trimmomatic v0.32 (Bolger, Lohse \& Usadel, 2014). Each library was assembled into contigs using 17,000,000 reads and IDBA-UD (Peng et al., 2012), and normalized to the smallest library size $(6,000$ contigs $)$ using Seqtk (https://github.com/lh3/seqtk) (Perner et al., 2014) (Table S1, found in Additional_file_A14.pdf). All contigs were searched against the NCBI nt database using BLAST and analyzed in MEGAN (Huson et al., 2007), requiring at least $70 \%$ of the query sequence to align with the subject sequence with $\geq 70 \%$ identity to be assigned to a given phylum. Contigs classified at the phylum level (48\%, 57\%, and 54\% of contigs from the three samples, respectively) were used to determine their detailed taxonomic positions. Remaining contigs either did not have significant BLAST hits or mapped to unidentified environmental samples; however, all contigs were used in downstream analyses (detection of antibiotic resistance genes, virulence factors etc.).

\section{Identification of antibiotic resistance genes, virulence factors, transposases, and bacteriophage genes}

Contigs were run through MetaProdigal (Hyatt et al., 2012) to identify encoded ORFs, which were annotated by mapping to antibiotic resistance genes, virulence factors, bacterial transposases, and prophages obtained respectively from CARD, PATRIC, 
InterPro, and PHAST databases using PHMMER, with an $E$-value of at least $1 \mathrm{e}-10$ as the cutoff (Finn, Clements \& Eddy, 2011; Zhou et al., 2011; McArthur et al., 2013; Wattam et al., 2014; Mitchell et al., 2015). RNA reads from each sample were filtered by quality ( $\geq$ Q20) and length ( $\geq 50$ bp) using Trimmomatic v0.32 and normalized using Seqtk to 50,000,000 reads. They were mapped to annotated ORFs using CLC Genomic Workbench v6.5. A strict mapping criterion (at least 95\% of each read should map with at least $95 \%$ identity to the mapped region) was used in order to minimize non-specific mapping. Genes were filtered and considered expressed based on at least 10 reads mapping to each ORF. Statistical analysis was conducted using SAS Studio v3.4 (SAS Institute, Cary NC).

\section{Arcobacter genome assembly}

DNA reads from all three samples were pooled to gain enough coverage depth, and were assembled into contigs using IDBA-UD (Peng et al., 2012). All contigs were searched against the NCBI nt database using BLASTN and analyzed in MEGAN (Huson et al., 2007). All Arcobacter gene sequences were downloaded from NCBI, and using PHMMER, Arcobacter contigs present in our data were identified with at least $1 \mathrm{e}-10 \mathrm{E}$ value as the cutoff. These contigs were extracted and run through the differential coverage binning procedure for metagenomic data, as described previously (Albertsen et al., 2013). In brief, contigs were binned based on coverage, tetranucleotide frequency, $\mathrm{GC} \%$, and length, then examined for presence of essential single copy genes. Phylogenetic analyses were conducted on nucleotide sequences using several 
housekeeping genes to identify the bins containing $A$. cryaerophilus (marked in blue in Fig. S1, found in Additional_file_A14.pdf). One genome bin with $200 \times$ coverage that contained all A. cryaerophilus housekeeping genes was selected for secondary refinement and finishing (top right cluster in Fig. S2, found in Additional_file_A14.pdf). This cluster of contigs was isolated and all original trimmed DNA reads were mapped against them using Bowtie2 v2.1.0 (Langmead \& Salzberg, 2012). All mapped reads were reassembled into contigs using IDBA-UD. These contigs were combined with all original trimmed DNA reads for scaffold extension using SSPACE (Boetzer et al., 2011) into a final scaffold of $\sim 1.8 \mathrm{Mb}$ over 456 contigs. To check for completeness of the assembled A. cryaerophilus genome, we used a single-copy gene database (Albertsen et al., 2013), and as a control we performed the same analysis with the $A$. butzleri (CP000361.1) genome. Visual representation of draft genome was created using Circos (Krzywinski et al., 2009). The draft genome has been deposited at NCBI under the accession LNTC00000000.

\section{Detection of HGT}

Horizontally acquired genes were identified using HGTector (Zhu, Kosoy \& Dittmar, 2014). Arcobacter was set as self-group, and Campylobacterales was set as exclusion group. This method captured HGT events where only Arcobacter has acquired a particular gene from outside of Campylobacterales and ignored any events where the genes could also have been transferred elsewhere within the order. This conservative approach was used due to the dearth of annotated genomes within Campylobacterales. 
BLASTN parameter thresholds were set at $70 \%$ identity and an $E$-value of at least $1 \mathrm{e}-5$. Several putative HGT genes were examined using phylogenetic analysis to validate the HGTector data (Fig. S3, found in Additional_file_A14.pdf).

\section{Phylogenetic analysis}

Nucleotide sequence alignment for all trees was performed using Clustal Omega (Sievers et al., 2011), and ambiguously aligned regions were removed using Gblocks (Talavera \& Castresana, 2007). The evolution model GTR $+\mathrm{I}+\mathrm{G}$ (General Time Reversible plus Invariant sites plus Gamma distribution) used for all trees was selected using jModelTest2 (Darriba et al., 2012). Bayesian trees were constructed using MrBayes as implemented in Geneious (Huelsenbeck \& Ronquist, 2001; Kearse et al., 2012). A chain length of $1,000,000$ was used with a burn-in fraction of $25 \%$ and sampling every 100 trees. Maximum Likelihood trees were constructed using RAxML (Stamatakis, Hoover \& Rougemont, 2008) as implemented in Geneious with 1,000 bootstrap replicates to confirm Bayesian topologies. Helicobacter pylori (AJ558222.1) was used to root all phylogenetic trees. 


\section{D.4 RESULTS AND DISCUSSION}

\section{A. cryaerophilus thrives in sewage}

For the three sewage samples, taxonomic labels were assigned to at least the phylum level for all contigs with significant BLAST hits. There was no significant difference in bacterial distribution between the three samples (Figure D.1); hence, average values are presented hereafter. Members of the phylum Proteobacteria (67\% of total hits) was the most prevalent bacteria, followed by Bacteroidetes (23\%) and Firmicutes $(9 \%)$. A more comprehensive study that examined several sewage samples from across the USA observed a similar pattern of bacterial phyla abundance (Shanks et al., 2013). However, at the genus level, Arcobacter (an Epsilonproteobacteria) was the most dominant bacterium in our study, making up 39\% of all annotated contigs, unlike members of Gammaproteobacteria (38\% of all pyrotags) in the previous study (Shanks et al., 2013).

Arcobacter is commonly associated with both humans and farm animals (Collado et al., 2008), the latter perhaps more relevant to this specific wastewater treatment plant because agriculture accounts for the largest use of water at around $70 \%$ of all water demand within the state of Arizona (ADWR, 2009). In addition, in Tucson, the wastewater treatment plant served both agricultural and municipal areas (PAG, 2006). Members of the phyla Bacteroidetes and Firmicutes, two of the most abundant bacteria in human gut, were also abundant in the sewage samples (Cho \& Blaser, 2012; Jandhyala et al., 2015). Taken together, these data highlight the important role that sewage systems play as an arena where bacteria of human and animal origin interact, which could 


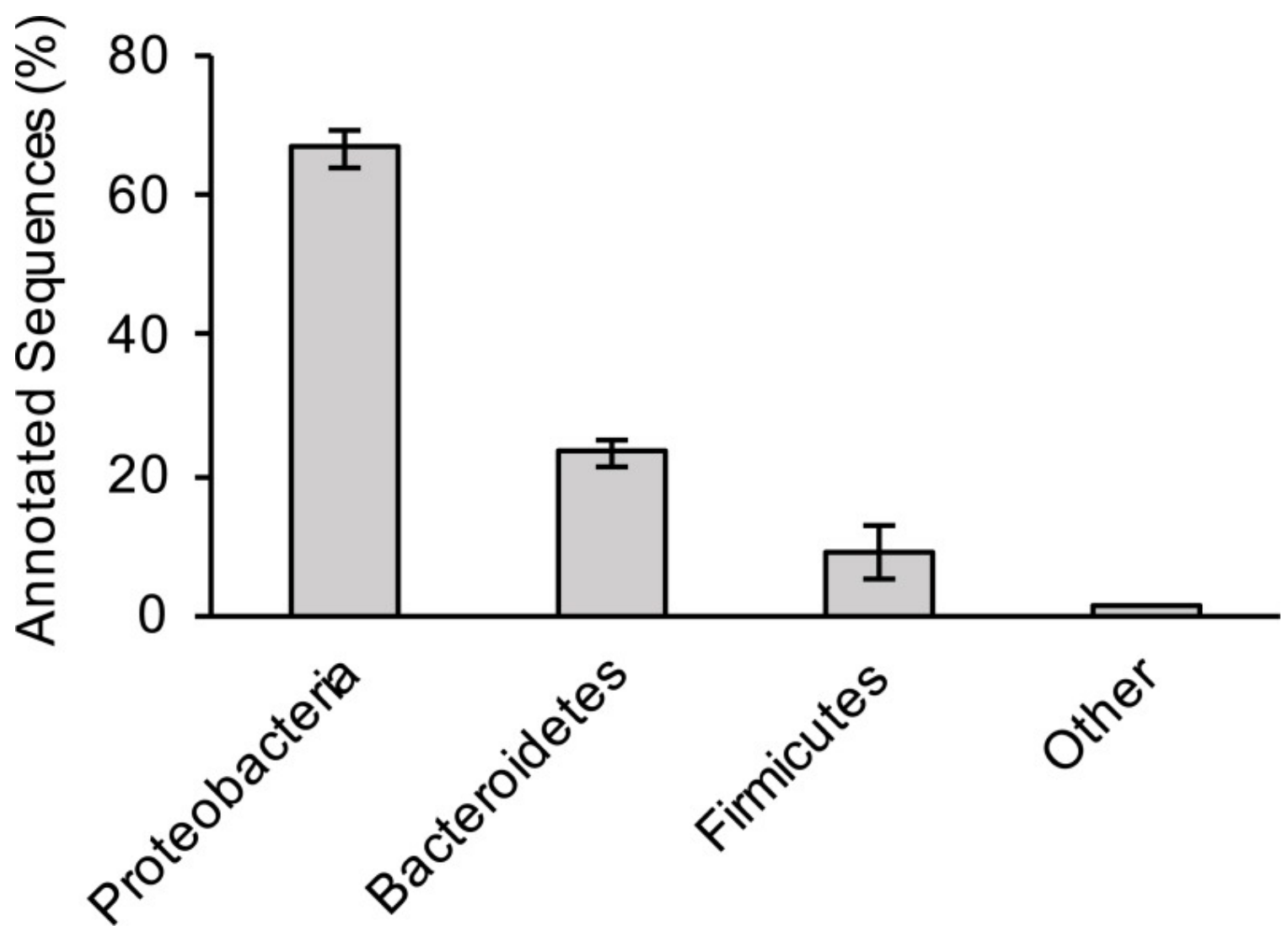

Figure D.1 - Bacterial composition of sewage samples. Percentage of sewage contigs that were assigned to each bacterial phylum is shown. Data represent means of three samples \pm standard deviations. 
promote the exchange of genes between the two groups (Baquero, Martínez \& Cantón, 2008; Gaze et al., 2013; Rizzo et al., 2013). After conducting genomic binning of the Arcobacter contigs utilizing various factors such as coverage depth, $\mathrm{GC} \%$, and tetranucleotide frequency (Albertsen et al., 2013), we were able to identify $\sim 80 \%$ of these contigs as belonging to A. cryaerophilus, an emerging human pathogen that is commonly associated with diseases such as bovine reproductive disorders, diarrhea and hemorrhagic colitis in cattle and sheep (Schroeder-Tucker et al., 1996; Ho, Lipman \& Gaastra, 2006).

\section{Presence and expression of multiple ARGs in A. cryaerophilus}

To better characterize A. cryaerophilus, we assembled a near-complete genome from the DNA-seq reads (Figure D.2). Based on the presence of 100 out of 106 single copy genes (Albertsen et al., 2013) with zero redundant copies, we estimate that the A. cryaerophilus genome is $\sim 95 \%$ complete and contains 2,419 ORFs (including partial genes at the ends of contigs) (Table S2, found in Additional_file_A14.pdf). Among these ORFs, 115 (5\% of ORFs) encode antibiotic resistance genes (ARGs) belonging to 25 categories as

defined by the CARD database (Table S3, found in Additional_file_A14.pdf) (McArthur et al., 2013). Macrolide resistance made up the majority of annotated ARGs (26, 23\%,) (Table S3, found in Additional_file_A14.pdf), with fluoroquinolones (18, 16\%), aminocoumarin $(17,15 \%)$ and vancomycin $(13,11 \%)$ resistance genes being the next largest groups. Because gene expression is a good representation for functional gene activity, we analyzed the expression of $A$. cryaerophilus genes using RNA-seq and discovered that all 115 putative ARGs genes were being expressed (Figure D.3A; 


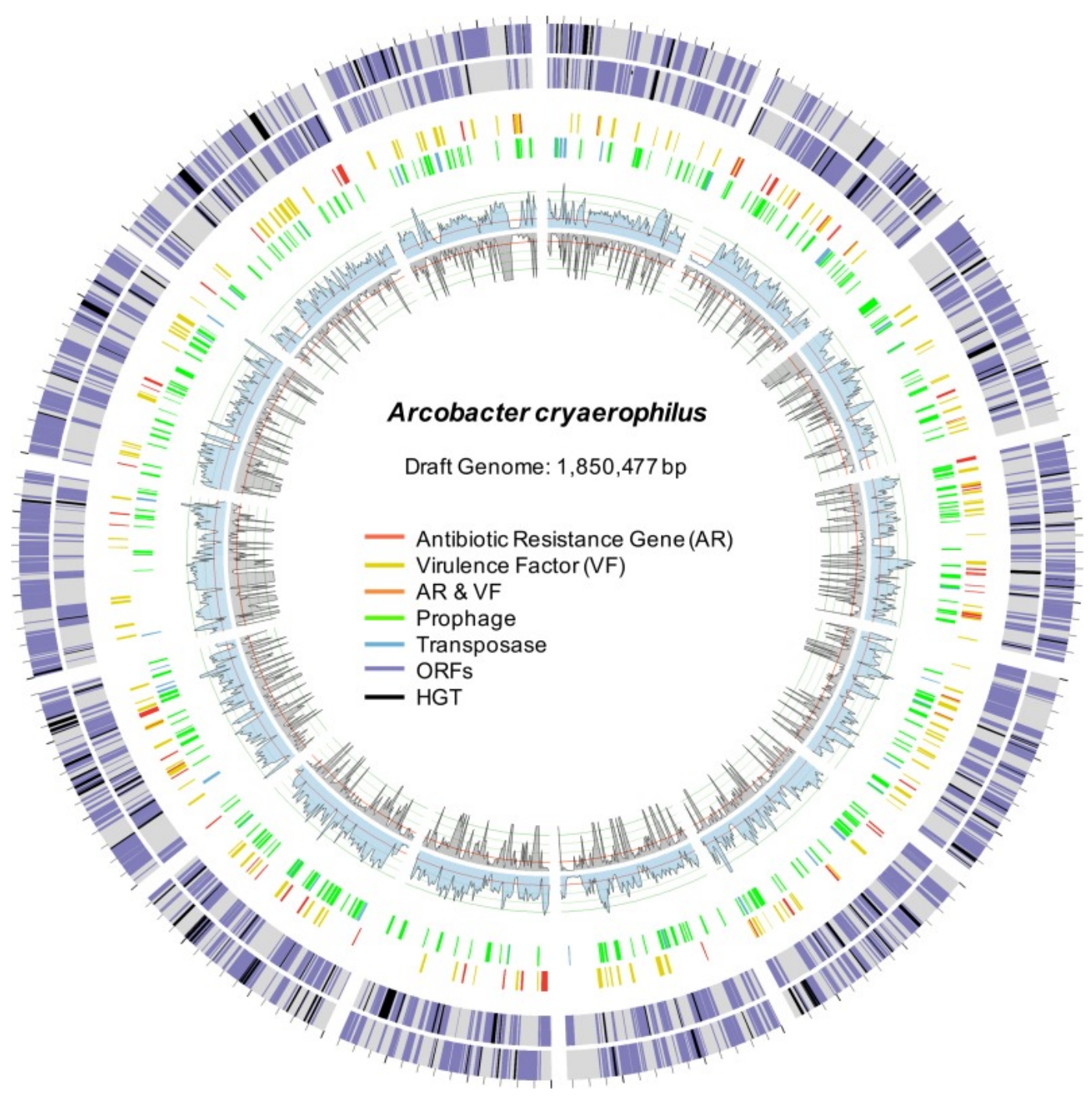

Figure D.2 - Draft genome of $\boldsymbol{A}$. cryaerophilus. Two outer rings show ORFs (purple) on forward and reverse strands, respectively. Black blocks represent horizontally acquired genes. Each tick mark represents 10,000 bp. Middle two rings show positions of features annotated in the center. Inner blue and grey rings show DNA-seq coverage (mean of three samples) and RNA-seq transcription levels (mean of three samples), respectively. Note that 456 original contigs were randomly assigned to 14 equal fragments for easy visualization. 
(A)

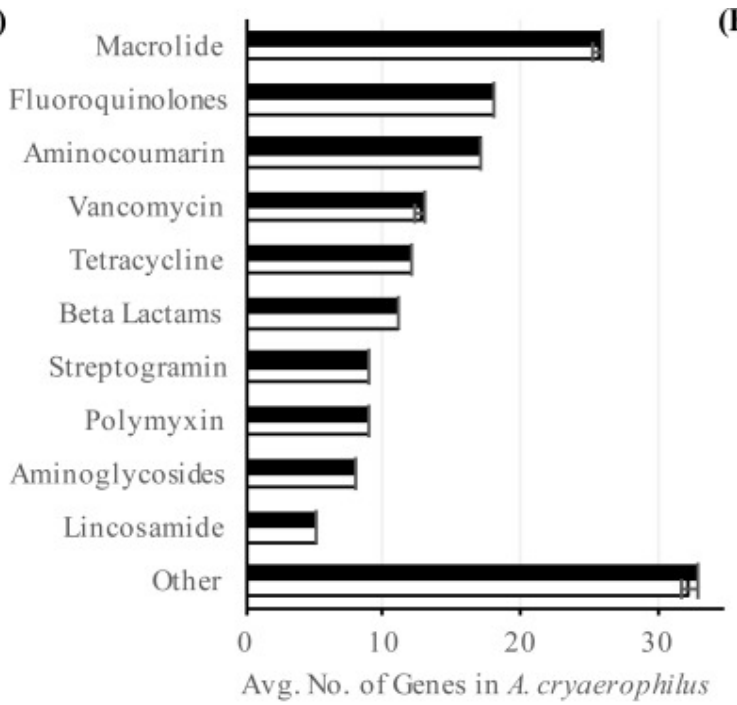

(B)

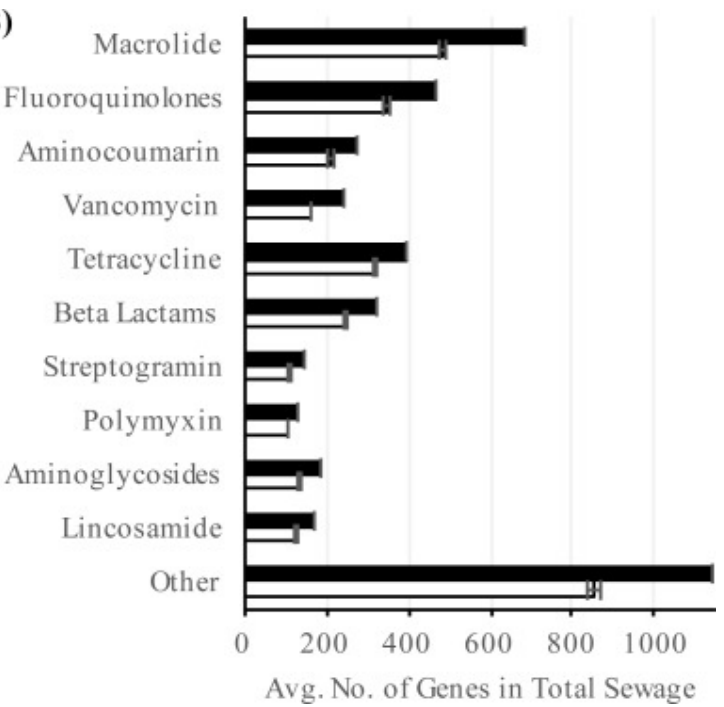

Figure D.3 - Abundance of antibiotic resistance genes. Number of annotated (black) and expressed (white) antibiotic resistance categories in (A) A. cryaerophilus and (B) total sewage sample is shown. Data represent means of three samples \pm standard deviations. 
Additional_file_A15.csv). In comparison, Helicobacter pylori, a closely related Epsilonproteobacteria contain 59 ARGs (4\% of genes); however, in both bacteria around $50 \%$ of ARGs consisted of efflux pumps (Paulsen, Sliwinski \& Saier, 1998). It should be noted that although all ARGs were found to be expressed in A. cryaerophilus, the median level of expression of single copy genes ( 3,887 reads mapped) was found to be $10 \times$ higher than the median level of expression of ARGs (363 reads mapped), probably because higher expression of many ARGs requires strong induction.

To determine the prevalence of ARGs in the total sewage, we extended our analysis to all contigs assembled in our study. Out of the 60,723 ORFs encoded in the sewage contigs, 2,606 ORFs matched 42 antibiotic resistance categories (Table S4, found in Additional_file_A14.pdf). Using RNA-seq, we determined that 2,106 (81\%) of these ORFs were expressed (Figure D.3B). Of the 2,106 putative antibiotic resistance genes expressed in the sewage samples, macrolide resistance genes made up the largest portion $(538,26 \%)$ (Table S4, found in Additional_file_A14.pdf). The next two largest groups were fluoroquinolone resistance $(378,18 \%)$ and tetracycline resistance $(339,16 \%)$ genes. The expression of antibiotic resistance genes could be due to the presence of numerous antibiotics in urban wastewater (Heberer, 2002; Rizzo et al., 2013), which could select for multidrug resistant bacteria, thereby aggravating an already dire situation (Baquero, Martínez \& Cantón, 2008; Rizzo et al., 2013; Wellington et al., 2013; Amos et al., 2014)). It is also possible that ARGs were being expressed constitutively or in response to stress (Poole, 2012). Additionally, previous studies have shown that ARGs are expressed in a wide variety of environments even in the absence of known anthropogenic 
antibiotic pressure (Udikovic-Kolic et al., 2014; Versluis et al., 2015; Noyes et al., 2016); hence, further study is required to determine the stimuli for the observed ARG expression. Interestingly, although the total sewage contigs contained $23 \times$ more ARGs than in $A$. cryaerophilus contigs (2,606 vs. 115$), 44 \%$ of DNA reads mapped to $A$. cryaerophilus ARGs, indicating that while the sewage contained high diversity of ARGs, most non-A. cryaerophilus ARGs were of low abundance.

\section{Signatures of HGT in A. cryaerophilus genome}

We compared our draft genome of A. cryaerophilus to the published genome of $A$. butzleri (CP000361.1), a closely related human and animal pathogen that has been studied much more extensively than A. cryaerophilus (Vandenberg et al., 2004; Miller et al., 2007; Collado et al., 2008) (Fig. S4, found in Additional_file_A14.pdf). As observed previously in other members of this genus (Karadas et al., 2013; Merga et al., 2013), the two Arcobacter species only shared 1,337 genes ( 50\%) (Table D.1,

Additional_file_A16.csv). A comparison of the two genomes was also conducted using RAST (Overbeek et al., 2014), which showed that merely 846 genes with known functions were shared between $A$. butzleri and A. cryaerophilus (Additional_file_A17.csv). This sizable variation in gene content between the two species indicates that HGT could have played a prominent role in shaping the genomes of Arcobacter species. Concomitantly, even after using a very conservative threshold, we detected $209(9 \%)$ and $228(10 \%)$ horizontally acquired genes in A. cryaerophilus and $A$. butzleri, respectively (Table D.1). While similar in scale, only 73 HGT-origin genes were 
Table D.1 - Comparison of Arcobacter genomes.

\begin{tabular}{llll}
\hline Features & A. cryaerophilus & A. butzleri & Overlap \\
\hline Total ORFs & a & 2,259 & 1,337 \\
Horizontally Acquired ORFs & 209 & 228 & 73 \\
Antibiotic Resistance & & & \\
$\quad$ Categories & 25 & 29 & 23 \\
$\quad$ Genes & 115 & 140 & 54 \\
Virulence Factors & & & \\
$\quad$ Categories & 24 & 24 & 22 \\
$\quad$ Genes & 232 & 185 & 92 \\
Transposases & 7 & 7 & 5 \\
$\quad$ Categories & 61 & 57 & 15 \\
$\quad$ Genes & & & \\
Prophages & 290 & 320 & 173 \\
$\quad$ Genes & LNTC00000000 & CP000361.1 & \\
GenBank Accession & & & \\
\hline
\end{tabular}


shared between the two genomes, indicating that parallel HGT events have molded the genomes of the two Arcobacter species.

HGT is known to promote ARG dissemination between bacteria (Hawkey \& Jones, 2009; Gaze et al., 2013; Pehrsson et al., 2016); hence, we compared ARGs present in A. cryaerophilus to those present in A. butzleri in order to identify those that are of possible HGT origin. We identified 140 putative genes belonging to 29 antibiotic resistance categories in $A$. butzleri, and out of the 25 antibiotic resistance categories present in A. cryaerophilus, 23 were present in A. butzleri, with two categories (Glycylcycline and Roxithromycin resistance) found only in A. cryaerophilus, and six categories (Bicyclomycin, Elfamycin, Isoniazid, Kanamycin, Streptomycin, and Teicoplanin resistance) exclusive to A. butzleri (Table D.1). However, within each category large differences in gene content was observed between the two bacteria, with only 54 genes shared between $A$. cryaerophilus and A. butzleri. These data show that even though the antibiotic resistance capabilities of both bacteria overlap, their respective gene repertoires were largely assembled through independent HGT events. Transposons and bacteriophages are important agents of HGT in bacteria, and we found several transposases and bacteriophage ORFs in A. cryaerophilus (61 transposase ORFs, 290 phage ORFs) and A. butzleri $(57,320)$ (Figure D.2 and Table D.1, Table S2, found in Additional_file_A14.pdf). Additionally, we discovered that several of the ARGs in $A$. cryaerophilus were located in close proximity to transposases or bacteriophage genes (Figure D.4), suggestive of a role for these mobile genetic elements in the accumulation of ARGs in this pathogen. 


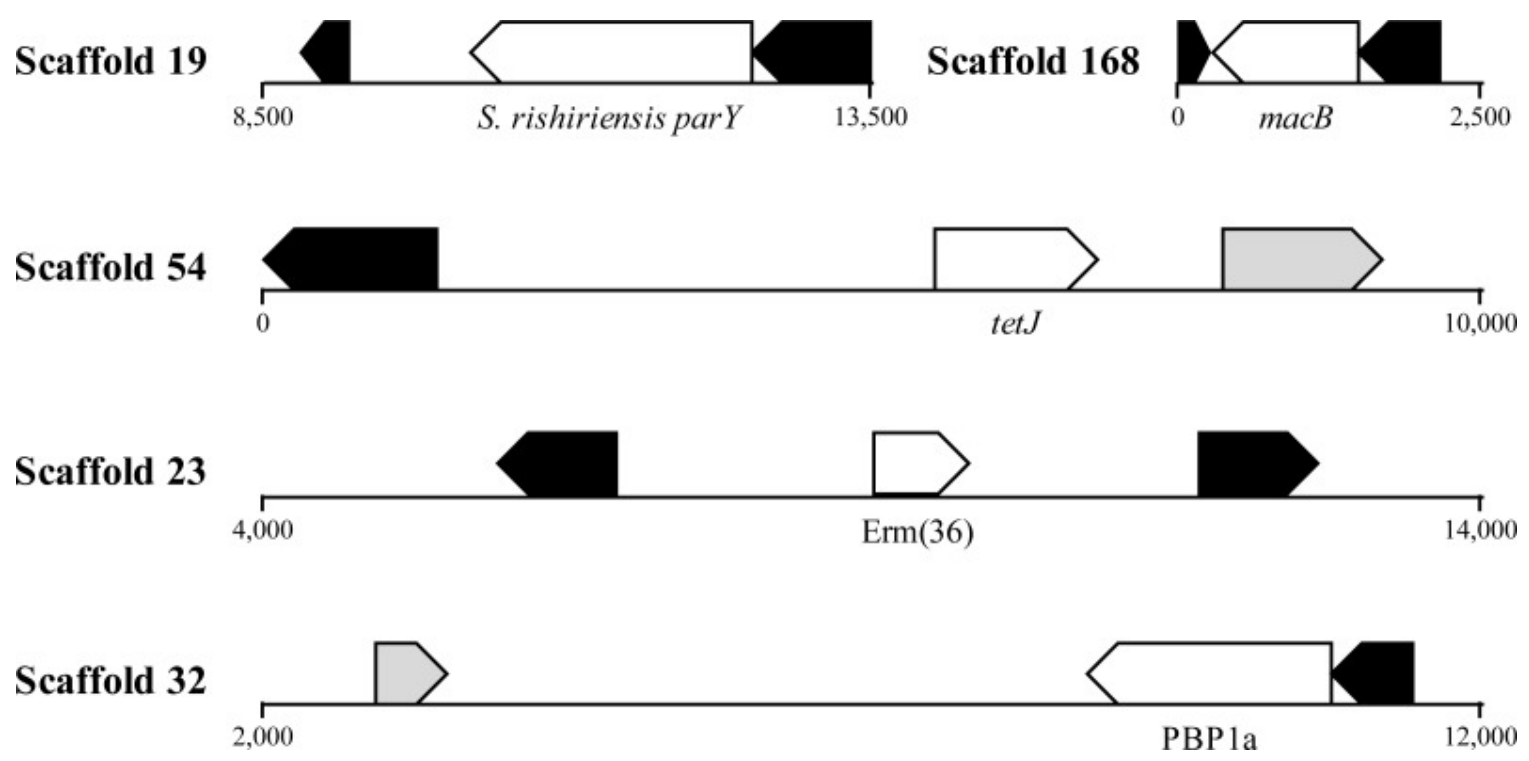

Figure D.4 - Location of ARGs indicates horizontal acquisition. Several antibiotic resistance genes (white) in A. cryaerophilus that are flanked by prophage genes (black) and transposases (grey) are shown. Nucleotide positions within each contig are also provided. 


\section{Presence and expression of virulence factors in sewage}

In addition to ARGs, another class of genes in A. cryaerophilus that could potentially impact human health is virulence factors. Most of the previous work at the molecular level has focused on nine putative virulence genes first described in Arcobacter butzleri strain RM4018. The presence of these nine virulence genes in Arcobacter genomes is highly variable and are all rarely found together in the same genome (Miller et al., 2007; Douidah et al., 2012). In general, the ability to adhere to and invade cells varies widely between Arcobacter species, with some of the most invasive strains isolated from feces or sewage samples (Ho et al., 2007; Karadas et al., 2013; Levican et al., 2013). Using the PATRIC database we identified 232 putative virulence genes (24 virulence categories) in A. cryaerophilus (Table S5, found in Additional_file_A14.pdf), out of which 231 were expressed (Additional_file_A18.csv). In PATRIC, virulence factors are assigned the category "virulence" if their mode of action is not specified in an associated study. Among the expressed virulence factor genes, 101 were annotated with a category other than "virulence." Of these, "intracellular survival and replication" was the largest group (30, 30\%) (Table S5, found in Additional_file_A14.pdf). The next largest groups present were "cellular metabolism" (22, 22\%), "adhesion" (18, 18\%), and "invasion" $(11,11 \%)$ (Figure D.5A). In the total sewage contigs, we identified 4,440 putative virulence factor genes (38 virulence categories (Table S6, found in Additional_file_A14.pdf), out of which, 3,776 were expressed (Additional_file_A18.csv)). Excluding the "virulence" category, 1,812 genes belonging to 37 other virulence categories were identified in the sewage microbiome. Of these, 1,589 genes from 35 categories were expressed, with 
(A)

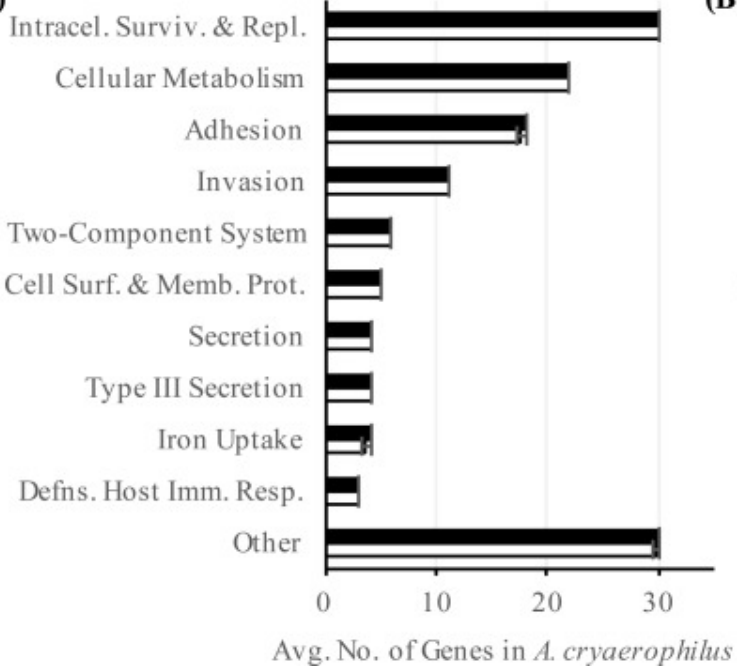

(B)

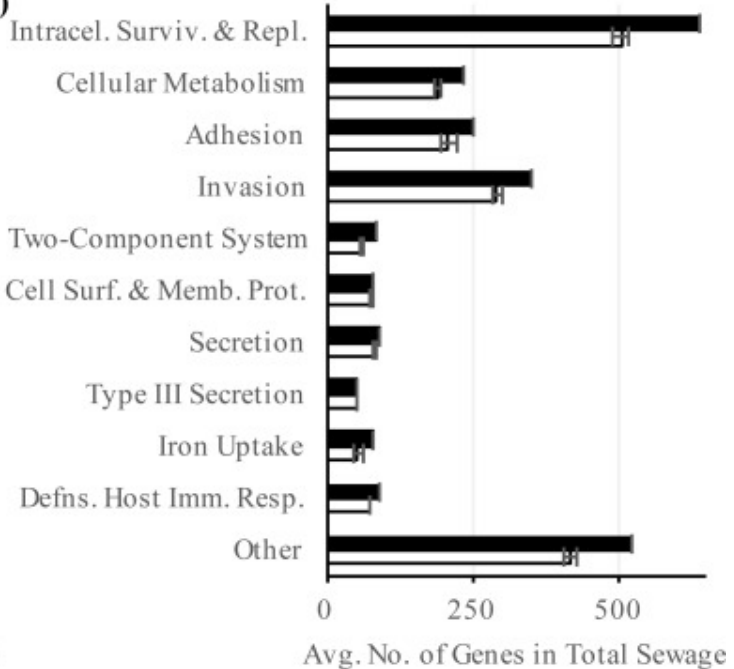

Figure D.5 - Abundance of virulence factors. Number of annotated (black) and expressed (white) virulence factor categories in (A) A. cryaerophilus and (B) total sewage sample is shown. Data represent means of three samples \pm standard deviations. 
"intracellular survival and replication" (548, 35\%), "invasion" (318, 20\%) and "adhesion" $(229,14 \%)$ being the top three categories (Figure D.5B; Table S6, found in

\section{Additional_file_A14.pdf).}

Our data suggest that untreated sewage contains several genes that potentially promote bacterial antibiotic resistance and virulence, and A. cryaerophilus, a potential human pathogen that contains multiple drug resistance and virulence factors, is a major component of this sewage system. Because we analyzed only a limited number of samples, further study is required to determine whether the dominance of $A$. cryaerophilus was a short-term phenomenon or whether this bacterium is a long-term resident of this sewage system (McLellan et al., 2010; Shanks et al., 2013). Although its cause is not understood, as observed in our study, Arcobacter has been shown to be highly prevalent in other sewage systems (Fisher et al., 2014). A possible explanation is the formation of biofilm on pipe surfaces and in deposited sediments along the sewer system (Chen, Leung \& Hung, 2003), another possibility is that the presence of multiple antibiotics, heavy metals or xenobiotics in wastewater, even at very low concentrations is selecting for A. cryaerophilus, which contains multiple ARGs (Heberer, 2002; Hawkey \& Jones, 2009; Gullberg et al., 2014; Jutkina et al., 2016). Similar to our observation, selection for antibiotic resistant bacteria has been described from other wastewater treatment plants (Goñi Urriza et al., 2000; Czekalski et al., 2012; Mao et al., 2015); consequently, constant monitoring of both pre- and post-treatment sewage is warranted because of the risk of reintroducing bacteria replete with ARGs and virulence factors into 
natural environments (Fahrenfeld et al., 2013; Czekalski, Gasco \& Burgmann, 2014; Mao et al., 2015; Pehrsson et al., 2016). 


\section{D.5 REFERENCES}

Abay S, Kayman T, Hizlisoy H, Aydin F. In vitro antibacterial susceptibility of Arcobacter butzleri isolated from different sources. Journal of Veterinary Medical Science. 2012;74:613-616.

Abdelbaqi K, Menard A, Prouzet-Mauleon V, Bringaud F, Lehours P, Megraud F. Nucleotide sequence of the gyrA gene of Arcobacter species and characterization of human ciprofloxacin-resistant clinical isolates. FEMS Immunology \& Medical Microbiology. 2007;49:337-345.

Albertsen M, Hugenholtz P, Skarshewski A, Nielsen KL, Tyson GW, Nielsen PH. Genome sequences of rare, uncultured bacteria obtained by differential coverage binning of multiple metagenomes. Nature Biotechnology. 2013;31:533-538.

Amos GCA, Zhang L, Hawkey PM, Gaze WH, Wellington EM. Functional metagenomic analysis reveals rivers are a reservoir for diverse antibiotic resistance genes. Veterinary Microbiology. 2014;171:441-447.

Arizona Department of Water Resources (ADWR) Arizona's water supplies and water demands. 2009. http://www.azwater.gov/AzDWR/PublicInformationOfficer/ documents/supplydemand.pdf

Baquero F, Martínez J-L, Cantón R. Antibiotics and antibiotic resistance in water environments. Current Opinion in Biotechnology. 2008;19:260-265.

Boetzer M, Henkel CV, Jansen HJ, Butler D, Pirovano W. Scaffolding pre-assembled contigs using SSPACE. Bioinformatics. 2011;27:578-579. 
Bolger AM, Lohse M, Usadel B. Trimmomatic: a flexible trimmer for Illumina sequence data. Bioinformatics. 2014;30:2114-2120.

Chen GH, Leung DHW, Hung JC. Biofilm in the sediment phase of a sanitary gravity sewer. Water Research. 2003;37:2784-2788.

Cho I, Blaser MJ. The human microbiome: at the interface of health and disease. Nature Reviews Genetics. 2012;13:260-270.

Collado L, Inza I, Guarro J, Figueras MJ. Presence of Arcobacter spp. in environmental waters correlates with high levels of fecal pollution. Environmental Microbiology. 2008;10:1635-1640.

Czekalski N, Berthold T, Caucci S, Egli A, Bürgmann H. Increased levels of multiresistant bacteria and resistance genes after wastewater treatment and their dissemination into lake geneva, Switzerland. Frontiers in Microbiology. 2012; 3:106.

Czekalski N, Gasco E, Burgmann H. Wastewater as a point source of antibioticresistance genes in the sediment of a freshwater lake. International Society for Microbial Ecology Journal. 2014;8:1381-1390.

Darriba D, Taboada GL, Doallo R, Posada D. jModelTest 2: more models, new heuristics and parallel computing. Nature Methods. 2012;9:772.

Douidah L, De Zutter L, Baré J, De Vos P, Vandamme P, Vandenberg O, Van den Abeele AM, Houf K. Occurrence of putative virulence genes in arcobacter species isolated from humans and animals. Journal of Clinical Microbiology. 2012; $50: 735-741$. 
Fahrenfeld N, Ma Y, Brien MO, Pruden A. Reclaimed water as a reservoir of antibiotic resistance genes: distribution system and irrigation implications. Frontiers in Microbiology. 2013;4:130.

Finn RD, Clements J, Eddy SR. HMMER web server: interactive sequence similarity searching. Nucleic Acids Research. 2011;39:W29-W37.

Fisher JC, Levican A, Figueras MJ, McLellan SL. Population dynamics and ecology of Arcobacter in sewage. Frontiers in Microbiology. 2014;5:525.

Gaze WH, Krone SM, Joakim Larsson DG, Li XZ, Robinson JA, Simonet P, Smalla K, Timinouni M, Topp E, Wellington EM, Wright GD, Zhu YG. Influence of humans on evolution and mobilization of environmental antibiotic resistome. Emerging Infectious Diseases. 2013;19:e120871.

Goñi Urriza M, Pineau L, Capdepuy M, Roques C, Caumette P, Quentin C. Antimicrobial resistance of mesophilic Aeromonas spp. isolated from two European rivers. Journal of Antimicrobial Chemotherapy. 2000;46:297-301.

Gullberg E, Albrecht LM, Karlsson C, Gullberg E, Albrecht LM, Karlsson C, Sandegren L, Andersson DI. Selection of a multidrug resistance plasmid by sublethal levels of antibiotics and heavy metals. mBio. 2014;5:e01918-14.

Hawkey PM, Jones AM. The changing epidemiology of resistance. Journal of Antimicrobial Chemotherapy. 2009;64:i3-i10.

Heberer T. Occurrence, fate, and removal of pharmaceutical residues in the aquatic environment: a review of recent research data. Toxicology Letters. 2002;131:517. 
Ho HTK, Lipman LJA, Gaastra W. Arcobacter, what is known and unknown about a potential foodborne zoonotic agent! Veterinary Microbiology. 2006;115:1-13.

Ho H.T, Lipman L.J, Hendriks H.G, Tooten P.C, Ultee T, Gaastra W. Interaction of Arcobacter spp. with human and porcine intestinal epithelial cells. FEMS Immunology \& Medical Microbiology. 2007;50:51-58.

Houf K, Devriese LA, Haesebrouck F, Vandenberg O, Butzler J-P, Van Hoof J, Vandamme P. Antimicrobial susceptibility patterns of Arcobacter butzleri and Arcobacter cryaerophilus strains isolated from humans and broilers. Microbial Drug Resistance. 2004;10:243-247.

Huelsenbeck JP, Ronquist F. MRBAYES: bayesian inference of phylogenetic trees. Bioinformatics. 2001;17:754-755.

Huson DH, Auch AF, Qi J, Schuster SC. MEGAN analysis of metagenomic data. Genome Research. 2007;17:377-386.

Hyatt D, LoCascio PF, Hauser LJ, Uberbacher EC. Gene and translation initiation site prediction in metagenomic sequences. Bioinformatics. 2012;28:2223-2230.

Jandhyala SA, Talukdar R, Subramanyam C, Vuyyuru H, Sasikala M, Reddy DN. Role of the normal gut microbiota. World Journal of Gastroenterology. 2015;21:87878803.

Jutkina J, Rutgersson C, Flach CF, Larsson DG. An assay for determining minimal concentrations of antibiotics that drive horizontal transfer of resistance. Science of the Total Environment. 2016;548-549:131-138. 
Kabeya H, Maruyama S, Morita Y, Ohsuga T, Ozawa S, Kobayashi Y, Abe M, Katsube Y, Mikami T. Prevalence of Arcobacter species in retail meats and antimicrobial susceptibility of the isolates in Japan. International Journal of Food Microbiology. 2004;90:303-308.

Karadas G, Sharbati S, Hänel I, Messelhäußer U, Glocker E, Alter T, Gölz G. Presence of virulence genes, adhesion and invasion of Arcobacter butzleri. Journal of Applied Microbiology. 2013;115:583-590.

Kearse M, Moir R, Wilson A, Stones-Havas S, Cheung M, Sturrock S, Buxton S, Cooper A, Markowitz S, Duran C, Thierer T, Ashton B, Meintjes P, Drummond A. Geneious Basic: an integrated and extendable desktop software platform for the organization and analysis of sequence data. Bioinformatics. 2012;28:1647-1649.

Krzywinski M, Schein J, Birol I, Connors J, Gascoyne R, Horsman D, Jones SJ, Marra MA. Circos: an information aesthetic for comparative genomics. Genome Research. 2009;19:1639-1645.

Langmead B, Salzberg SL. Fast gapped-read alignment with Bowtie 2. Nature Methods. 2012;9:357-359.

Levican A, Alkeskas A, Günter C, Forsythe SJ, Figueras MJ. Adherence to and invasion of human intestinal cells by Arcobacter species and their virulence genotypes. Applied and Environmental Microbiology. 2013;79:4951-4957.

Mao D, Yu S, Rysz M, Luo Y, Yang F, Li F, Hou J, Mu Q, Alvarez PJJ. Prevalence and proliferation of antibiotic resistance genes in two municipal wastewater treatment plants. Water Research. 2015;85:458-466. 
McArthur AG, Waglechner N, Nizam F, Yan A, Azad MA, Baylay AJ, Bhullar K, Canova MJ, De Pascale G, Ejim L, Kalan L, King AM, Koteva K, Morar M, Mulvey MR, O’Brien JS, Pawlowski AC, Piddock LJV, Spanogiannopoulos P, Sutherl AD, Tang I, Taylor PL, Thaker M, Wang W, Yan M, Yu T, Wright GD. The comprehensive antibiotic resistance database. Antimicrobial Agents and Chemotherapy. 2013;57:3348-3357.

McLellan SL, Huse SM, Mueller-Spitz SR, Andreihcheva EN, Sogin ML. Diversity and population structure of sewage derived microorganisms in wastewater treatment plant influent. Environmental Microbiology. 2010;12:378-392.

Merga JY, Williams NJ, Miller WG, Leatherbarrow AJH, Bennett M, Hall N, Ashelford KE, Winstanley C. Exploring the diversity of Arcobacter butzleri from cattle in the UK using MLST and whole genome sequencing. PLOS ONE. 2013;8(2): e55240.

Miller WG, Parker CT, Rubenfield M, Mendz GL, Wösten MMSM, Ussery DW, Stolz JF, Binnewies TT, Hallin PF, Wang G, Malek JA, Rogosin A, Stanker LH, Mandrell RE. The complete genome sequence and analysis of the Epsilonproteobacterium Arcobacter butzleri. PLOS ONE. 2007;2(12):e1358.

Mitchell A, Chang H-Y, Daugherty L, Fraser M, Hunter S, Lopez R, McAnulla C, McMenamin C, Nuka G, Pesseat S, Sangrador-Vegas A, Scheremetjew M, Rato C, Yong S-Y, Bateman A, Punta M, Attwood TK, Sigrist CJA, Redaschi N, Rivoire C, Xenarios I, Kahn D, Guyot D, Bork P, Letunic I, Gough J, Oates M, Haft D, Huang H, Natale DA, Wu CH, Orengo C, Sillitoe I, Mi H, Thomas PD, 
Finn RD. The InterPro protein families database: the classification resource after 15 years. Nucleic Acids Research. 2015;43:D213-D221.

Morita Y, Maruyama S, Kabeya H, Boonmar S, Nimsuphan B, Nagai A, Kozawa K, Nakajima T, Mikami T, Kimura H. Isolation and phylogenetic analysis of Arcobacter spp. in ground chicken meat and environmental water in Japan and Thailand. Microbiology and Immunology. 2004;48:527-533.

Noyes NR, Yang X, Linke LM, Magnuson RJ, Cook SR, Zaheer R, Yang H, Woerner DR, Geornaras I, McArt JA, Gow SP, Ruiz J, Jones KL, Boucher CA, McAllister TA, Belk KE, Morley PS. Characterization of the resistome in manure, soil and wastewater from dairy and beef production systems. Scientific Reports. 2016; 6:24645.

Okoh AI, Odjadjare EE, Igbinosa EO, Osode AN. Wastewater treatment plants as a source of microbial pathogens in receiving watersheds. Journal of Biotechnology. 2007;6:2932-2944.

Overbeek R, Olson R, Pusch GD, Olsen GJ, Davis JJ, Disz T, Edwards RA, Gerdes S, Parrello B, Shukla M, Vonstein V, Wattam AR, Xia F, Stevens R. The SEED and the Rapid Annotation of microbial genomes using Subsystems Technology (RAST) Nucleic Acids Research. 2014;42:D206-D214.

Paulsen IT, Sliwinski MK, Saier MH. Microbial genome analyses: global comparisons of transport capabilities based on phylogenies, bioenergetics and substrate specificities. Journal of Molecular Biology. 1998;277:573-592. 
Pehrsson EC, Tsukayama P, Patel S, Mejía-Bautista M, Sosa-Soto G, Navarrete KM, Calderon M, Cabrera L, Hoyos-Arango W, Bertoli MT, Berg DE, Gilman RH, Dantas G. Interconnected microbiomes and resistomes in low-income human habitats. Nature. 2016;533:212-216.

Peng Y, Leung HCM, Yiu SM, Chin FYL. IDBA-UD: A de novo assembler for singlecell and metagenomic sequencing data with highly uneven depth. Bioinformatics. 2012;28:1420-1428.

Perner M, Gonnella G, Kurtz S, LaRoche J. Handling temperature bursts reaching 464C: different microbial strategies in the sisters peak hydrothermal chimney. Applied and Environmental Microbiology. 2014;80:4585-4598.

Pima Association of Governments (PAG) Existing wastewater treatment facilities and other point source NPDES discharges. 2006. https:/www.pagnet.org/documents/ Water/PC208/Ch5_Apr06.pdf

Poole K. Bacterial stress responses as determinants of antimicrobial resistance. Journal of Antimicrobial Chemotherapy. 2012;67:2069-2089.

Rahimi E. Prevalence and antimicrobial resistance of Arcobacter species isolated from poultry meat in Iran. British Poultry Science. 2014;55:174-180.

Rizzo L, Manaia C, Merlin C, Schwartz T, Dagot C, Ploy MC, Michael I, Fatta-Kassinos D. Urban wastewater treatment plants as hotspots for antibiotic resistant bacteria and genes spread into the environment: a review. Science of the Total Environment. 2013;447:345-360. 
Schroeder-Tucker L, Wesley IV, Kiehlbauch JA, Larson DJ, Thomas LA, Erickson GA. Phenotypic and ribosomal RNA characterization of Arcobacter species isolated from porcine aborted fetuses. Journal of Veterinary Diagnostic Investigation. 1996;8:186-195.

Shanks OC, Newton RJ, Kelty CA, Huse SM, Sogin ML, McLellan SL. Comparison of the microbial community structures of untreated wastewaters from different geographic locales. Applied and Environmental Microbiology. 2013;79:29062913.

Sievers F, Wilm A, Dineen D, Gibson TJ, Karplus K, Li W, Lopez R, McWilliam H, Remmert M, Soding J, Thompson JD, Higgins DG. Fast, scalable generation of high-quality protein multiple sequence alignments using Clustal Omega. Molecular Systems Biology. 2011;7:539.

Stamatakis A, Hoover P, Rougemont J. A rapid bootstrap algorithm for the RAxML web servers. Systematic Biology. 2008;57:758-771.

Talavera G, Castresana J. Improvement of phylogenies after removing divergent and ambiguously aligned blocks from protein sequence alignments. Systematic Biology. 2007;56:564-577.

Udikovic-Kolic N, Wichmann F, Broderick NA, Handelsman J. Bloom of resident antibiotic-resistant bacteria in soil following manure fertilization. Proceedings of the National Academy of Sciences. 2014;111:15202-15207. 
Vandenberg O, Dediste A, Houf K, Ibekwem S, Souayah H, Cadranel S, Douat N, Zissis G, Butzler JP, Vandamme P. Arcobacter species in humans. Emerging Infectious Diseases. 2004;10:1863-1867.

Varela AR, Manaia CM. Human health implications of clinically relevant bacteria in wastewater habitats. Environmental Science and Pollution Research. 2013; $20: 3550-3569$.

Versluis D, D’Andrea MM, Ramiro Garcia J, Leimena MM, Hugenholtz F, Zhang J, Öztürk B, Nylund L, Sipkema D, Van Schaik W, De Vos WM, Kleerebezem M, Smidt H, Van Passel MWJ. Mining microbial metatranscriptomes for expression of antibiotic resistance genes under natural conditions. Scientific Reports. 2015; $5: 11981$.

Wattam AR, Abraham D, Dalay O, Disz TL, Driscoll T, Gabbard JL, Gillespie JJ, Gough R, Hix D, Kenyon R, Machi D, Mao C, Nordberg EK, Olson R, Overbeek R, Pusch GD, Shukla M, Schulman J, Stevens RL, Sullivan DE, Vonstein V, Warren A, Will R, Wilson MJC, Yoo HS, Zhang C, Zhang Y, Sobral BW. PATRIC, the bacterial bioinformatics database and analysis resource. Nucleic Acids Research. 2014;42:D581-D591.

Wellington EMH, Boxall ABA, Cross P, Feil EJ, Gaze WH, Hawkey PM, JohnsonRollings AS, Jones DL, Lee NM, Otten W, Thomas CM, Williams AP. The role of the natural environment in the emergence of antibiotic resistance in Gramnegative bacteria. The Lancet Infectious Diseases. 2013;13:155-165. 
Zhang T, Shao M-F, Ye L. 454 Pyrosequencing reveals bacterial diversity of activated sludge from 14 sewage treatment plants. The ISME Journal. 2012;6:1137-1147.

Zhou Y, Liang Y, Lynch KH, Dennis JJ, Wishart DS. PHAST: a fast phage search tool. Nucleic Acids Research. 2011;39:W347-W352.

Zhu Q, Kosoy M, Dittmar K. HGTector: An automated method facilitating genome-wide discovery of putative horizontal gene transfers. BMC Genomics. 2014;15:717. 


\section{APPENDIX E}

Whole-genome sequence of Coxiella burnetii Nine Mile RSA 439 (phase II, clone 4), a laboratory workhorse strain

\section{E.1 ABSTRACT}

Here, we report the whole-genome sequence of Coxiella burnetii Nine Mile RSA439

(phase II, clone 4), a laboratory strain used extensively to investigate the biology of this intracellular bacterial pathogen. The genome consists of a 1.97-Mb chromosome and a 37.32-kb plasmid. 


\section{E.2 GENOME ANNOUNCEMENT}

Coxiella burnetii is a Gram-negative intracellular bacterium that causes an influenza-like illness in humans called Q fever (Eldin et al. 2017). Most infections occur through inhalation of aerosols originating from domestic livestock operations. Within the host cell, the pathogen becomes metabolically activated upon delivery into an acidic lysosome-like vacuole (Voth and Heizen 2007). The only C. burnetii virulence factor established in an immunocompetent animal model of infection is full-length lipopolysaccharide (LPS), which is synthesized by virulent phase I bacteria (Moos and Hackstadt 1987). Upon serial in vitro passage, phase I bacteria convert to avirulent phase II bacteria, which produce truncated LPS lacking O antigen and several core sugars (Amano and Williams 1984; Hackstadt 1986; Fiset 1957; Hackstadt 1990; Kersh et al. 2011).

The Nine Mile RSA439 (phase II, clone 4) strain (NMII) was derived from the Nine Mile strain, which was originally isolated in 1935 from the tick Dermacentor andersoni in Montana (Davis and Cox 1938). The Nine Mile strain was passaged 94 times in embryonated hen's eggs and then plaque purified to generate NMII (Wike et al. 1972; Williams et al. 1981). NMII has an $\sim 26-\mathrm{kb}$ chromosomal deletion that eliminates several LPS biosynthetic genes and is associated with the production of a severely truncated LPS (Hackstadt et al. 1985; Vodkin and Williams 1986; Hoover et al. 2002; Beare et al. 2006; Denison et al. 2007). Because of clonality, avirulence in a guinea pig model of infection, and lack of phase reversion, NMII is considered a biosafety level 2 
(BSL-2) bacterium (Moos and Hackstadt 1987; Hackstadt 1996; Samuel and Hendrix 2009). Other C. burnetii strains are considered BSL-3 bacteria and are regulated as select agents by the U.S. Centers for Disease Control and Prevention (Samuel and Hendrix 2009).

The NMII genome has not yet been sequenced; consequently, most researchers use the published genome of the Nine Mile RSA493 phase I strain (NMI) for reference (Seshadri et al. 2003). This occasionally leads to inconclusive results; for instance, the gene caeA is not annotated as a functional protein-coding gene in the NMI genome, but transcriptome analysis of NMII indicates its presence (Bisle et al. 2016; Raghavan 2016). Thus, a fully annotated genome of the widely used NMII laboratory strain is needed to better understand the unique biology of this intracellular pathogen.

NMII was grown in ACCM-2 at $37^{\circ} \mathrm{C}$ in a $2.5 \% \mathrm{O}_{2} / 5 \% \mathrm{CO}_{2}$ environment in a trigas incubator (New Brunswick Scientific, NJ), as described previously (Omsland et al. 2011). DNA was isolated from a 500-ml 7-day culture using phenol-chloroform with gentle cell disruption using a vortex adapter (Qiagen, CA) in order to minimize DNA fragmentation. DNA was sequenced using the PacBio RS II platform (Pacific Biosciences, USA), which generated a library containing 86,731 reads with an average length of 7,565 bp. Reads were assembled using HGAP 2.3.0 (Chin et al. 2013), which returned three contigs. The two chromosome contigs and the plasmid contig were closed in SSPACE 2.0 (Boetzer et al. 2011) using trimmed Illumina MiSeq 75-bp paired-end reads (5.50 million). Finally, CLC Genomics Workbench 6.5 (Qiagen) was used to map all Illumina and PacBio reads to the NMII chromosome $(410 \times$ and $290 \times$ coverage, 
respectively) and plasmid (240× and $30 \times$ coverage, respectively) scaffolds to generate the consensus genome sequence. As expected, relative to the genome of NMI, homologs of genes CBU_0679 to CBU_0697 were completely deleted from the NMII genome (Moos and Hackstadt 1987; Vodkin and Williams 1986; Hoover et al. 2002; Beare et al. 2006; Denison et al. 2007). In addition, partial deletions of homologs of CBU_0678, CBU_0698, and CBU_0918 and several single nucleotide polymorphisms were observed.

\section{Accession numbers}

The complete genome sequence of C. burnetii Nine Mile RSA439 (phase II, clone 4) has been deposited in GenBank under the accession numbers CP020616 (for chromosome) and CP020617 (for plasmid). 


\section{E.3 REFERENCES}

Amano K, Williams JC. Chemical and immunological characterization of lipopolysaccharides from phase I and phase II Coxiella burnetii. J Bacteriol. 1984;160:994-1002.

Beare PA, Samuel JE, Howe D, Virtaneva K, Porcella SF, Heinzen RA. Genetic diversity of the $\mathrm{Q}$ fever agent, Coxiella burnetii, assessed by microarray-based wholegenome comparisons. J Bacteriol. 2006;188:2309-2324. doi:

10.1128/JB.188.7.2309-2324.2006.

Bisle S, Klingenbeck L, Borges V, Sobotta K, Schulze-Luehrmann J, Menge C, Heydel C, Gomes JP, Lührmann A. The inhibition of the apoptosis pathway by the Coxiella burnetii effector protein CaeA requires the EK repetition motif, but is independent of survivin. Virulence. 2016;7:400-412. doi:

10.1080/21505594.2016.1139280.

Boetzer M, Henkel CV, Jansen HJ, Butler D, Pirovano W. Scaffolding pre-assembled contigs using SSPACE. Bioinformatics. 2011;27:578-579. doi: 10.1093/bioinformatics/btq683.

Chin CS, Alexander DH, Marks P, Klammer AA, Drake J, Heiner C, Clum A, Copeland A, Huddleston J, Eichler EE, Turner SW, Korlach J. Nonhybrid, finished microbial genome assemblies from long-read SMRT sequencing data. Nat Methods. 2013;10:563-569. doi: 10.1038/nmeth.2474. 
Davis GE, Cox HR. A filter-passing infectious agent isolated from ticks. I. Isolation from Dermacentor andersonii, reactions with animals, and filtration experiments. Publ Health Rep. 1938;53:2259-2276. doi: 10.2307/4582746.

Denison AM, Massung RF, Thompson HA. Analysis of the O-antigen biosynthesis regions of phase II isolates of Coxiella burnetii. FEMS Microbiol Lett. 2007;267:102-107. doi: 10.1111/j.1574-6968.2006.00544.x.

Eldin C, Mélenotte C, Mediannikov O, Ghigo E, Million M, Edouard S, Mege JL, Maurin M, Raoult D. From Q fever to Coxiella burnetii infection: a paradigm change. Clin Microbiol Rev. 2017;30:115-190. doi: 10.1128/CMR.00045-16.

Fiset P . Phase variation of Rickettsia (Coxiella) burneti; study of the antibody response in guinea pigs and rabbits. Can J Microbiol. 1957;3:435-445. doi: 10.1139/m57046.

Hackstadt T. Antigenic variation in the phase I lipopolysaccharide of Coxiella burnetii isolates. Infect Immun. 1986;52:337-340.

Hackstadt T. The role of lipopolysaccharides in the virulence of Coxiella burnetii. Ann N Y Acad Sci. 1990;590:27-32. doi: 10.1111/j.1749-6632.1990.tb42203.x.

Hackstadt T. Biosafety concerns and Coxiella burnetii. Trends Microbiol. 1996;4:341342. doi: 10.1016/0966-842X(96)81555-1.

Hackstadt T, Peacock MG, Hitchcock PJ, Cole RL. Lipopolysaccharide variation in Coxiella burnetii: intrastrain heterogeneity in structure and antigenicity. Infect Immun. 1985;48:359-365. 
Hoover TA, Culp DW, Vodkin MH, Williams JC, Thompson HA. Chromosomal DNA deletions explain phenotypic characteristics of two antigenic variants, phase II and RSA 514 (crazy), of the Coxiella burnetii nine mile strain. Infect Immun. 2002;70:6726-6733. doi: 10.1128/IAI.70.12.6726-2733.2002.

Kersh GJ, Oliver LD, Self JS, Fitzpatrick KA, Massung RF . Virulence of pathogenic Coxiella burnetii strains after growth in the absence of host cells. Vector Borne Zoonotic Dis. 2011;11:1433-1438. doi: 10.1089/vbz.2011.0670.

Moos A, Hackstadt T. Comparative virulence of intra- and interstrain lipopolysaccharide variants of Coxiella burnetii in the guinea pig model. Infect Immun. 1987;55:1144-1150.

Omsland A, Beare PA, Hill J, Cockrell DC, Howe D, Hansen B, Samuel JE, Heinzen RA. Isolation from animal tissue and genetic transformation of Coxiella burnetii are facilitated by an improved axenic growth medium. Appl Environ Microbiol. 2011;77:3720-3725. doi: 10.1128/AEM.02826-10.

Raghavan R. A repeat motif on a Coxiella effector protein facilitates apoptosis inhibition. Virulence. 2016;7:369-371. doi: 10.1080/21505594.2016.1156834.

Samuel JE, Hendrix LR. Laboratory maintenance of Coxiella burnetii. Curr Protoc Microbiol. 2009; Chapter 6:Unit 6C.1. doi: 10.1002/9780471729259.mc06c01s15. Seshadri R, Paulsen IT, Eisen JA, Read TD, Nelson KE, Nelson WC, Ward NL, Tettelin H, Davidsen TM, Beanan MJ, Deboy RT, Daugherty SC, Brinkac LM, Madupu R, Dodson RJ, Khouri HM, Lee KH, Carty HA, Scanlan D, Heinzen RA, Thompson HA, Samuel JE, Fraser CM, Heidelberg JF. Complete genome 
sequence of the Q-fever pathogen Coxiella burnetii. Proc Natl Acad Sci.

2003;100:5455-5460. doi: 10.1073/pnas.0931379100.

Vodkin MH, Williams JC. Overlapping deletion in two spontaneous phase variants of Coxiella burnetii. J Gen Microbiol. 1986;132:2587-2594. doi: $10.1099 / 00221287-132-9-2587$.

Voth DE, Heinzen RA. Lounging in a lysosome: the intracellular lifestyle of Coxiella burnetii. Cell Microbiol. 2007;9:829-840. doi: 10.1111/j.14625822.2007.00901.x.

Wike DA, Tallent G, Peacock MG, Ormsbee RA. Studies of the rickettsial plaque assay technique. Infect Immun. 1972;5:715-722.

Williams JC, Peacock MG, McCaul TF. Immunological and biological characterization of Coxiella burnetii, phases I and II, separated from host components. Infect Immun. 1981;32:840-851. 